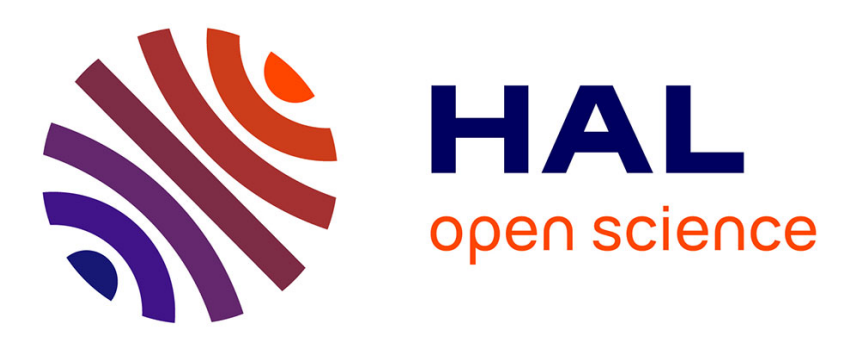

\title{
A review of microstructural changes occurring during FSW in aluminium alloys and their modelling
}

\author{
Dimitri Jacquin, Gildas Guillemot
}

\section{To cite this version:}

Dimitri Jacquin, Gildas Guillemot. A review of microstructural changes occurring during FSW in aluminium alloys and their modelling. Journal of Materials Processing Technology, 2021, 288, pp.116706. 10.1016/j.jmatprotec.2020.116706 . hal-02911059

\section{HAL Id: hal-02911059 \\ https://hal.science/hal-02911059}

Submitted on 3 Aug 2020

HAL is a multi-disciplinary open access archive for the deposit and dissemination of scientific research documents, whether they are published or not. The documents may come from teaching and research institutions in France or abroad, or from public or private research centers.
L'archive ouverte pluridisciplinaire HAL, est destinée au dépôt et à la diffusion de documents scientifiques de niveau recherche, publiés ou non, émanant des établissements d'enseignement et de recherche français ou étrangers, des laboratoires publics ou privés. 


\title{
A review of microstructural changes occurring during FSW in aluminium alloys and their modelling
}

\author{
Dimitri Jacquin ${ }^{\text {a) }}$ Gildas Guillemot ${ }^{\text {b) }}$ \\ a) University of Bordeaux, I2M CNRS, Site IUT, 15, rue Naudet - CS 10207, \\ 33175 Gradignan Cedex, France \\ b) MINES ParisTech, PSL Research University, CEMEF UMR CNRS 7635, \\ CS10207, 06904 Sophia Antipolis, France
}

\begin{abstract}
:
Friction stir welding (FSW) process is currently considered as a promising alternative to join aluminium alloys. Indeed, this solid-state welding technique is particularly recommended for the assembly of these materials. Since parts are not heated above their melting temperature, FSW process may prevent solidification defects encountered in joining aluminium alloys and known as limitations to the dissemination of these materials in industries. During the past years, large literature has been devoted to the modelling of microstructural evolution in aluminium alloys during FSW processes and mainly dedicated to the analysis of precipitate evolutions and grain recrystallization mechanisms. Precipitate size distribution models have aroused widespread interest in recent years demonstrating their relevance to follow precipitation process in multicomponent alloys and multiphase systems. Efficient recrystallization models are also available and based on various grain growth mechanisms. In addition, multi-scale coupling strategies have recently emerged considering thermal, mechanical and metallurgical solutions. Consequently, the effect of FSW process parameters on weld properties is now investigated to determine optimized welding strategies regarding microstructure evolution. This research is based on reliable models reported in the literature enhancing the estimation of final weld state and associated properties as an answer to industrial needs. Validations of proposed modelling strategies have been reported based on in-depth analyses of experimental observations. This present work proposes a review of recent models dedicated to microstructural evolutions in aluminium alloys during FSW process. The interest and efficiency of current approaches will be discussed to highlight their limitations. Guidelines will propose new routes toward enhanced modelling strategies for future developments.
\end{abstract}

\section{Keywords:}

Friction Stir Welding; Aluminium alloys; Microstructure modelling; Precipitation process; Recrystallization mechanism; Guidelines 


\section{Contents}

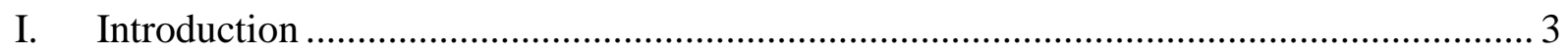

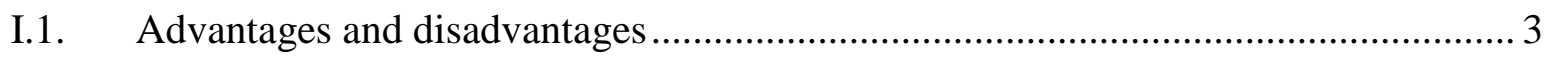

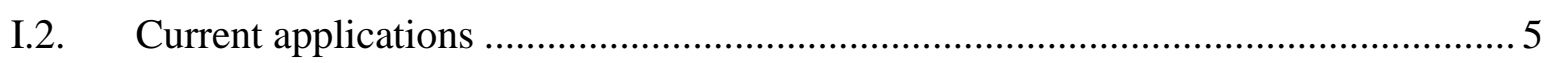

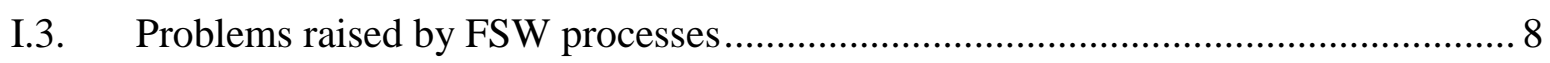

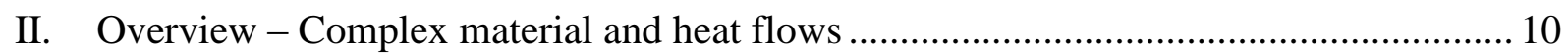

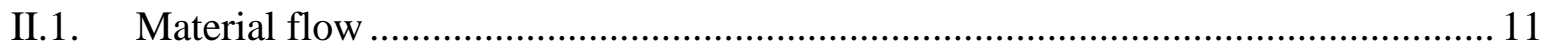

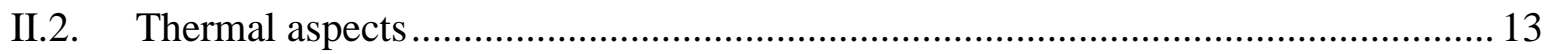

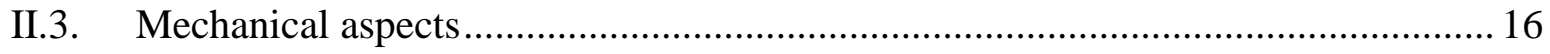

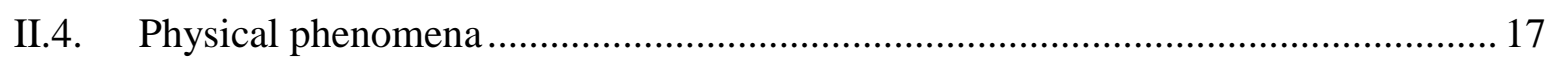

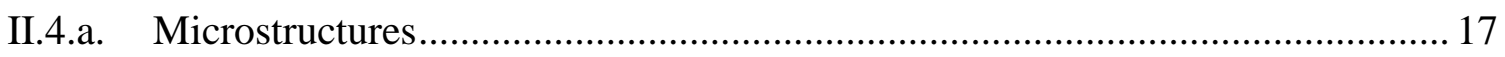

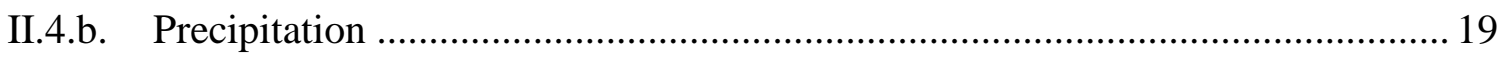

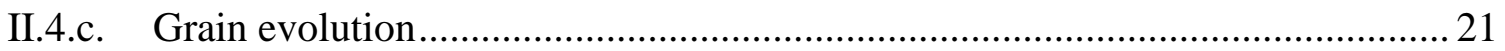

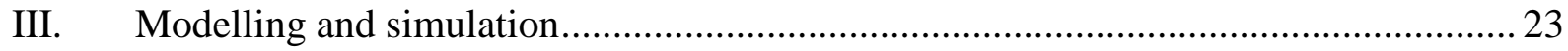

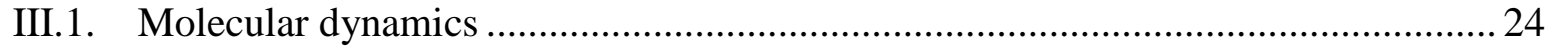

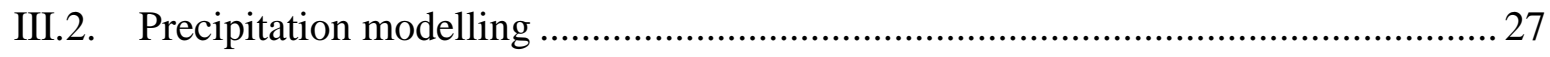

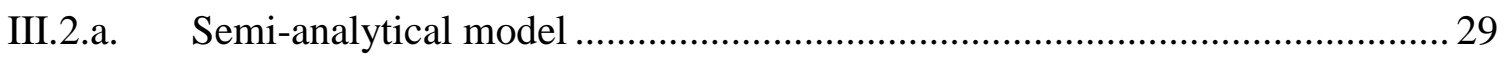

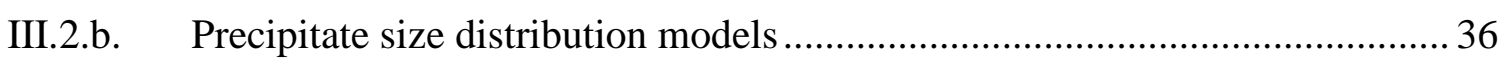

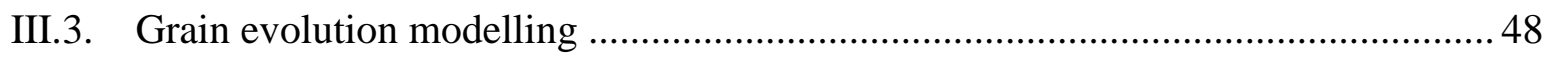

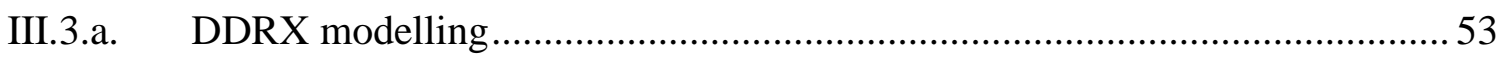

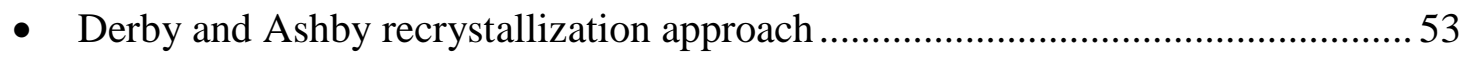

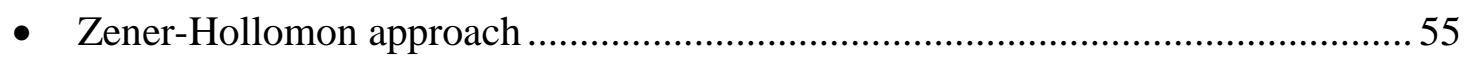

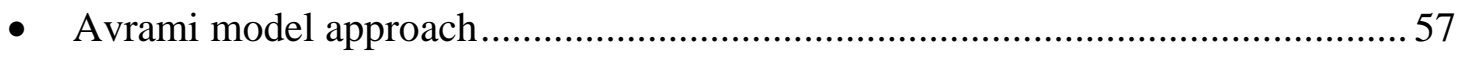

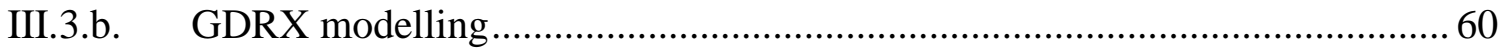

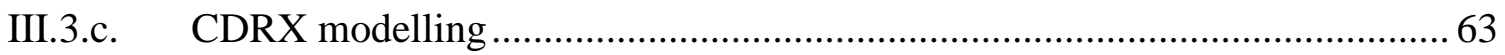

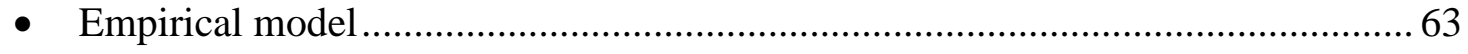

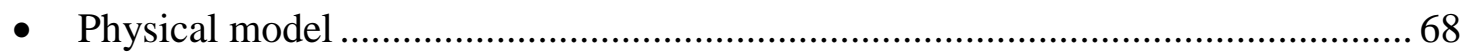

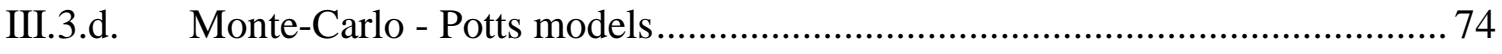

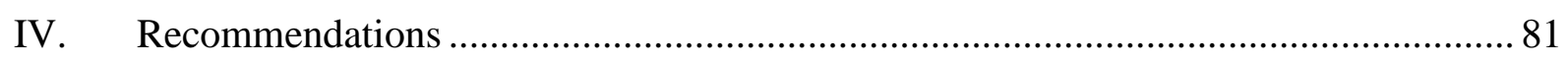

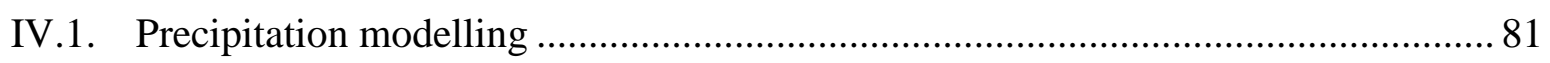

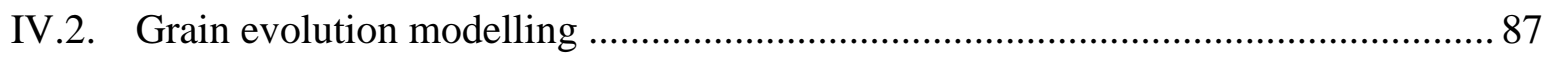

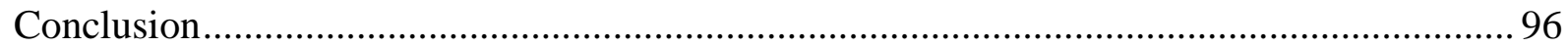

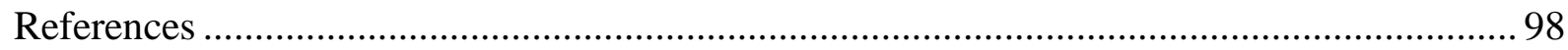




\section{Introduction}

Since its discovery in 1991 by Thomas (1991) at The Welding Institute (TWI, 2019), Friction stir welding (FSW) process has become a technique of choice in the joining of aluminium components. Wang et al. (2008) have demonstrated that this process is able to produce thick assemblies with both high mechanical properties and large fatigue performances. Microstructures observations show fine grains and restricted Heat Affected Zone limiting cracks development. Sahu and Pal (2017) obtained similar results when considering welding of aluminium alloys with dissimilar thickness and various joining configurations. Tensile and yield strength measured after joining processes were found close to the base material properties. Mechanical performances are usually better in FSW processes than those obtained by conventional joining processes as fusion welding also considering lower level of residual stresses. This process is developed in order to increase local temperature by plastic deformation to achieve stirring domain. A rigid cylindrical tool consisting of a threaded pin and a shoulder rotates and slowly plunges into the junction line between two parts placed end to end. Friction and stirring generate heat and soften the material, allowing local plastic deformation and material mixing. Process control prevents melting of parts during joining process thus restricting material transformations to solid state.

In a process of lightening aeronautical structures, welding of aluminium alloys offers an alternative to traditional bolting or riveting processes, allowing to overcome the major drawbacks of these techniques: heterogeneous junction, mass contribution by added metal, stress concentration close to the holes decreasing fatigue resistance. Consequently FSW process results in a weight saving as well as a reduction in the manufacturing costs. These advantages are clearly attractive to answer current industrial needs. Consequently, the use of FSW processes in industries is a major economic and technical challenge for the aircraft, shipbuilding or even automotive industries where first applications have recently emerged. In this context, FSW is also a particularly promising process for future years.

\section{I.1. Advantages and disadvantages}

Wang et al. (2008) consider that the greatest advantage of FSW process lies in the possibility to weld so-called "non-weldable" aluminium alloys grades by conventional methods (arc welding, laser welding). As an example, Kalemba and Dymek (2016) have developed a thorough analysis on microstructure evolution in AA7136 aluminium alloy (Al-Zn-Mg-Cu) during FSW process. The authors justified their interest by the restricted range of industrial applications for theses alloys due to the difficulties encountered in their welding by conventional methods. FSW process is therefore of great interest in promoting mechanical properties and microstructure required by industries. More generally, Blondeau (2013) highlight the possibility to apply FSW on certain alloys of the 2000 and 7000 series usually non suitable for joining processes based on fusion welding. Indeed, these alloys are characterized by a structural hardening and thus the transition to the liquid state and the 
sudden cooling, as in the case of laser welding process, causes loss in hardness. Moreover, these alloys may have very large solidification intervals which make them sensitive to hot cracking. Finally, as noticed by TWI (2019), the high temperatures involved in fusion welding processes cause burning phenomena, especially in the case of aluminium grades alloyed with copper (2000 series).

Friction Stir Welding is a thermomechanical process affecting the material located under and around the friction head. The heat generated during the welding causes a phase change in the solid state, leading to high temperatures allowing the material to reach the hot deformation regime in the vicinity of the friction head. It should be noticed that heat is mainly induced by the tool shoulder rather than the pin. The temperatures usually do not exceed $500{ }^{\circ} \mathrm{C}$, which corresponds to approximately $75 \%$ of the melting temperature in industrial aluminium alloys. In this temperature range, aluminium alloys usually behave as thermoviscous materials. The use of lower temperature gradients compared with conventional welding processes limits the occurrence of hot cracking and final deformations as shown on pieces presented in Fig. 1. Similarly, Ma et al. (2013) demonstrated that the assemblies made in FSW on aluminiumlithium alloys (AA2198 aluminium grades) have a very good mechanical strength. On average, this latter is equal to $79 \%$ of the one associated to the base material even if a decrease in mechanical properties is observed when input energy increases. Lomolino et al. (2005) also discussed on the fatigue resistance observed in friction stir welded components and their enhancement compared to conventional welding processes. A relevant overview on fatigue properties of welded pieces in also provided considering literature data and design data. In addition, as a mechanical process, FSW is easy to automate. Moreover, this process does not require consumables such as filler metal or protective gas for the weld beads. Research projects developed in past years have resulted in a large application of this reduced cost process in a broad range of industrial sectors and on materials other than aluminium alloys, also including dissimilar welds.

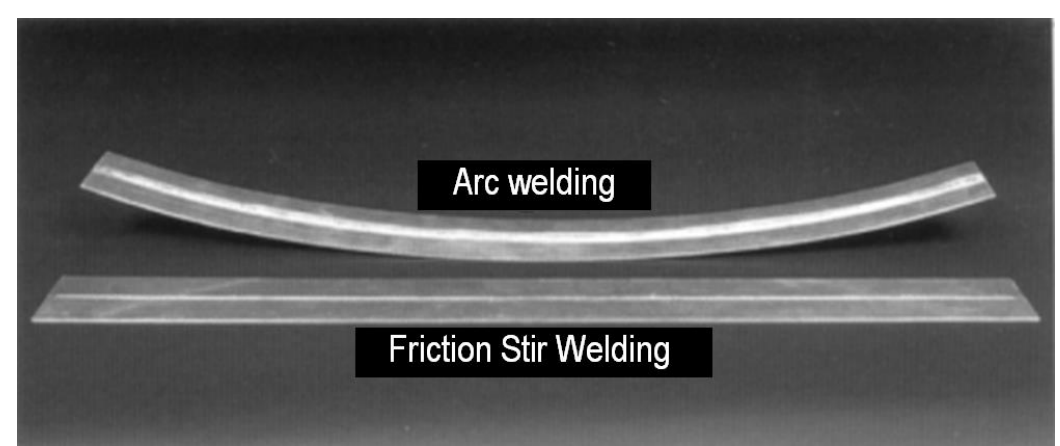

Fig. 1: Comparison of the distortion caused by FSW and by arc welding on aluminium sheets of $5 \mathrm{~mm}$ thickness (Cazes (2003)).

VIA Inno (2017) shows that Friction Stir Welding is a materials joining technique attracting genuine interest from industrial players with nearly 4400 patent families registered over the last 10 years. This activity of patent filing has intensified over the past years, marked by an increase of almost $70 \%$ between 2005 and 2018 of patents associated to Friction Stir Welding 
processes (Fig. 2 b) according to Scopus (2019). Beyond the interest of industrial players, the scientific community is also increasingly interested in this process, as evidenced by the scientific production which has grown by a factor 4 on the same period (Fig. 2 a).

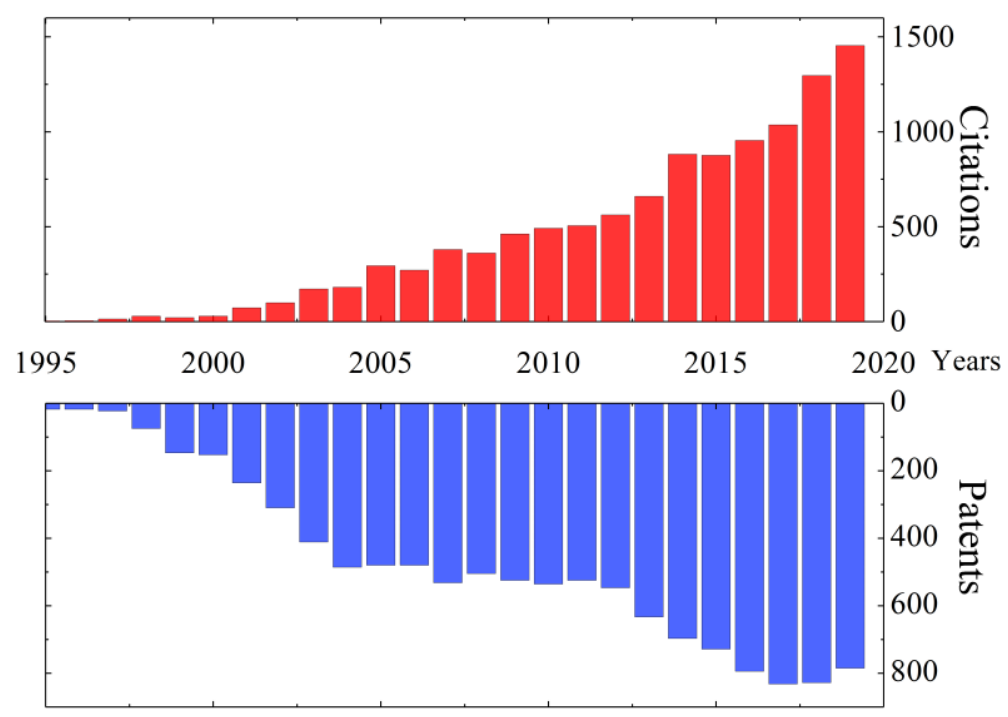

Fig. 2: Evolution of worldwide article citations and patent applications related to Friction Stir Welding process since 1995 (Scopus, 2019).

Nowadays, FSW is a well mastered and reliable technology. This joining process has been integrated in many manufacturing methods in various industrial sectors. FSW processes have advantages compared to conventional welding processes as detailed by TWI (2019). The level of defects is decreased as melting is prevented. Solidification defects such as hot cracking, liquation cracking, weld porosities or segregation phenomena does not occur consequently. These differences are well discussed by Kah et al. (2015) in an overview of weld defects occurring on aluminium alloys when comparing FSW and fusion welding processes. It should also be mentioned that the aesthetics of welds is improved compared to fusion welding technologies. In addition, the initial investment is quite low and the operating costs are competitive, also considering the quality/price ratio. Moreover, efficiency of FSW processes is also observed considering energy consumption.

\section{I.2. Current applications}

FSW has found a wide range of applications. Since the invention of FSW process, research has made it possible to use this process in many structures, mainly in the transport industry. Gibson et al. (2014) show that the repeatability and reliability of the process combined with its ability to assemble light alloys attract aerospace applications. More especially, Rambabu et al. (2017) mentioned that the materials most commonly used in the aerospace field are aluminium alloys of the 2000 and 7000 series. As an example, the alloys of the 7000 series are used as stiffeners for 2000 series overlay panels as highlighted by Legrand et al. (2015b). Heinz et al. (2000) have demonstrated that the use of aluminium alloys is still maintained 
thanks to the ongoing improvements made in their production by industries. As long as these alloys are used in aerospace industry, the FSW will continue to arouse the interest of industry. Indeed, applications dedicated to these grades produce high quality welds compared to other welding techniques also considering the high level of quality required in this sector. In particular, FSW is increasingly used as a replacement for riveting in aerospace structures. In the aerospace industry, FSW technologies have been widely adopted by many companies, such as Boeing, Lockheed Martin or Marshall Space Flight Center. Since 1999, Boeing Space has launched Delta flares whose fuel tanks are welded by FSW process as a substitute of the more expensive TIG process. NASA was particularly interested in FSW process for its Spacecraft Orion (Fig. 3 a) where the FSW, contrarily to the other conventional welding methods, allowed creating high strength ultra-lightweight aluminium alloy welds required to withstand the harsh environments encountered during a space flight. In aeronautics, the main improvements are expected in future applications for the welding of hull stiffeners in place of riveting (fuselages, wings, cryogenic tanks, airplane tanks and releasable tanks, rockets).

The FSW process has also been utilized extensively in the construction of marine vessels. To date, several applications are in the industrial production stage. The first application concerns light alloy panels for refrigeration installations on fishing boats, made by the juxtaposition of extruded profiles joined by FSW process. In these large-scale manufactures, the resulting small distortions are of particular interest for shipping companies. Hovercraft and cruise ships are also built from lightweight prefabricated modules. As an example, the Super Liner Ogasawara (Mitsui Engineering and Shipbuilding, Japan) (Fig. 3 b) is reported as the largest ship manufactured with FSW processes. This advantage associated together with productivity benefit, has supported generally the decision to use FSW processes as joining technology in shipbuilding. Consequently, FSW process has also changed profoundly the way high-speed ferries are manufactured.

(a)

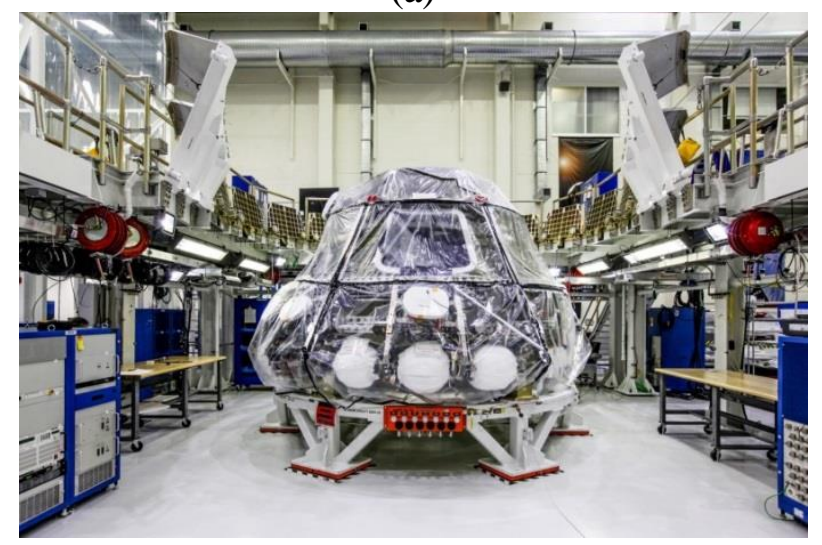

(b)

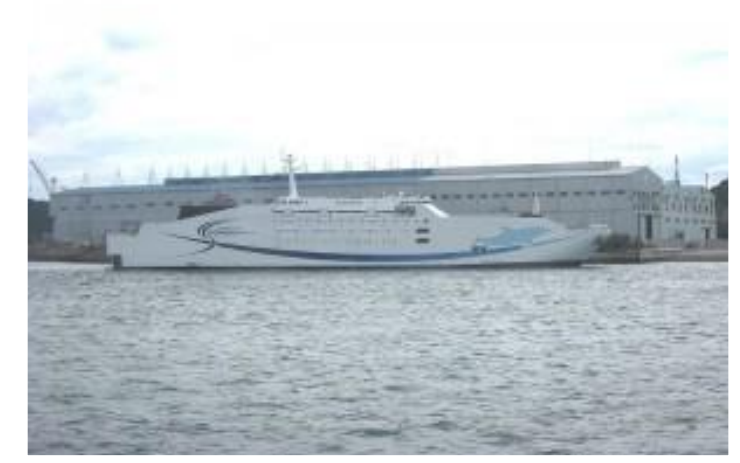

Fig. 3: Current constructions developed with use of FSW processes: (a) Nasa's Orion Spacecraft (NASA, 2019) (b) The Super Liner Ogasawara (CSFP, 2019) 
The large use of aluminium alloys of the 6000 series for the manufacture of bodyworks in the automobile and railway industry promotes also the development of FSW processes in this sector since these aluminium grades have few limitations to hot forming. As an example, FSW is used in the worldwide production of railway carriages. European manufacturers (ALSTOM, Siemens) and Japanese (Hitachi, Kawasaki, Sumitomo, Showa, Nippon Light Metals) operate on industrial scale for the construction of high-speed trains (floors, pavilions, body sides). Despite their initial hesitations about this new process, industrials funded research projects that currently diversify FSW applications without limitation to transport industry. For instance, FSW is used to seamlessly join aluminium surfaces of the next generation of Apple iMac computers. Today, numerous tests are in progress to confirm the reliability of the process. In addition, it should be pointed out that TWI (2019) continues to develop FSW technologies in order to diffuse and disseminate this process in industries. The range of materials and joint configurations has been extended and weld properties have been enhanced. Consequently, FSW is now easier to adopt for industries. Several innovative approaches related to FSW have also recently emerged as detailed hereafter with their main characteristics in Table 1:

Table 1: Recent innovative industrial processes related to Friction Stir Welding (TWI, 2019).

\begin{tabular}{ll}
\hline \hline \multicolumn{1}{c}{ Name } & \multicolumn{1}{c}{ Characteristic } \\
\hline \hline AdStir & Use of a filler metal during welding to add material \\
\hline Corner FSW & Developed T-sections and corner welds based on stationary shoulder \\
\hline Floating-Bobbin FSW & $\begin{array}{l}\text { Welding technics without backing plate - Probe and shoulder are free } \\
\text { to float in the tool holder }\end{array}$ \\
\hline Robotic FSW & $\begin{array}{l}\text { Use of a robot instead of a conventional rigid machine to follow } \\
\text { three-dimensional joint lines and enhance flexibility }\end{array}$ \\
\hline Stationary Shoulder & $\begin{array}{l}\text { The probe rotates and protrudes through a hole in a stationary } \\
\text { shoulder/slide component to limit heat introduction at surface }\end{array}$ \\
\hline Thick-Section FSW & $\begin{array}{l}\text { Twin-sided welding to offer the potential to weld materials up to 150 } \\
\text { mm thick in a single pass }\end{array}$ \\
\hline Thin-Section FSW & $\begin{array}{l}\text { Joining material sheets as thin as 0.3 mm thick using stationary } \\
\text { shoulder with smooth external surface and minimal weld undercut }\end{array}$ \\
\hline \hline
\end{tabular}

These recent processes derivate from FSW and are based on the experience gained by industries. They demonstrate the innovation and dynamism in joining processes based on the mixing of metallic material. All these processes lead to large evolutions in microstructure of joined material and may be worthy of investigation. However, this paper will only focus on the classical FSW processes. Nevertheless the analysis proposed hereafter would be easily extended to others FSW configurations. 


\section{I.3. Problems raised by FSW processes}

FSW process induces microstructural changes, residual stresses and distortions sometimes difficult to control and monitor for manufacturers. All these phenomena can be responsible for a deterioration of end-use properties, in terms of geometry, assembly quality, mechanical strength and fatigue resistance. In an increasingly competitive industrial environment, companies are constrained to develop their products on time and at lower costs. The control of manufacturing processes and associated properties on finished products may be considered as an essential success factor. In this context, the characterization and numerical modelling of FSW processes are of particular interest to investigate the feasibility of assembling pieces, to optimize the operating parameters or to analyse the in-service strength. A relevant example is the development of the Trivex tool designed by the TWI. Colegrove and Shercliff (2004a) have developed a detailed modelling of the two-dimensional flow evolution around this tool during joining process aiming to optimize the forces exerted during the welding phase. Colegrove and Shercliff (2004b) have also simultaneously proposed an extension to three-dimensional flow based on similar research activities. Nandan et al. (2008) have also highlighted that controlling FSW process implies to master complex interactions between various thermomechanical phenomena. Indeed, this process involves a large range of interdependent relationships, at a smaller scale, between the microstructure and thermal and mechanical aspects as plastic deformation, dynamic recrystallization or heating and cooling rate. All these complex interactions have large consequences on the mechanical integrity of welding and its in-service performance.

More recently, Agelet De Saracibar (2019) has developed an in-depth analysis of the challenges associated to the numerical modelling of FSW processes showing the difficulties associated to this task. Indeed, FSW involves non-linear physical coupled phenomena at various spatial and time scales. As precisely detailed by Agelet De Saracibar, several complex issues have to be tackled in order to face the problems associated to the development of relevant models able to simulate FSW processes as an answer to industrial needs. Three very strongly coupled aspects emerge whose interactions alone constitute the essential part of the complexities of material evolution modelling in FSW processes:

- Thermal phenomena, reflected in temperature rise and cooling during welding

- Metallurgical evolutions, related to phase transformations, grain size and texture of the material

- Mechanical phenomena, inherent to the friction, the strain and the stresses induced during welding. 
The interdependencies of these three quantities are shown schematically in Fig. 4 based on the classical interdependence between thermal, mechanical and metallurgical process evolution.

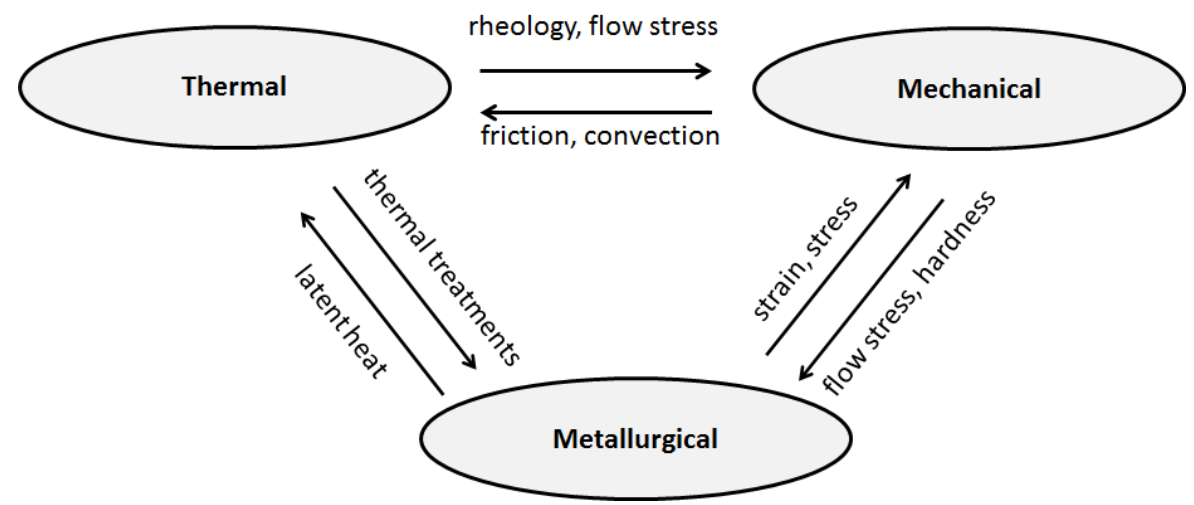

Fig. 4: Interaction diagram showing the physical phenomena involved during the FSW process and their interactions.

Several objectives can be addressed in microstructure evolution modelling during FSW process. Indeed, microstructural modelling can be used to create specifically tailored material microstructures with optimized properties as well as residual stress states enhancing components performances. This advance leads also to the development of production equipment based on FSW as better suited joining technology with reduced premature wear and increased service life. This latter process reduces production time and thereby associated costs. The influence of the material structure evolution on mechanical behaviour can also be considered in order to enhance final properties. This approach is however not yet common practice in industry, where yield loci and strain hardening curves are usually the sole considered data describing mechanical behaviour. The influence of the material's structure, such as grain size and grain texture characteristics, is rarely taken into account by multiparameter yield loci. Indeed, experiments to characterize such models can be complex as tests are not always standardized and need to be repeated when another material structural state is considered. In addition, the influence of stress or temperature fields on microstructure evolution has also to be considered in such analysis. However, the ability to simulate the manufacture of metal products and their performance in service has increased tremendously over the past two decades. The spectacular developments in computer performance have made possible to build much larger and more accurate CAE (Computer-Aided Engineering) numerical tools. Materials can also be addressed on progressively smaller scales, taking into account the role of the material structure on macroscopic mechanical behaviour. In this approach, the development of small scale or in-situ observations as SAXS (Small-Angle Xray Scattering) experiments also correspond to new opportunities in FSW research. Steuwer et al. (2011) were the first to develop a wide range of in-depth complementary technics including SAXS observations to provide a detailed description of microstructure observed in AA2199-Li Alloy after FSW process. De Geuser et al. (2014) also developed SAXS observations however dedicated to AA2050 Al-Li-Cu aluminium alloy in the T8 state including heat treatments to mimic temperature evolution during process. Such observations 
give the possibility to map microstructure evolution in FSW processes and offer a better understanding of physical phenomena inducing phase change, precipitation processes or grain structure evolution. These observations are also supplemented with micro-hardness mapping providing direct comparisons between precipitations processes and mechanical properties.

Material models that are able to directly capture the microstructure evolution and its influence on mechanical behaviour are thus in great demand. Indeed, they can cover a wide range of process conditions and reduce the characterization effort. As an example the European project ENABLE (2019) aims at developing tailored material microstructures with improved properties and performances through new solutions proposed to master FSW processes.

In the next future, CAE tools dedicated to the modelling of microstructure evolution in FSW process and its consequence should be developed as an answer to industry's needs. Indeed, simulation tools are currently seldom available when considering FSW process. Research activities currently developed in Abaqus (2019) or Sysweld (2019) only correspond to first developments and are mainly restricted to thermo-mechanical evolution of materials. The economic benefits of approaches dedicated to material evolution during FSW process may be significant, regarding the cost of the experimental tests and the effort required identifying model parameters often leading to tedious iterative steps. New perspectives in terms of processing conditions and weight reduction can also be thought when offering better capabilities to predict microstructure evolution and its effect during extreme solicitations. CAE vendors able to deliver such material approaches for industrial simulations would have strong competitive advantages, especially regarding sheet metal forming, machining, additive manufacturing and welding processes. The aim of this paper is to review hereafter the methods developed in recent years to model microstructure evolutions induced by FSW process on aluminium alloys and its consequence on materials properties. The first part will provide an overview of mechanical, thermal and metallurgical phenomena involved during FSW process. In addition, equipment, tools and methodologies developed to follow material evolution will be described. The second part will present recent progress reported in literature on modelling of microstructural evolution in FSW mainly considering precipitation and recrystallization phenomena. The last part will focus on perspectives in these research fields for future activities. More specifically, some advices, suggestions and proposals of guidelines will be depicted in order to improve the knowledge on material evolution at the microstructure scale.

\section{Overview - Complex material and heat flows}

Microstructure resulting from FSW is due to the large rate of deformation occurring during process. The friction, the large stresses and strains encountered by the material induce a complex thermal history. Many authors have focused on the modelling of heat and material flux in order to predict the evolution of the microstructure and the properties of the welded joint. 


\section{II.1. Material flow}

The plastic deformation generated by the rotational and linear movements of the tool has been the subject of several sometimes-contradictory interpretations. These studies allow us, inter alia, to visualize the trajectories of material elements. The first studies were those of Li et al. (1999) who used two grades of aluminium (2024 and 6061) to visualize the mixture of material between the two sheets using the contrast difference obtained in optical microscopy by appropriate chemical polishing. This technique gives an indication of the final material mixture but does not allow determining the precise movement of material during welding (Fig. 5). Seidel and Reynolds (2001) follow the transfer of matter at different locations in the thickness by inserting 5454 aluminium alloy markers into two 2195 alloy sheets to weld. They notice a difference in the flow between the advancing side and the retreating side.

(a)

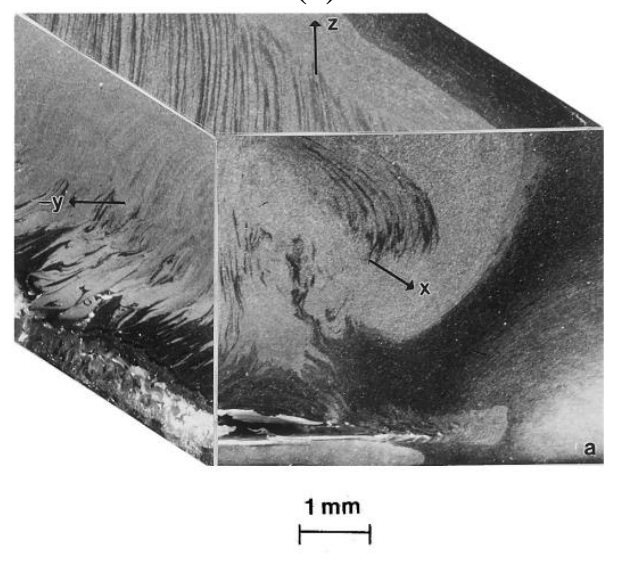

(b)

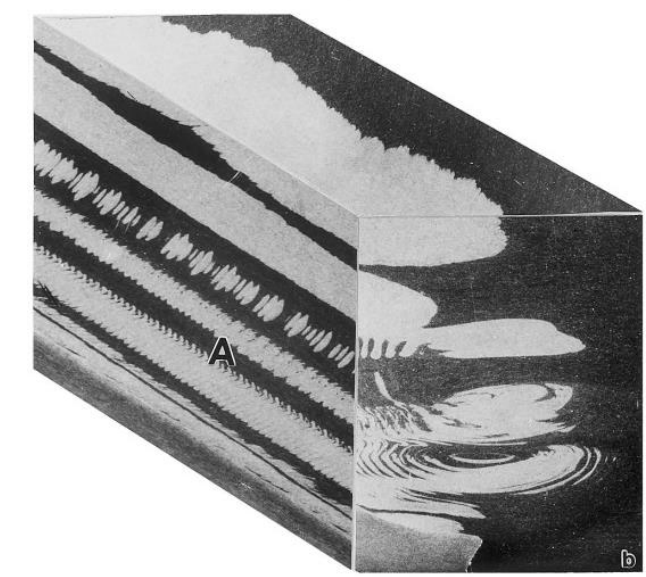

Fig. 5: Visualization of material flows through the welded domain between aluminium sheet (AA2024 (white) and AA6061 (black)) developed for two tool orientations: (a) / normal direction and (b) canted with $\sim 2^{\circ}$ (Li et al., 1999).

Colligan (1999) uses small steel beads (easily detected by radiography), inserted into the welding plates, as markers. Using this technique, the authors observe that the material above the sheets is driven counterclockwise on the retreating side while undergoing a slight rise in front of the tool before being drained downwards and dispersed behind the tool at a depth slightly greater than their initial depth. The material located slightly deeper is also driven in the same direction although rising continuously from front to back without being scattered behind the tool. The material at the bottom of the tool is extruded under the pin and the tool rotation has a slight influence on its evolution. Schmidt et al. (2006) use an innovative technique known as the "stop-action" method. This approach consists in stopping instantaneously the advance movement of the tool and in simultaneously engaging its withdrawal of the material without affecting the shape printed in the material by the tool. 
(a)

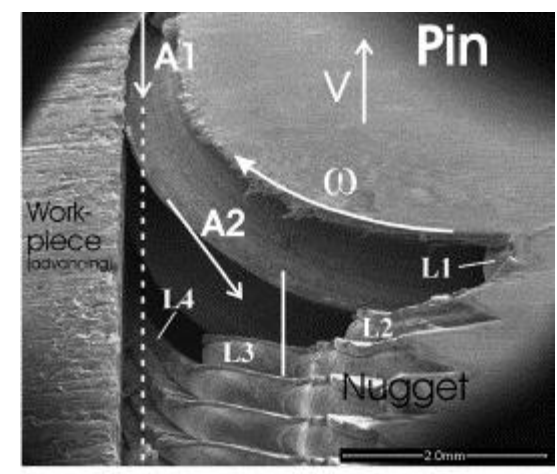

(b)

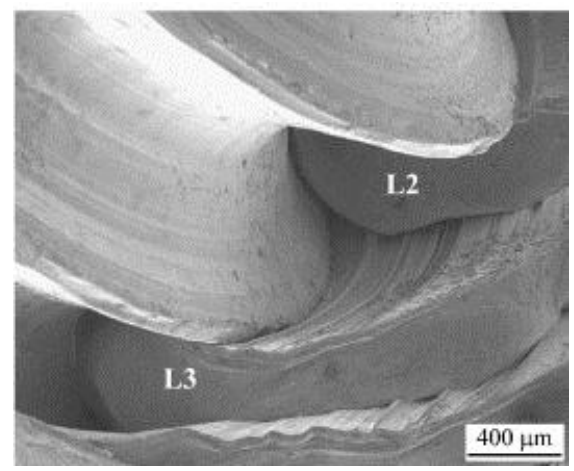

Fig. 6: SEM images of a pin-workpiece couple sampled from a weld made with $v=5.2 \mathrm{~mm} \mathrm{~s}^{-1}, \omega=710 \mathrm{rpm}$ (thus $\lambda=440 \mu \mathrm{m}$ ). (a) A low magnification view: (b) A higher magnification view from the direction as indicated by A2 in (a) (Chen and Cui, 2008).

Chen and Cui (2008) developed observations (Fig. 6) showing a typical result of these imprints by optical microscopy but leaving the tool in the weld. They perform micrographs of the piece immersed in the material so as to observe how the pin interacts with the layer of sheared material in its proximity and propose an explanation on the origin of concentric bands observed in the core of the weld named 'onion rings'. The feature of these 'onion rings' is explained as a cutting effect of the semi-cylinder shape structures developed in the nugget. These structures are a consequence of the extrusion of metal around the retreating side of the tool as reported by Krishnan (2002). Their spacing is of the order of the advance during a tool rotation. However, the development of these structures is usually considered as having low impact on the final properties of the weld. Reynolds et al. (2000b) analysed the flow of aluminium-lithium alloy (AA2195-T8) in many FSW-welded joints using the flow visualisation technique. This technique consists in following the movement of markers inserted into the aluminium sheet in domains corresponding to the nugget and the thermomechanical affected zone. Observations are developed after etching of successive milled surfaces when difference in copper compositions between alloy and markers provides threedimensional information on markers final positions.

All these observations testify the huge deformation encountered by the material during the welding. Kumar et al. (2018) used particle image velocimetry (PIV) technique, to study the material flow and measure the strain rate around the tool (Fig. 7). Two-dimensional flow patterns were analysed using small glass balls tracers in a transparent material. The authors have carefully chosen materials with similar effective density so that the tracers do not alter material flow during the process. They showed that the material could rotate around the tool several times before its exit. This technique has also made possible the study of the shape and development of onion rings in welding. The authors showed that the particles velocities were higher in the retreating side than at advancing side at the same distance from the pin. The 
maximum strain rate at $170 \mathrm{rpm}$ and $50 \mathrm{~mm} \cdot \mathrm{min}^{-1}$ was estimated to $20 \mathrm{~s}^{-1}$. The material flow during FSW process is complex depending on the tool geometry, process parameters and welded materials. Therefore, any generalization of such estimation has to be analysed very carefully. In general, the measurement of thermomechanical fields in FSW is extremely complex as transformations take place inside the stir zone, which is subject to extreme strain, preventing the installation of any measuring devices.

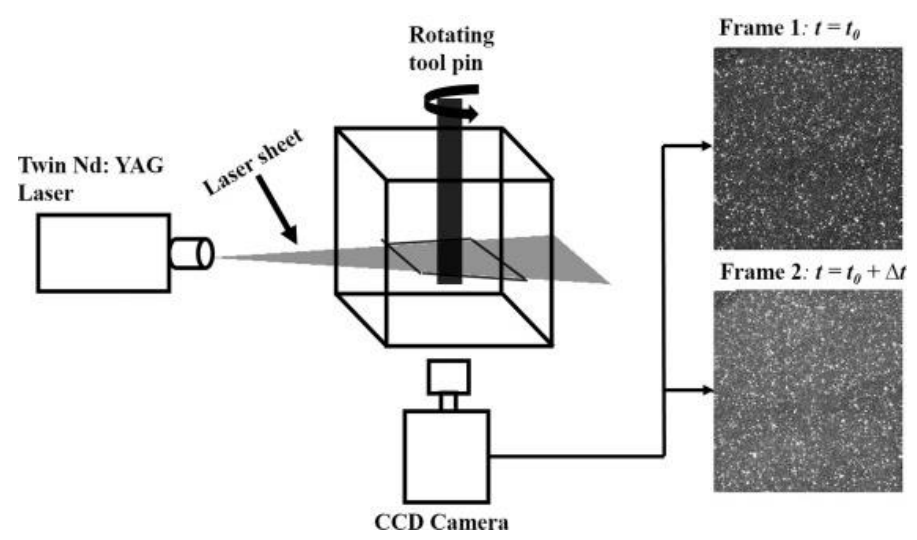

Fig. 7: Flow measurement using particle image velocimetry (PIV) system and images of the tracer particles for time ' $t=t_{0}$ ' and ' $t=t_{0}+\Delta t$ ' showing their displacements. (Kumar et al., 2018).

\section{II.2. Thermal aspects}

As mentioned previously, heat is mainly induced in the weld by the tool shoulder rather than the pin. Various partitions are provided in the literature in order to determine precisely the origins of heat introduced in materials during FSW process and the sources on the tool surface. According to Colegrove (2000), the heat source induced by pin rotation may exceed $20 \%$ of the total heat generation. Gallais et al. (2008) gave an estimation of $\sim 80 \%$ for the shoulder and $\sim 20 \%$ for the pin as heat source origins in their process modelling. Hattel et al. (2009) provide an estimation of $83 \%$ for the shoulder, $16 \%$ for the tool sides and only $1 \%$ for its tip, however considering a process efficiency of $88 \%$. According to Hofmann and Vecchio (2007) the model proposed by Schmidt et al. (2004) shows that only 14\% of the heat is generated by the tip of the bit and its surface. In the model derived by Song et al. (2003), this ratio decreases to only $2 \%$ for the bit. In addition, Hofmann and Vecchio provided a detailed description on the evolution of these ratios in Friction Stir Processing (FSP) and Submerged Friction Stir Processing (SFSP) depending from the tool geometry and based on their own modelling. Dual nature of heat generation has also been emphasized and discussed by Colligan and Mishra (2008). Indeed, heat is generated both by friction and plastic deformation considering the local evolution of materials, also depending on whether there is a local motion between tool and workpiece or a local seizure. No clear distinction is 
made possible experimentally in order to distinguish mechanisms. As a consequence numerical models usually include both origins in heat generation term.

The knowledge of the actual temperature in the welded zones (Heat Affected Zone - HAZ, Thermomechanically Affected Zone - TMAZ and Stir Zone - SZ) is important not only to determine the thermal contribution on the weld microstructure evolution but also for the thermal model calibration. Recording the temperature during the FSW process is tricky. The deformation generated by the process does not allow a continuous temperature measurement in the welded zones. Most authors instrument the welded plates with thermocouples providing access to the temperature evolution in the zones outside the tool path. Extrapolations are developed afterwards to estimate evolutions in the welded zone. More precisely, this approach was developed by Jacquin (2011) in order to investigate separately the tool rotation and welding velocity effects when applying FSW process on AA2024 aluminium grade. Li et al. (2012) also developed temperature measurement on AA2024 aluminium grade demonstrating that temperature evolutions when comparing advancing and retreating sides show nonsignificant differences in accordance with previous results reported in literature. Hattel et al. (2009) instrumented a weld experiment on 7075 T6 aluminium alloy leading to clear validation of simulated thermal evolutions compared to experimental observations after adjustment of heat transfer coefficient and contact resistances. In their opinion, the coupling between workpiece and backing plate is of prime importance. Silva et al. (2017) have also noticed the link between weld temperatures and weld quality showing the interest to control temperature evolution in order to master defects development during FSW. An overview has been proposed by these authors on temperature measurement solutions reported in literature. Three strategies are compared with i) thermocouples embedded in the tool, ii) thermocouples introduced in the workpiece and iii) the tool-workpiece thermocouple (TWT) strategy where temperature measurement is based on the thermoelectric effect developed between tool and workpiece. This latter device provides accurate and fast measurements and seems to have consequently clear advantages according to the authors. Recently, Silva-Magalhães et al. (2019) have applied and extended this approach in order to provide measurements in several locations around the tool by coupling TWT device with thermocouples inserted in the tool. This approach was successfully applied on thick AA6082-T6 aluminium alloys to provide temperature distribution. The method was also relevant to localise hottest and lowest temperature positions in the tool neighbourhood. Some authors as $\mathrm{Li}$ et al. (2006) place these thermocouples in the deformed zone to record the temperature till their destruction by the tool. Santiago et al. (2009) developed thermographic maps with infrared camera (Fluke Ti30) on AA6061-T6 aluminium alloy in order to extract temperature profile in front of the tool in the aim to validate simulations results. Uncertainties were lower than $2 \%$ according to Santiago. To determine the temperature at the shoulder of the tool, Lammlein et al. (2009) also used a thermal imaging camera. Using this tool, Bitondo et al. (2010) showed that the heat source reaches a steady state rapidly after the beginning of the process. Richards et al. (2006) have used the thermal camera for the calibration of heat sources and convective exchanges of their thermal model in a validation approach. However, thermal cameras are 
restricted to the temperature surface measurement. Serio et al. (2016) also investigated temperature field evolution using infrared camera (Canon EOS 40D) on AA5754-H111 aluminium alloys with the aim to correlate temperature changes with evolutions in mechanical properties and weld quality. According to Serio, this approach demonstrates the potentiality of thermography to monitor and control FSW processes on-line regarding specific data associated to measurements (heating slope, temperature profile). Nevertheless, Magalhães (2016) assumes that the repeatability associated to thermal camera measurements is compromised considering the environmental heat source as the hot tool. Moreover the large reflectivity associated to aluminium surfaces leads to some uncertainties. The observation domain is also limited by the shoulder preventing measurements in domains of highest temperatures.

Nevertheless, the means dedicated to temperature recording are not limited to thermocouples and thermal cameras. Indeed, high strain gradients are locally generated inducing heat fluxes whose knowledge is of interest for both computing and metallurgist engineers. It is therefore necessary to estimate precisely the temperature in the welded zone. In this case, Guerdoux (2007) used instrumented FSW tools with inserted thermocouples. Silva-Magalhães et al. (2019) reported several studies on such approach in their literature review and demonstrate the interest of this strategy. This method enables a continuous measurement of the temperature at the tool / material contact during the entire welding phase (plunging, feeding, and retraction). As a consequence, temperature evolution and its associated modelling can be achieved during the transient regimes. However, the thermal resistance of the tool / material interface should not be overlooked. In order to summarize all the knowledge on this subject, Colligan (2008) developed a conceptual model for the process variables related to heat generation in FWS on aluminium. The authors proposed clear and detailed mapping of all thermal interactions encountered in the process. Regarding heat generation, the authors place particular emphasis on the distinction between friction and plastic deformation effects (Fig. 8) as both mechanisms occur.

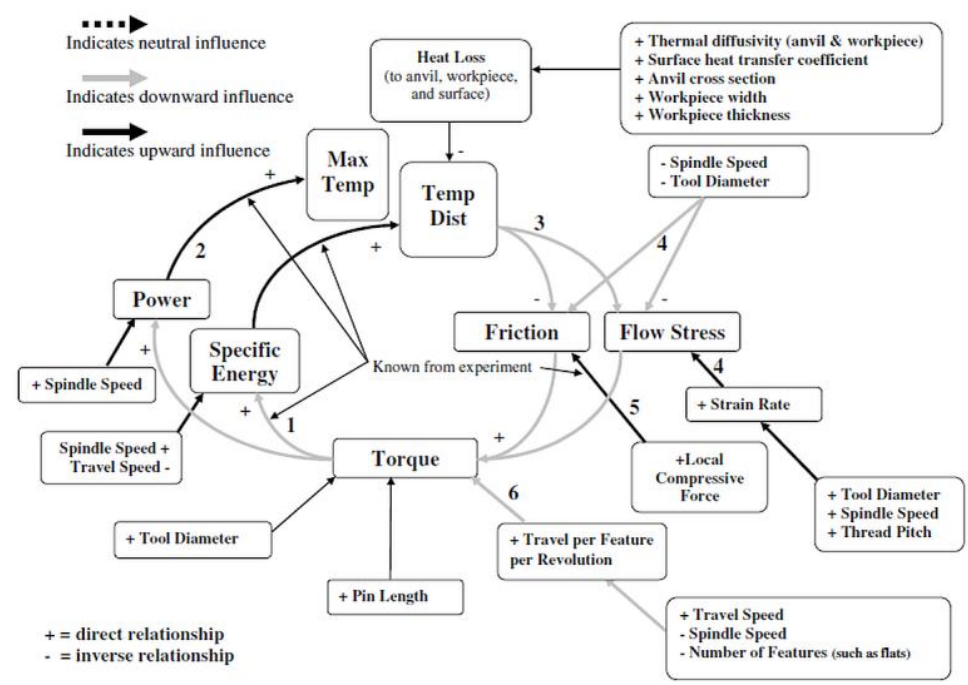

Fig. 8: Influence between variables for increasing spindle speed (Colligan, 2008). 


\section{II.3. Mechanical aspects}

During FSW, the tool / material interaction generates stresses on all the axes of the welding machine. The load intensity is considerable and therefore requires the welder to use a rather robust machine. These efforts are also related to the geometry of the tool and to the parameters (velocities) optimally chosen by operator. Vilaça et al. (2007) measured the force and the torque applied by the tool device to the welded part and the associated temperature field in order to develop calibrations in the analytical iSTIR code aiming to establish the thermal efficiency and weld quality for a giving set of FSW process parameters. Su et al. (2013) proposed an indirect methodology to estimate simultaneously in a single experiment the axial force, the traverse force and the torque of the tool. This approach is based on the measurement of the electrical signal of the motors to detect and estimate forces endured by the tool in real time. This indirect approach is economical comparing to the use of load cell and has been applied on AA2024-T4 aluminium welding by Su et al. Results show that the tool torque decrease when increasing tool rotation while the increase in traverse force is linked to both welding speed and tool rotation speed. More recently, Ullegaddi et al. (2017) have also investigated the effect of shoulder geometry and surface forces on the welding of AA6082-T6 aluminium alloys through a parametric study. They conclude that concave shoulder with tapered pin provide better results despite requiring more force.

The knowledge of the forces (forces, torques, powers) endured on the different axes of the facility is required to implicitly quantify the deformation energies and to calibrate the welding models. This measure is therefore needed to optimize the FSW process. The welding force (normal force at the plane of the sheet) recorded during the process, for example, would allow to deduce the shear at the shoulder / material interface for mechanical models based on the Coulomb friction law. This example is to be considered cautiously since the effect of the pin / material interaction on the welding force is not negligible, and especially since the material is often drained vertically in the case of threaded tools. During the FSW process, the recorded forces are unsteady and partially explained by the feature of the process which is carried out in three phases (diving, welding and withdrawal). Schmidt and Hattel (2005) have investigated the load evolution during welding on a AA2024-T3 aluminium grade. Plunge period, dwell period and weld period are identified separately. Simultaneously, a thermomechanical three-dimensional model is established in order to define process parameters leading to sticking condition at the interface between probe and matrix required for the success of the joining process according to authors. Yang et al. (2008) have also developed an equipment to follow plunge vertical force in FSW and its evolution during the whole process. This approach was efficient to detect gaps between sheets in real time through a monitoring algorithm by following the sudden change in load force applied to the tool. This device enables to develop an intelligent control as a non-destructive technique to prevent welding defect as demonstrated during welding of AA2024 aluminium sheets.

During the diving phase, the rotating tool plunges into the still relatively cold material leading to a vertical extrusion of this latter around the pin. The welding force increases drastically 
until the shoulder / material contact where it reaches a peak, while the torque increases sharply. Zimmer et al. (2010) detailed precisely the various steps corresponding to the plunging stage and associated effects on load and torque endured by the tool. High temperatures, stresses development and plastic deformation are induced at this stage. Once the contact is made, the penetration movement of the tool is stopped. Zimmer et al. (2010) recommend fixing a holding time to heat the material to a maximum temperature until it reaches a viscoplastic state thanks to the interaction between the shoulder and the material. Indeed the single plunging state does not provide the heating required inside material to promote stirring. This stage corresponds to a relaxation of the efforts and a material softening allowing the beginning of the welding phase. During this latter, two controls are conceivable. In the first one, the vertical position of the tool is kept constant which is referred as a 'control in displacement'. In the second case, the welding force is kept constant which is referred to as 'control in force'. Nevertheless a steady state is rapidly achieved in both cases. This steady regime can be observed at the microstructure level along the weld joint or also on the temperatures and load recorded. Kumar et al. (2008) discussed the choice of the welding force and the tool penetration during steady regime showing its direct influence on the weld quality. During the retraction phase, the tool / material contact is suppressed leading to disappearance of the welding force.

\section{II.4. Physical phenomena}

\section{II.4.a. Microstructures}

Microstructural evolution in FSW process is highly dependent from temperature and strain evolution endured by welded materials. Indeed, heat source induced by stirring processes and plastic deformation leads to temperature changes in nugget and neighbouring domains. High heating and cooling rate are currently observed on thermocouples measurements. Fig. 9 illustrates the temperature profiles recorded at different locations perpendicular to the weld axis on AA2024 sheet in T351 state. T351 state corresponds to solution heat-treatment at $\sim 495{ }^{\circ} \mathrm{C}$, before relieve of stress through controlled stretching. A naturally age hardened at room temperature is then developed during several days.

(a)

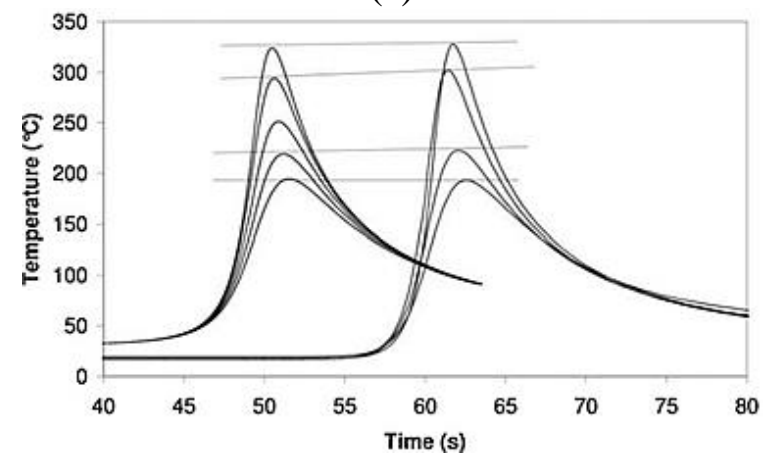

(b)

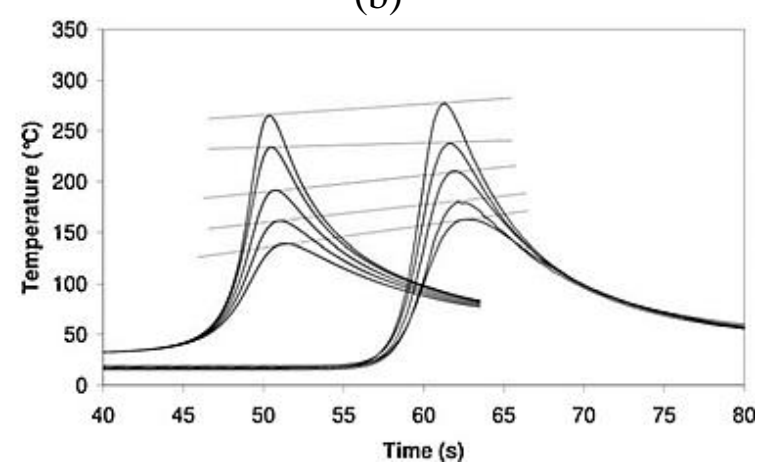


Fig. 9: AA2024 sheet, rolled down to $3.2 \mathrm{~mm}$ and welded in the T351 state. Comparison between computed (left curves) and measured (right curves) temperature cycles at the thermocouple locations (400 rpm, $400 \mathrm{~mm} \cdot \mathrm{min}^{-1}$ ) on (a) advancing and (b) retreating sides. Curves are time shifted in order to distinguish the set of results (Jacquin, 2011).

Mahoney et al. (1998) were among the first to observe and detail microstructure evolution in FSW. They describe the features in the various welded domains also considering fractures observed in tensile test. A reduction in fine hardening precipitates in the weld nugget is observed decreasing the mechanical properties. They also mentioned that larger strengthening precipitates were observed in failure domains which have been developed at the expense of the finest precipitates. More generally, they demonstrate that evolutions in microstructures features through the welding are consequences of local temperature and deformation.

Threadgill (1997) proposes a classification of the four zones of an FSW weld, each representing a type of microstructure defined by a specific heat and / or mechanical treatment. Gallais et al. (2008) show that several domains can be distinguished at the macroscopic scale when FSW has been applied on metallic alloys as highlighted on Fig. 10: 1) A central region called the stir zone (SZ) (or welding nugget) where deformation, temperature and recrystallization are the largest. This domain corresponds to the pin positions when stirring the material. According to Reynolds et al. (2000a), the width of this nugget domain is usually reported slighter greater than the pin diameter. 2) A thermomechanically affected zone (TMAZ) where grains deform and rotate as induced by the temperature evolution and mechanical deformation due to pin rotation. No recrystallization is achieved in this domain contrarily with the SZ domain according to Simar et al. (2007). However, dos Santos et al. (2013) consider that the microstructure developed in this domain has to be considered as partly recrystallized / partly recovered. Reynolds et al. (2000a) mentioned that the occurrence of recrystallization may depend from the alloy. 3) At a larger distance, a zone only affected by thermal evolutions exists where grain shape is unchanged. This heat-affected zone (HAZ) is only affected by precipitate state change due to thermal evolution. When these temperature evolutions are reduced in the metal, the base metal (BM) is retrieved.

The precipitate evolution leads to large change in mechanical properties inside the material as shown on the hardness profiles presented in the literature. Gallais et al. (2008) investigated microstructure evolution and its consequence on mechanical properties in AA6056 alloy. Legrand (2015a) have developed similar observations however restricted to the single AA2024 alloy (Fig. 11) as material of interest. In addition to microstructure evolution directly induced by FSW process, natural ageing also occurs in the weld. This evolution is mainly visible in the SZ where hardness increase is made visible after several months as observed by Frigaard et al. (2001). In order to discuss of literature models describing and predicting precipitate evolutions in FSW, a brief presentation of these evolution is proposed. Nevertheless, the stages may change depending from aluminium alloys composition, and even if similar tendencies are observed, some cautions are required in the following explanations. 


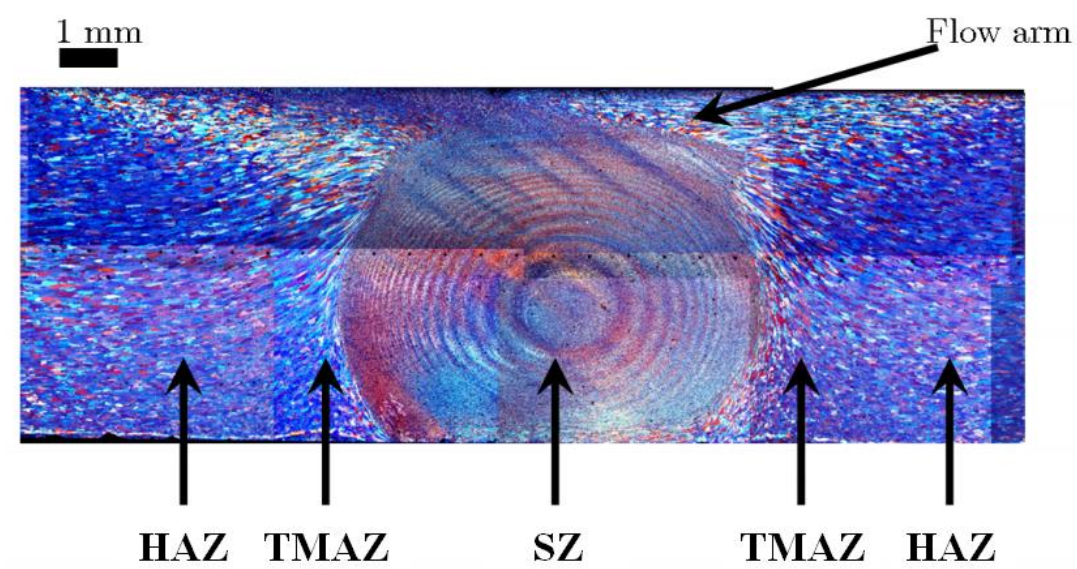

Fig. 10: Observation of FSW weld microstructure developed on AA 2024 T351 aluminium alloy (optical microscope $\backslash$ anodic oxidation) in a cross section (Genevois, 2004).

The different areas of the weld endured recrystallization or precipitation phenomena. These phenomena are closely linked to the thermo-mechanical history of the process and will have a major importance on the weld quality. Colligan and Mishra (2008) have detailed the relationships between process parameters, service life and microstructure. Optimal welding parameters have to form welds with required properties without defects as worm hole or flash. However, the process parameters (spindle speed, torque and welding speed) will significantly influence the thermal and mechanical evolution. The increase in rotational speed leads to hotter welds inducing a material softening. The flow stress will consequently be lower and friction phenomena will be exacerbated leading to an increase of the maximum temperature in the weld, also affecting the thermal cycle. The thermo-mechanical treatment provided by process itself will determine the shape and size of the affected zones considered by Threadgill (1997) where various metallurgical phenomena occur. The grain size changes depending from the strain, strain rate and temperature endured locally also considering possible dynamic recrystallization mechanisms as detailed hereafter. These evolutions have consequences on precipitation processes and grain size evolution. Both phenomena influence hardness, tensile strength, fracture toughness, fatigue properties or corrosion resistance. Consequently, Gallais et al. (2008) considered that a detailed description of microstructure evolution in FSW is required to predict such complex properties. Zhang et al. (2016) provided also a precise description of consequences induced by microstructure changes in FSW processes as reported in literature. As a consequence, the analysis of microstructure evolution is of utmost importance in order to determine end-use properties of welded parts also including effects of process parameters. The main features of the two phenomena associated to microstructure evolutions are detailed thereafter.

\section{II.4.b. Precipitation}

The SZ domain shows usually a plateau in hardness evolution. Mishra and Ma (2005) provide a detailed review description of peak temperature registered in various welding conditions and 
various aluminium grades. Maximum temperatures were usually localized in the stir zone despite the difficulties to develop relevant measurements in this domain as discussed previously. Temperature is also usually considered as quite homogeneous in this stirred domain. The maximum process temperature was reported between $400{ }^{\circ} \mathrm{C}$ and $480{ }^{\circ} \mathrm{C}$ on 7075Al-T651 aluminium alloy and restricted to around $400^{\circ} \mathrm{C}$ in AA6061 and AA6063 grades. However, more recent observations developed with thermocouples provide temperature estimation at around $450{ }^{\circ} \mathrm{C}$ in the $\mathrm{SZ}$ on this latter aluminium grade. Clear increase in peak temperature is also reported on various ranges of aluminium alloys when increasing the ratio between tool rotation and traverse speed. This large temperature increase leads to dissolution of pre-existing Guinier-Preston-Bagaryatsky (GPB) precipitates. However the large cooling rate prevents afterward the development of new precipitates. Consequently, no precipitate is observed inside the grains even using TEM facility or only with low volume fractions. The alloying elements are solutionnized in the metal. Feulvarch (2012) mentioned that a large evolution in grain size is also commonly observed as induced by recrystallization with final size of the order of $2 \mu \mathrm{m}$. A final grain size of $5 \mu \mathrm{m}$ was assumed by Kamp et al. (2007) when modelling microstructural evolutions on AA 7XXX alloy with an initial grain size of $60 \mu \mathrm{m}$. Final grain size of the order of $10 \mu \mathrm{m}$ were reported by Fratini and Buffa (2005) on AA6082 T6 aluminium grades for an initial grain size close to $50 \mu \mathrm{m}$. The end-use hardness is usually obtained in this nugget region after natural ageing. Indeed, Gallais et al. (2008) observed that the hardness level is similar to the one encountered in the base metal on AA6056 aluminium grades when natural ageing has occurred.

(a)

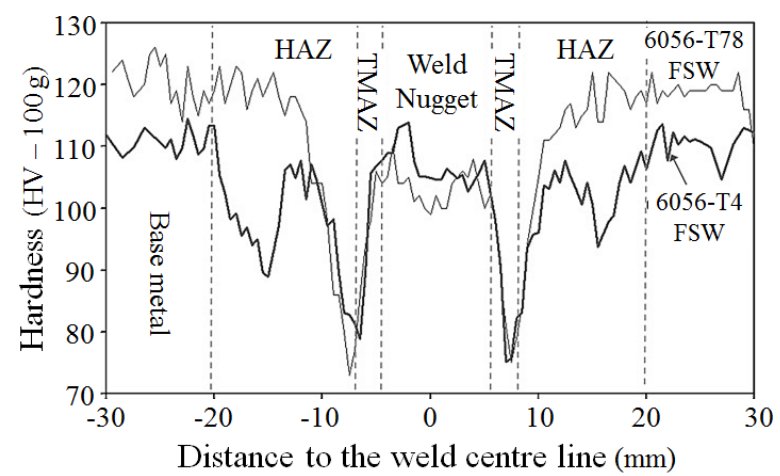

(b)

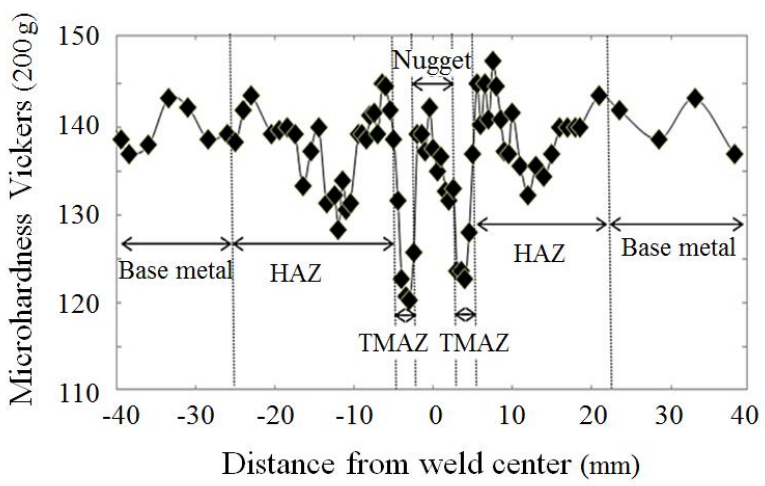

Fig. 11: Hardness profile evolution obtained after FSW process on the cross-section of (a) a AA6056 aluminium alloy in T4 and T78 state (Gallais et al., 2008) and (b) a AA2024 aluminium alloy in T3 state (Legrand et al., 2015b).

The TMAZ domain is highlighted with a clear hardness decrease. In AA6056 alloys - T4 state (solution heat treated and naturally aged), elongated Q precipitates develop on dispersoids among grains. These latter are observed enriched in Mn elements. GPB precipitates also develop during natural ageing explaining a large part of the measured hardness. In AA6056 alloys in $\mathrm{T} 78$ state (solution heat treatment at $550^{\circ} \mathrm{C}$, air quenching, tempering during 8 hours at $175^{\circ} \mathrm{C}$ and final overaging at a temperature higher than $175^{\circ} \mathrm{C}$ ) similar evolutions are 
observed. As mentioned, recrystallization does not occur as deformation is too weak in this domain. However, some precipitates dissolve. The lowest hardness is usually obtained at the boundary between the TMAZ and HAZ domain (Fig. 11) where a sharp decrease is observed. Gallais et al. (2008) considered that this drop is directly related to the heterogeneous development of coarse precipitates located both on dislocations and dispersoids. This nucleation is without substantial effect on the hardness properties explaining the low values measured in hardness profile. According to Dos Santos et al. (2018), the lack of supersaturation induces by the development of these coarse precipitates prevents any future natural ageing and nucleation of GPB phases.

The HAZ is characterized with a non-monotonous evolution in hardness. Indeed, a decrease in hardness is initially observed at large distance (Fig. 11) on various aluminium alloys. Gallais et al. (2008) reported such observation on AA6056-T4 aluminium grades and explained this evolution as induced by the dissolution of GPB precipitates in aluminium matrix. Indeed, DSC observations developed by Gallais show a clear exothermic peak associated to GPB reprecipitation when analysing samples extracted from HAZ. Legrand et al. (2015b) also reported similar observations and same conclusions on AA2024 samples. Genevois et al. (2005) considered that precipitates may coarsen or dissolve in the HAZ leading to hardness decrease regarding base metal properties. Dixit et al. (2009) have similarly developed careful DSC experiments on samples extracted from HAZ and SZ after FSW applied on an AA2024T3 sheet. Large decreases in the amount of GPB zones are observed when both peak temperature and duration of cycles increase. Interestingly, the coarsening of $S$ phase precipitate in HAZ zone is measured by TEM observations showing smaller evolution compared to the similar one reported in SZ. According to Dixit et al. (2009), the maximum temperature is in the range $250-350{ }^{\circ} \mathrm{C}$ in this specific domain. The increase observed at a smaller distance from the weld centre line is related to the development of tiny precipitates in a heterogeneous nucleation process localized on dislocations. These precipitates have some influences on the increase of hardness mainly for the largest temperatures evolution. This evolution is discussed in details by Legrand (2015a) when considering the simulation of the S-phase development induced by the high temperatures reported close to the TMAZ domain.

\section{II.4.c. Grain evolution}

The intense thermomechanical history undergone by the material during welding and more particularly in the stir zone produces a deep transformation of the microstructure as reported in experimental observations (Fig. 12). Thus, a complete recrystallization (development of refined, equi-axed and homogeneous grains) occurs in the nugget and the precipitate dissolution and coarsening take place within and around the stirred zone. Fig. 12 also demonstrates that the width of the nugget domain depends from the depth inside the material. Indeed, the width of the recrystallized domain evolves from 2 to $6 \mathrm{~mm}$ in this example (Fig. 12). This evolution of the stirred domain is probably induced by the shape of the tool and also due to the complexity of the stirring process inside material. 


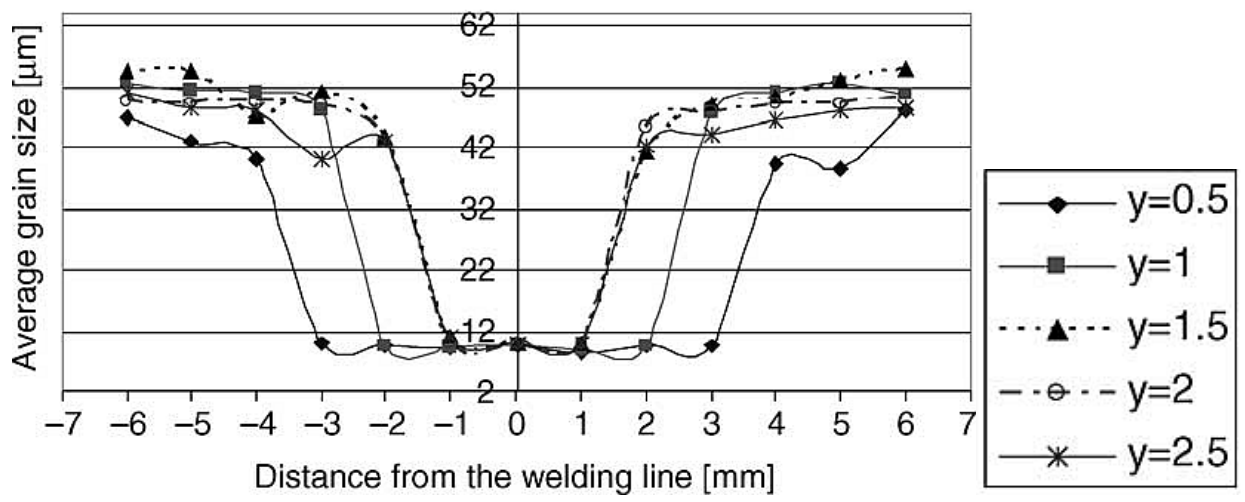

Fig. 12: Grain size evolution depending from the distance to the weld centreline. Evolutions are shown for various vertical position, $\mathrm{y}(\mathrm{mm})$, measured from the upper surface (AA6082 T6 - $715 \mathrm{rpm} / 200 \mathrm{~mm} \cdot \mathrm{min}^{-1}$ ) (Fratini and Buffa, 2005).

Many authors have observed and studied the grain refinement process in the SZ. Jata and Semiatin (2000) investigated Continuous Dynamic Recrystallization (CDRX) phenomena during friction stir welding of high strength aluminium alloys. They showed that the original grains and subgrains boundaries are replaced with fine, equiaxed recrystallized grains in the weld nugget. This proves that dynamic recrystallization by a discontinuous process is not possible. Indeed, microstructures observations carried out by Jata and Semiatin does not show the recrystallization nuclei formed and gross grain-boundary migration occurring usually during the discontinuous dynamic recrystallization (DDRX). Instead, the OIM measurements performed by Jata and Semiatin (2000) revealed that the magnitude of the misorientations increased significantly during FSW (Fig. 13) when compared with the base metal. The authors conclude that the grains observed in the nugget are consequently high-misorientation subgrains. This phenomenon is typically observed for Continuous Dynamic Recrystallization (CDRX) microstructure, similarly to microstructures leading to subgrain development during hot rolling. Gourdet and Montheillet (2003) have presented a careful description of this phenomenon.

(a)

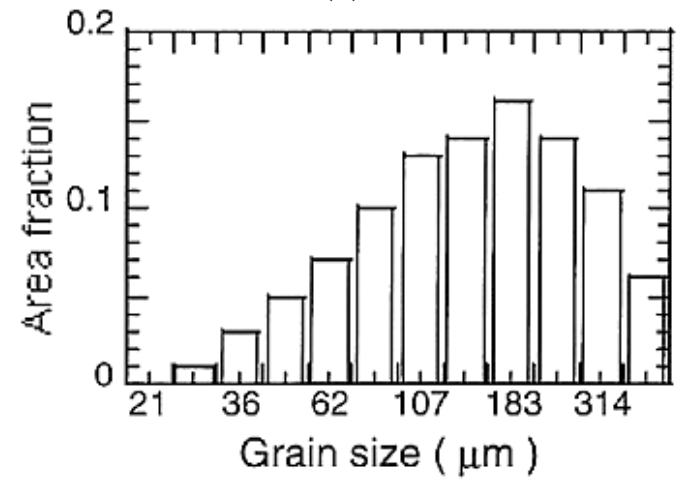

(b)

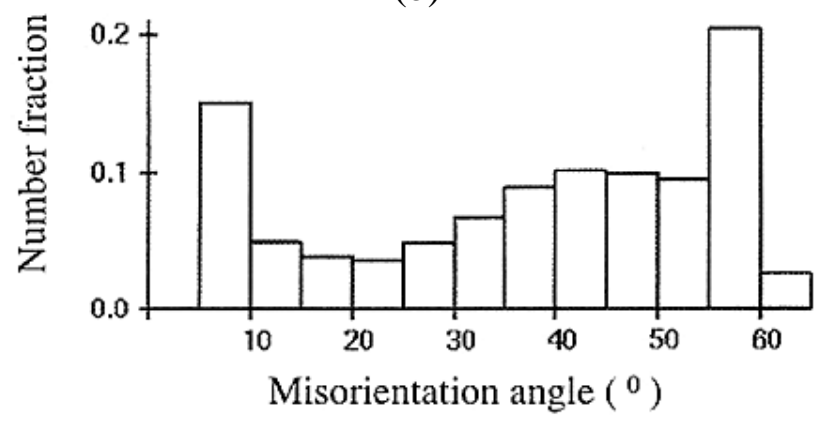

Fig. 13: Al-Li microstructures: OIM results (a) grain size distribution and (b) number fraction versus misorientation angle between grains (Jata and Semiatin, 2000). 
Other authors also assume that CDRX occurs during FSW/FSP. Mishra and Ma (2005) propose a summary of the grain size evolutions in the nugget zone of FSW/FSP aluminium alloys (Table 3). Consequently, the size of recrystallized grains in the FSW/FSP aluminium alloys usually increases when increasing the tool rotation or the ratio between rotation rate and traverse speed. It has to be noticed that the postdynamic thermal cycle can have a significant effect on the final recrystallized grains size considering that a slow cooling may produce a remarkable grain growth after stirring.

\section{Modelling and simulation}

Several models have been proposed in the past years to simulate microstructure evolutions in metallic alloys during FSW process and the associated hardness profile. These models aim at determining the final properties of joined materials and the beneficial/detrimental effects of FSW process on these latter. Approaches are generally developed at specific scale depending from the objectives and ideas of authors behind the model. In addition, the approaches mainly reported in the literature can be classified in two main classes as summarized in Table 2-3. The first approaches are dedicated to precipitation modelling. They generally aim to follow evolution of precipitates dispersed in aluminium matrix at micro-scale. In such approaches, the evolution of the precipitate size distribution (PSD) induced by temperature and heat evolutions is followed during FSW process. These models generally derivate from the one originally proposed by Wagner and Kampmann (1991) and its implementation by Myhr and Grong (2000). However, some rough analytical expressions have been proposed by some authors to estimate precipitate distribution evolution using Time Temperature Transformation (TTT) diagrams. At a meso-scale, research activities also focus on the grain structure evolution induced by recrystallization mechanisms. Even if only the SZ endures recrystallization mechanism as mentioned previously, it appears that authors have also frequently extended their approach to model grain structure evolution in TMAZ and HAZ where no recrystallization mechanism occurs. These various approaches will be detailed hereafter with their associated results.

The determination of the final hardness profile is not the sole objective of microstructural modelling approaches reported in literature. As pointed out by Kamp et al. (2006), the determination of the grain structure and/or precipitate distribution after welding is also of clear interest. As an example, the nature of final precipitates and their associated distributions are of importance to determine other properties associated to the welded domain and linked to the in-service strength of pieces. More complex properties such as corrosion resistance, fracture toughness, fatigue life or resilience can be mentioned as discussed by Gallais et al. (2008). Nevertheless, all models have to couple heat resolution and precipitation models. The first ones provide the temperature evolution through the HAZ, TMAZ and SZ and second ones its effect on precipitates features. In addition, the final thermomechanical properties (strength and hardness) are usually provided by a semi-empirical model based on precipitate fractions or precipitate size distribution and fitted on experimental comparisons. 
This approach was proposed and applied by Genevois et al. (2005). Legrand et al. (2015b) also developed similar methodologies on AA2024 aluminium grades. Models dedicated to simulation of microstructure evolution in FSW have been provided on a large range of aluminium alloys as partially detailed by Kamp et al. (2007).

\section{III.1. Molecular dynamics}

As a preliminary discussion on microstructure evolution at micro-scale, researches based on estimation of atoms displacement in FSW processes have to be highlighted. Indeed, some rare studies report the possibility to develop defect analyses based on molecular dynamics simulation. Through an atomic scale modelling, Dmitriev et al. (2014) investigated the basic mechanisms at the onset of structural inhomogeneity in FSW when large plastic deformation occurs. Motion of atoms induced by tool movement is simulated leading to nonequilibrium states in the crystal lattice which are analysed and discussed. Similarly, Nikonov et al. (2015) carried out also some investigations using similar numerical approaches, in loading conditions closed to the ones encountered in FSW processes. Intermixing of dissimilar atoms is observed and measured through the welded domain. Nevertheless, in such applications, the nature and properties of materials have not been deeply analysed as well as their influence on process evolution. However effects of similar $(\mathrm{Cu} / \mathrm{Cu})$ and dissimilar $(\mathrm{Cu} / \alpha-\mathrm{Fe})$ welding have been investigated by Nikonov et al. (2015) considering pairing of two crystallites. In addition, some developments have been done on two crystallites of aluminium 2024 as aluminium alloys of industrial interest.

\section{a. Presentation}

The general aim of the authors is to investigate basic mechanisms at the onset of the structural state generation in materials subjected to large and severe plastic deformation as the one observed in FSW processes. Modelling of atoms movement are proposed in order to describe, analyse and report atoms evolutions at narrow scale during stirring processes and mixing of materials. All simulations were conducted in the framework of molecular dynamics methods with the use of the commercial software package LAMMPS (LAMMPS, 2019). The modelling of atom interactions is described using the usual formalism. However, the domains of interest (i.e. crystallites) are at the nanometre scale and the linear and rotational velocities of the tool are adapted to such scale. Considering these hypotheses, the investigated 3D domains in first simulations are of $10.8 \times 21.7 \times 3.6 \mathrm{~nm}^{3}$, and the cylindrical or conical tool diameter is of $3.6 \mathrm{~nm}$ with a constant rotational velocity of $\omega=0.1 \mathrm{ps}^{-1}$. The feeding rate is consequently also larger and equal to $v=50 \mathrm{~m} \cdot \mathrm{s}^{-1}$. Such molecular dynamics approach requires removing the heat introduced into materials by the rotating tool to prevent artificial increase in total energy. Consequently, an artificial viscosity of atoms belonging to two buffer layers on the edges of both domains is introduced. In addition, some periodic boundary 
conditions were also set in all directions to account for extended sizes of the samples. As mentioned previously, similar and dissimilar metallic solutions were both investigated.

\section{b. Results}

A first simulation was proposed by Nikonov et al. (2015) in order to reproduce atoms movement induced by mixing when cylindrical tool pass between two inter-crystallite boundaries of similar grain orientation. Copper material has been chosen in this first case and new positions of atoms are illustrated on Fig. (14 a) with various shades of grey showing movements of material. The thickness of area impacted by FSW is of the order of the tool size $(\sim 3.6 \mathrm{~nm})$. At a larger distance, atoms still occupy their original position. The spatial distribution of atoms in the two metals and structural defects is provided on Fig. (14 b) in the $\mathrm{X}$ direction. Material is moved toward the right hand side part as a combination of rotational and feeding rate direction. Analysis by using the common neighbour method has been developed however revealing a low amount of structural defects in pieces. Indeed, original local topology is well preserved as expected in the neighbourhood of original boundaries.

Another simulation is proposed by Nikonov et al. (2015) dedicated to aluminium alloys as an extension of the previous simulation showing the ability of molecular dynamics methods to model structural changes. This case is dedicated to a 2024 industrial aluminium grade with change in tool geometry. Indeed, a conical tool is used and similar rotational velocity and feeding rate are applied compared to first simulation. Both crystals have a dimension of $12.2 \times 24.4 \times 2.03 \mathrm{~nm}^{3}$ with same crystallographic orientation.

(a)

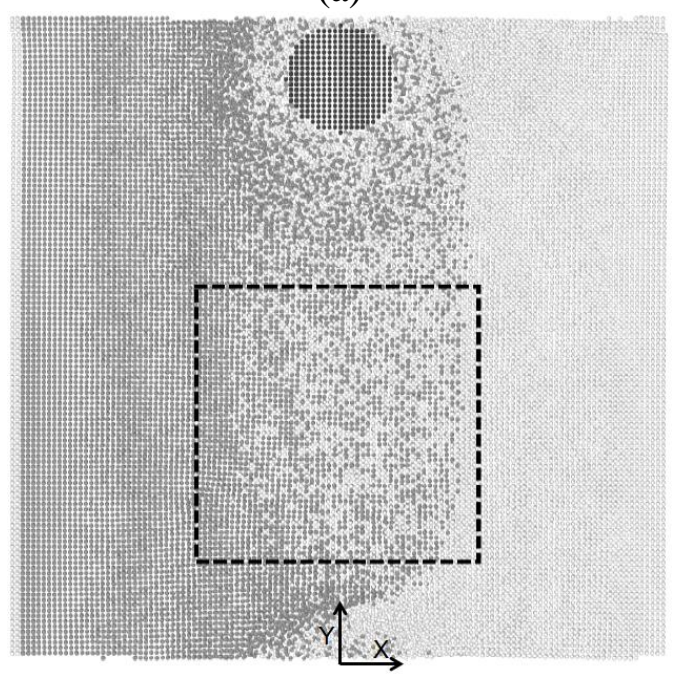

(b)

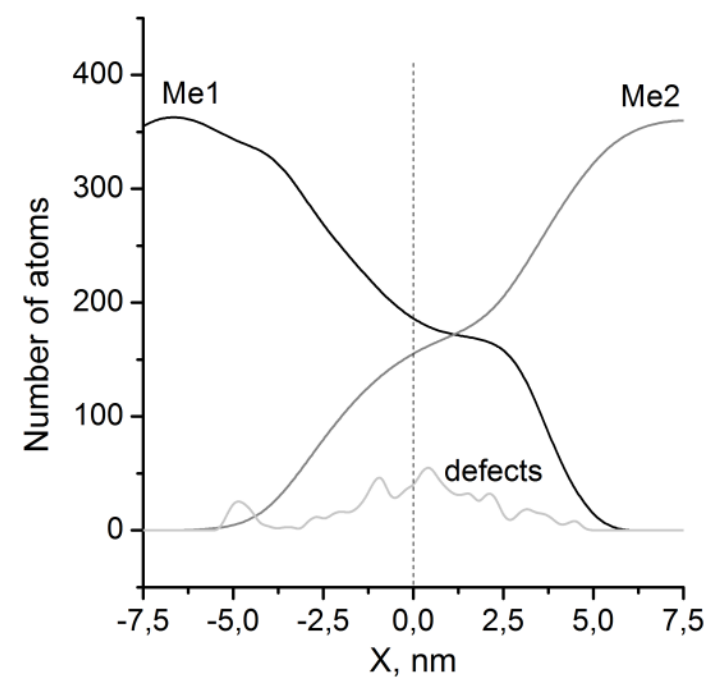

Fig 14: (a) Projection of atoms in horizontal plane after welding on conjugate $\mathrm{Cu}-\mathrm{Cu}$ crystal. (b) Spatial distribution of atoms in the square domain (dotted line in a) showing the defects in microstructure (Nikonov et al., 2015). 
The movement of atoms is well reproduced (Fig.15 a) showing the nugget domain with the mixing of two AA2024 materials. The progressive decrease of atoms fraction of one crystallite to another with the distance to the weld centre line is highlighted. No clear mixing is observed at a distance larger than the rotating tool. The influence of vibration impact in the vertical direction is similarly investigated showing some effects on the introduction of chemical element. As a secondary step, Nikonov investigates the influence of additional vibration impact applied to the conical indenter with an amplitude of $0.15 \mathrm{~nm}$ and a frequency of $0.1 \mathrm{ps}^{-1}$. This oscillating impact aims at investigating the implementation of atomic mechanism under loading conditions identical to FSW process. Fig. 15 b) shows the distribution of atoms in the two crystals on AA2024 aluminium alloy after FSW process in both cases. Considering vibration impact, the penetration of atoms of one crystallite to another is increased by about $20 \%$ compared with simulation developed without vibration impact. This effect seems mainly visible in the retreating side (left hand side on Fig. 15 b). The use of vibration leads to a more uniform introduction of the elements of the opposite plate in the weld zone considering a vertical cross section. According to authors, this use should increase the bond strength along weld line. Nikonov et al. (2016) have proposed more recently some extensions of this simulation in order to investigate influence of increased vibration frequency and amplitude also considering two crystallites of same compositions. These calculations demonstrate that increasing the vibration frequency to $1.0 \mathrm{ps}^{-1}$ improves the penetration depth of atoms from one crystallite to another in the interfacial region. The increase of vibrations amplitude by a factor 5 similarly increases the penetration of atoms with same amount. According to the authors, ultrasonic vibrations applied to a FSW tool in direction parallel to its rotation axis leads to a uniform penetration of elements from one material to the other enhancing the bond strength of the joined domain.

(a)

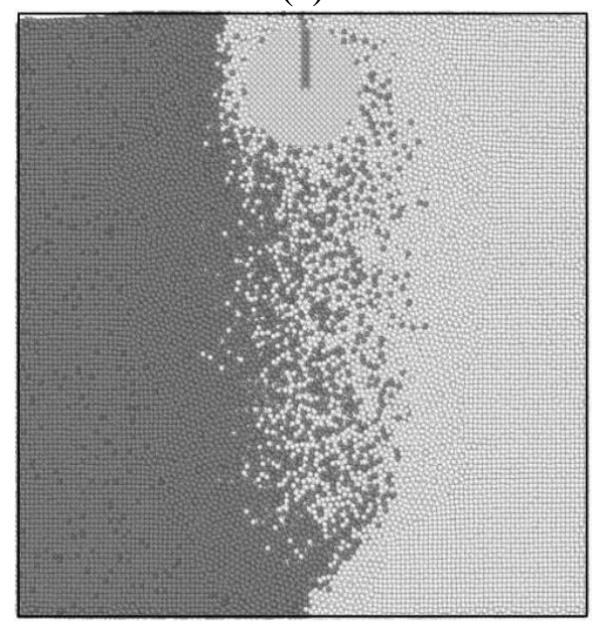

(b)

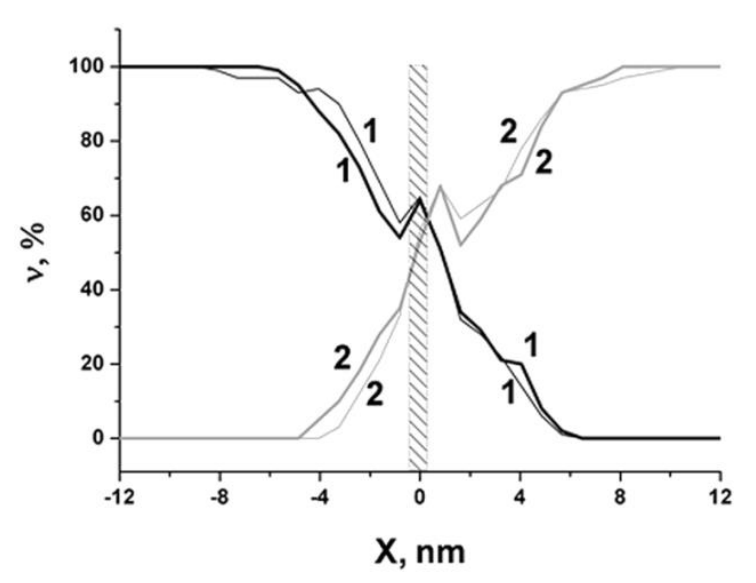

Fig. 15: (a) Atoms projection on the horizontal plane at the end of FSW process applied on AA2024 aluminium alloy $\left(v=50 \mathrm{~m} \cdot \mathrm{s}^{-1}\right.$ and $\left.\omega=0.1 \mathrm{ps}^{-1}\right)$. Copper atoms are shown with darker marks. (b) Distribution of atoms along the $X$ (horizontal) direction. Curves correspond to atoms of left (1) and right (2) hand-side crystallites. The thickness of curves corresponds to welding processes without (thin) and with (fat) vibration impact. (Nikonov et al., 2015) 
According to Dmitriev et al. (2014) and Nikonov et al. (2015, 2016), the present computer model should provide better understanding of the basic laws on the origin of structural inhomogeneity in FSW processes. As a conclusion, it cannot be deny that some difficulties may arise to integrate results of molecular dynamics simulations at macro-scale. This point is a discussion topic also in literature. Indeed it may be difficult to link process parameters used in molecular dynamics simulations to realistic process values. Consequently the investigation based on such approach on the effect of FSW process conditions on weld quality and defect occurrence is complex as well as the optimisation of welding parameters. Despite this limitation, even if few studies based on atomistic modelling are dedicated to the simulation of microstructure evolution in FSW, these approaches should not be disregarded. Indeed they gave the opportunity to follow the early stages of precipitate development such as the cluster and GPB zones nucleation. In addition, it should be emphasized that these methods can also consider the elastic deformation effect onto the matrix and precipitate and their effect on precipitation process. We may have also to consider that future development will be induced by the current progress in computer science and increase of computational capacity.

\section{III.2. Precipitation modelling}

The models reported in the literature to follow microstructure evolution in FSW processes are of various types, depending from the scale of analyses and expected prediction. We may have to distinguish these latter in various types considering the chosen numerical approaches. The table 2 summarizes these approaches with associated assumptions and remarks for various models recently reported. 
Table 2: Models dedicated to the simulation of precipitate evolutions in FSW processes as applied on aluminium alloys:

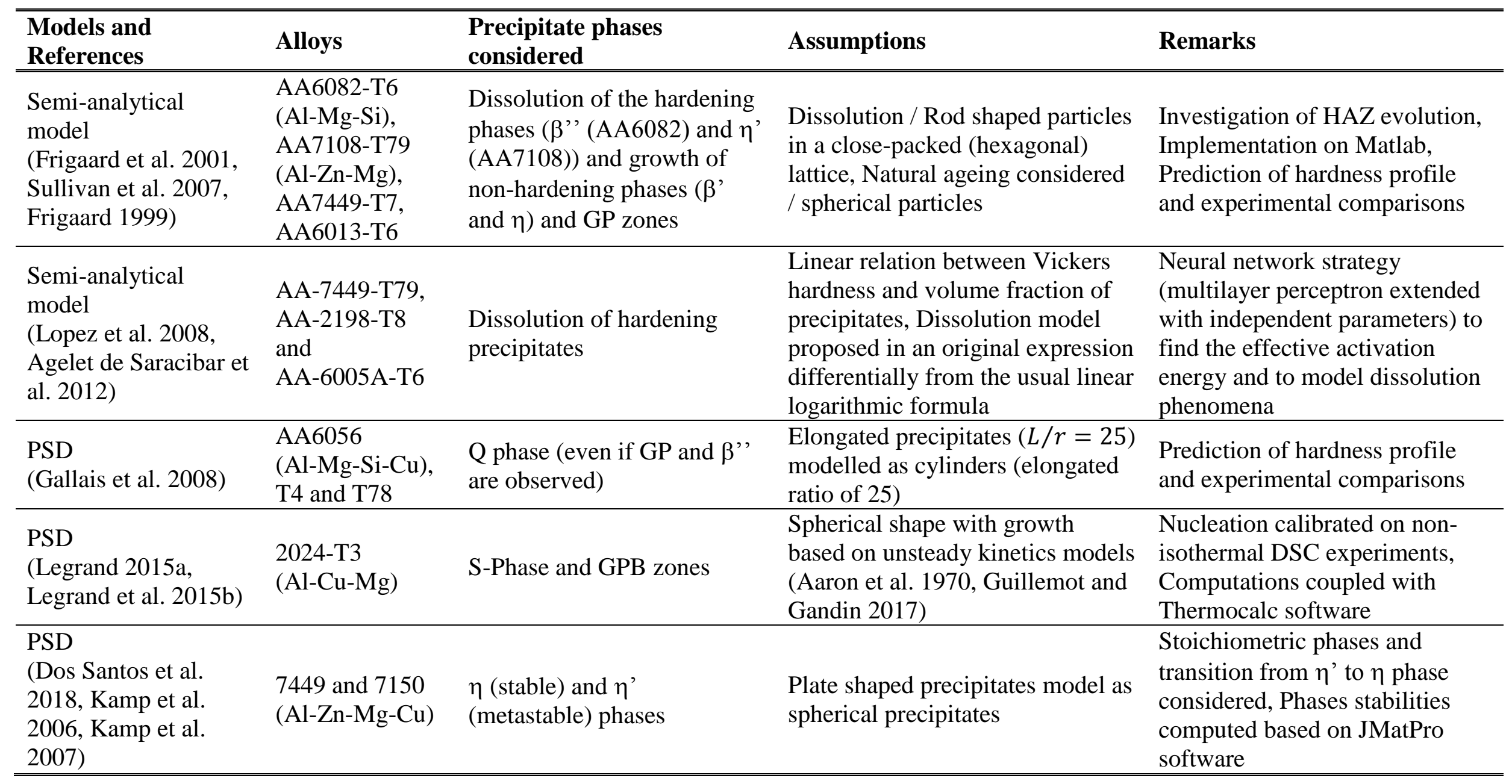




\section{III.2.a. Semi-analytical model}

Several models are reported in literature enabling to follow phase fractions evolution during FSW process based on additive approaches which are assumed as semi-analytical. Simar et al. (2012) named these latter as 'Internal variable models' as based only on the numerical integration of the thermal cycles endured by the material. In such model, the temperature evolution is consequently preliminary estimated at macro-scale using thermo-mechanical simulations of FSW process or even analytical estimation. Precipitate evolutions are then computed step by step and based on an integrative approach. The temperature evolution is used as an input in order to define increase or decrease of precipitate fractions during infinitesimal time step. Following the idea proposed by Myhr and Grong (Myhr et al., 1997), the dissolution model is kept as simple as possible in order to couple precipitates evolution to FE simulation.

\section{a. Presentation}

The models reported in the literature and based on integrative approaches usually rely firstly on the estimation of an equivalent time, $t_{r}$, corresponding to complete dissolution of precipitates during welding. This time is reported on master curves providing the decrease in hardening precipitates and associate mechanical properties for a given time. Natural ageing is then considered as a second step and leads to the possibility to develop other hardening precipitates. Such approaches have been extensively detailed by Myhr and Grong and their co-authors to provide experimental and numerical strategies for the estimation and exploitation of master curves. Myhr et al. (1997) investigated the thermal stability of hardening precipitates and effect of initial precipitates sizes and composition to define optimized welding conditions for Al-Mg-Si alloys. Myhr et al. (1998) similarly applied this model to describe the thermo-mechanical evolution of welded material also considering microstructural evolution. Applications of both activities were focused on AA6082 aluminium grades. Finally, Bjørneklett et al. (1999) investigated dissolution and aging kinetics in the heat affected zone during welding processes in Al-Zn-Mg aluminium alloys with the objective to propose constitutive equation of phase fraction evolution in a differential formulation. These activities all rely on the definition, computation and use of master curves based on experimental observations when applying isothermal heat treatment on metallic samples. Hardness measurements provide consequently access to the rate of precipitate dissolution and associated time. Frigaard et al. (1999) have applied this methodology in the modelling of precipitate evolution in FSW processes. This latter has provided a detailed and precise description of this methodology in his Ph.D. Thesis (Frigaard, 1999). The aim of Frigaard was to provide thermal field evolution and final hardness profile for a large set of process conditions applied on Al-Mg-Si and Al-Zn-Mg aluminium alloys. Sullivan et al. (2006) has afterwards also developed similar methodology. Interesting results have been obtained in the 
prediction of hardness evolution and strength loss in a profile of welded joint also compared with experimental measurements.

\section{b. Methodology}

The methodology has been firstly developed in order to model precipitate dissolution of hardenable precipitates. Myhr et al. (1997) consider the dissolution kinetics of platelike precipitates expressed with the following formula:

$\frac{\mathrm{d} f}{\mathrm{~d} t}=-\frac{f_{0}}{B_{0} \sqrt{\pi}}\left(\frac{C_{i}-C_{m}^{0}}{C_{p}-C_{m}^{0}}\right)\left(\frac{D}{t}\right)^{1 / 2}$

where $f$ and $f_{0}$ are the current and initial particle volume fraction, $B_{0}$ is the half thickness of the plate, $D$ is the diffusion coefficient. $C_{i}$ and $C_{p}$ are respectively the matrix and precipitate composition at the interface. $C_{m}^{0}$ is the composition at large distance from the interface. The solute profile is schematized on Fig. 16 a). Similar methodology is also provided by the authors for spherical precipitate of radius $r$ with the following expression:

$\frac{\mathrm{d} r}{\mathrm{~d} t}=-\left(\frac{C_{i}-C_{m}^{0}}{C_{p}-C_{m}^{0}}\right)\left[\frac{D}{r}+\left(\frac{D}{\pi t}\right)^{1 / 2}\right]$

The equation (1) can consequently be developed after simplification in order to provide time required for complete dissolution of plate and spherical precipitates respectively expressed as:

$t_{1}^{*}=t_{r 1}\left(\frac{C_{i}^{r}}{C_{i}}\right)^{2}\left(\frac{B_{0}}{B_{0}^{r}}\right)^{2} \frac{D_{r}}{D}$

$t_{2}^{*}=t_{r 2} \frac{C_{i}^{r}}{C_{i}}\left(\frac{r_{0}}{r_{0}^{r}}\right)^{2} \frac{D_{r}}{D}$

where $t_{r 1}$ is the reference time required for complete dissolution of platelike precipitates with interfacial matrix composition $C_{i}^{r}$, size $B_{0}^{r}$ and diffusion coefficient $D_{r}$. Similar expression (Eq. 4) is provided for spherical precipitates with initial radius $r_{0}$ when considering a reference time required for complete dissolution equal to $t_{r 2}$. Some other direct expressions are also provided by Myhr et al. (1997) for estimating dissolution time at any given temperature $T$ also considering evolution of dissolution coefficient. From the previous relations, direct expressions of precipitate fraction, $f$, can be deduced considering initial fraction $f_{0}$ and current time $t$ for respectively plate and spherical precipitates:

$\frac{f}{f_{0}}=1-\left(\frac{t}{t_{1}^{*}}\right)^{1 / 2}$
$\frac{f}{f_{0}}=\left(1-\frac{t}{t_{2}^{*}}\right)^{3 / 2}$

Myrh et al. (1997) proposed to use dimensionless times (Eq. 5-6) as well-suited variable to eliminate unknown kinetic constants. By raising the dimensionless time to a specific power 
$(1 / 2$ or $3 / 2)$, the premultiplying constant is also independent of the exponent value and is itself dimensionless. Fig $16 \mathrm{~b}$ ) shows the variation in logarithmic axes of the ratio fraction for plate precipitates in AA2024-T6, AA6013-T6 and AA7449-T6 aluminium alloys measured after isothermal treatments. These curves are similar to the ones provided by Myhr et al. (1991) using hardness measurements to evaluate the ratio of residual fraction of precipitates. The exponent is seen to fall to lower values compared to expected slope for large fraction of dissolved precipitate. This evolution is explained by interactions between neighbouring particles and impingement of diffusion fields. The approach of Myhr et al. (1997) is then based on the demonstration that precipitate dissolution is an isokinetic transformation considering an intermediate value, named $I$. As an example, we obtain for plate precipitates:

$\frac{d f}{d t}=\left(\frac{d f}{d I}\right)\left(\frac{d I}{d t}\right)=\left(\frac{d f}{d I}\right) \frac{1}{t_{1}^{*}}$

Variable $I$ is expressed as the time-integration of inverse of the current dissolution time, $t_{1}^{*}$ :

$I=\int_{0}^{t_{1}} \frac{d t}{t_{1}^{*}}$

(a)

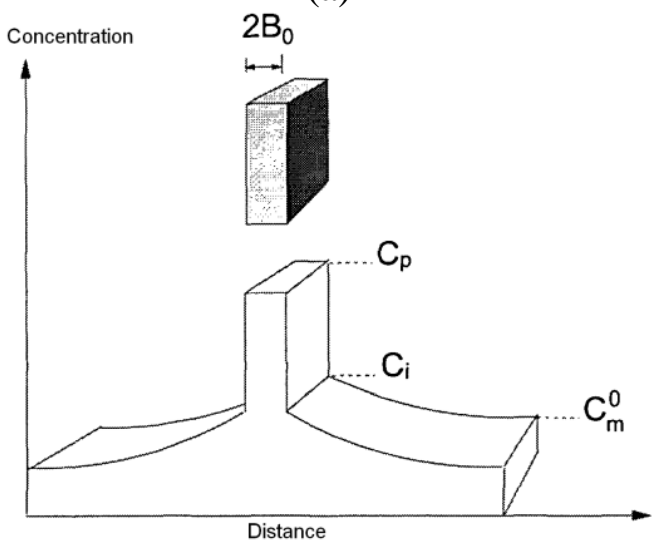

(b)

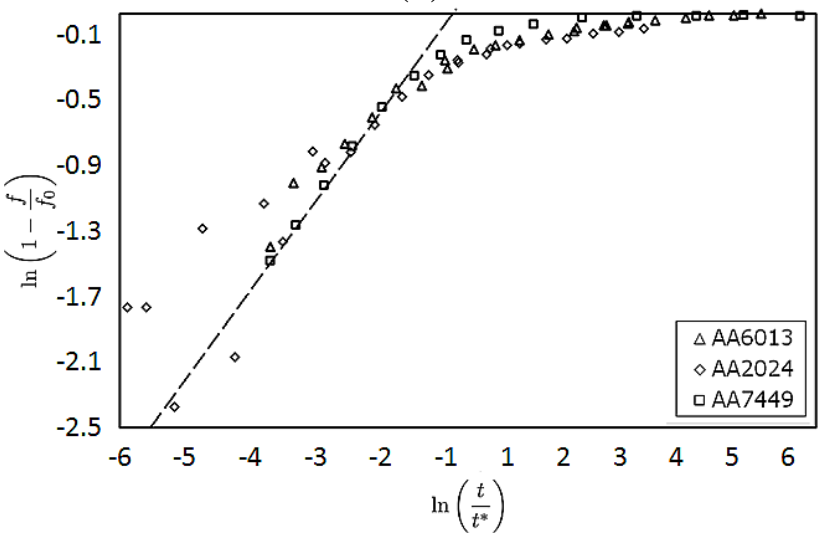

Fig 16: (a) Schematic representation of concentration profile around platelike (i.e. 1D) precipitate with half-thickness $B_{0}$. Concentration in precipitate and matrix at the growing interface are respectively equal to $C_{p}$ and $C_{i}$. Concentration at large distance remains equal to $C_{m}^{0}$ (Myhr et al. 1997). (b) Master curves for AA2024-T6, AA-6013-T6 and AA7449T7 aluminium alloys with analytical evolution (dashed line) (Sullivan et al. 2006).

This integral (kinetic strength (Myhr et al., 1997)) is then estimated during the investigated thermal cycle in order to provide numerical estimation of the $I$ variable. This value then replaces the time ratio shown in Eq. 5 and provides direct access to residual fractions of precipitate also considering master curves presented in Fig 16 b). This latter figure shows the calibrated master curve gathering the three aluminium grades investigated by Sullivan et al. (2006), each alloy having its own individual activation energy and temperature for complete dissolution, $t_{1}^{*}$. Consequently, for any temperature evolution, this methodology gives access to the precipitate fraction during dissolution stage as long as local expression of dissolution time, $t_{1}^{*}$, is accessible or estimated. In addition, a natural ageing component is added by Myhr 
et al. (1997) to predict final hardness at room temperature. Sullivan et al. (2006) developed a slightly different approach. The first authors consider a simple reversion of the dissolution model and apply it to growth stage. From Eq. (1), they may deduce a similar expression compared to the ones produces for dissolution:

$\frac{B_{0}}{B_{0}^{r}}=\frac{C_{m}^{0}-C_{i}}{C_{m}^{0, r}-C_{i}^{r}}\left(\frac{D_{a} t_{a}}{D_{a, r} t_{a, r}}\right)^{1 / 2}$

where index $r$ refers to the reference alloy. The ratio of precipitate size is consequently a function of the aging temperature $T_{a}$, in the expression of the diffusion coefficient $D_{a}$, and of the initial matrix composition, $C_{m}^{0}$, at the beginning of the natural ageing process which is still to be estimated. In a quite different approach, Sullivan et al. (2006) assume that the peak temperature reach during welding dominates the final natural ageing response. Isothermal heat treatments are used in order to calibrate the model.

\section{c. Results}

This latter model has been applied by Frigaard et al. (2001) to predict microstructure evolutions in HAZ and to propose comparisons with experimental observations. These comparisons were also dedicated to the validation of the heat flow model introduced to predict temperature evolution during welding. Microstructure evolutions are mainly based on Vickers hardness measurement. Two aluminium grades, AA6082-T6 and AA7108-T6 were firstly investigated by authors. Comparison between measurements and simulations are reported in Fig. 17 for the two aluminium grades and for two linear velocities. The process model predicts accurately the response of the base material as shown in comparisons. Similar hardnesses are predicted with the expected profile. A clear decrease in hardness is observed in the HAZ due to the partial dissolution of hardening phase ( $\beta^{\prime}$ ' and $\eta^{\prime}$ respectively) in the two grades with growth of non-hardening phase ( $\beta^{\prime}$ and $\eta$ respectively) during the cooling leading to solute depletion in aluminium matrix.

Some discrepancies are also observed in Fig. 17 b) (AA6082-T6) for the largest velocity due to an underestimation of solute diffusion phenomenon. Indeed, according to Frigaard et al. (2001) a short-circuit mechanism is certainly involved for this large velocity $\left(12 \mathrm{~mm} . \mathrm{s}^{-1}\right)$ when a part of the HAZ falls within the plastically deformed region beneath the tool shoulder. Dislocation will act as diffusion path (short-circuit mechanism) for atoms. A reduction of the time constant, $t_{r 1}$, is proposed by Frigaard to mimic the enhancement of dissolution process leading to better comparison in HAZ hardness (dashed line in Fig. 17 b). Good comparisons are also observed for AA7108-T6 alloy (Fig. 17 c-d). The strength recovery after welding is adequately captured after simulation. Natural ageing $(270 \mathrm{~h}$ for AA6082 and $160 \mathrm{~h} / 2600 \mathrm{~h}$ for AA7108) phenomenon is also well reproduced at ambient temperature with the associated increase in hardness in the central part of the well. The 
hardness decrease is controlled in the welded domains compared with BM after 100 days of ageing showing low evolutions of mechanical properties in hardness.

(a)

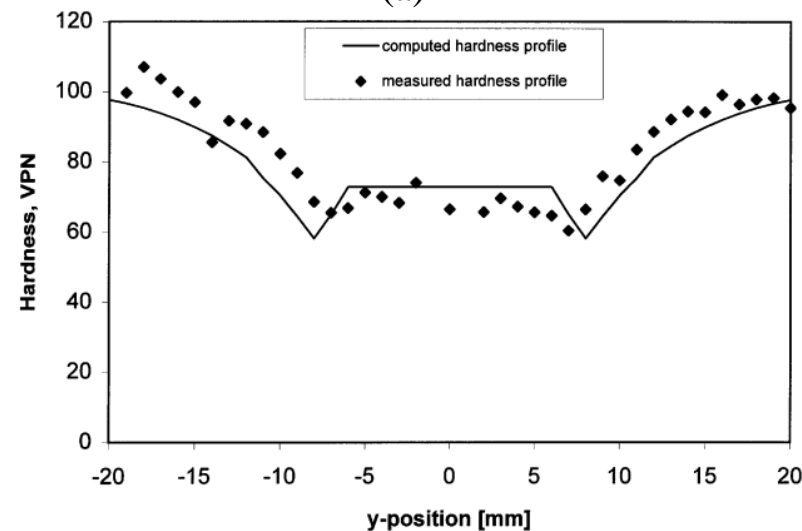

(c)

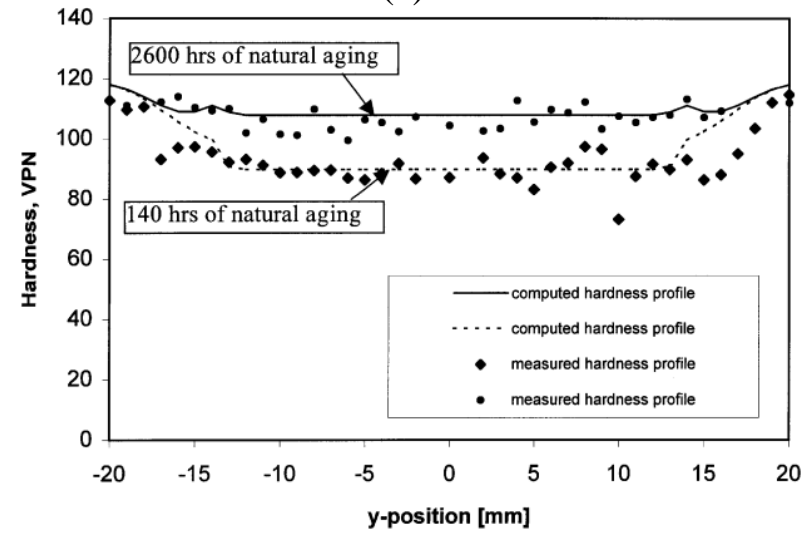

(b)

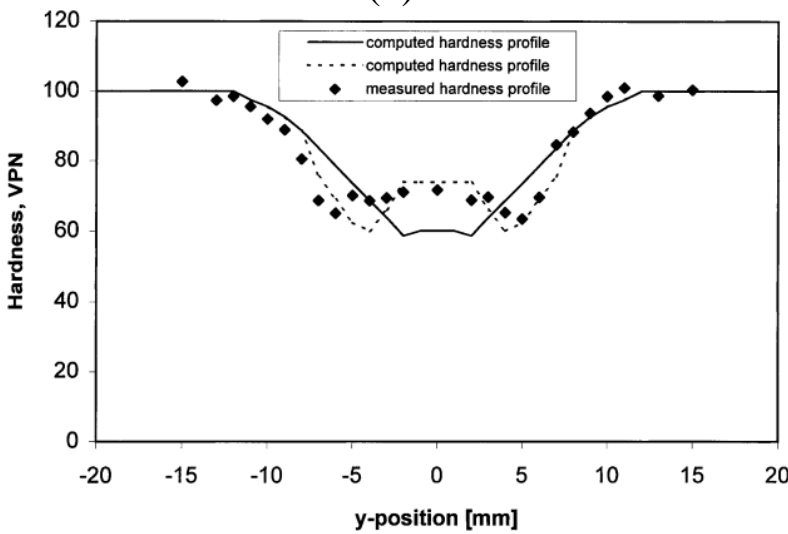

(d)

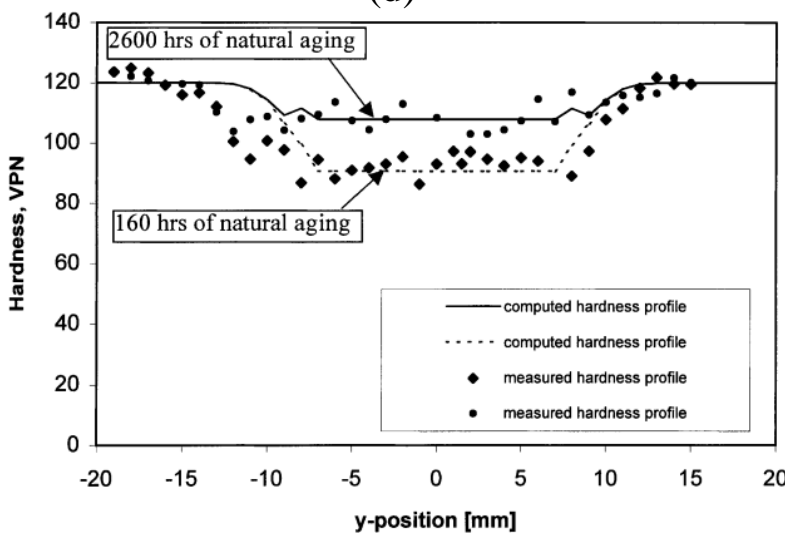

Fig. 17: Comparison between measured and predicted hardness profile in AA6082-T6 (a-b) and AA7108-T6 (c-d) aluminium alloys after FSW process. Comparisons were developed after natural ageing of $270 \mathrm{~h}(\mathrm{a}-\mathrm{b})$ or $140 \mathrm{~h}$ and $2600 \mathrm{~h}(\mathrm{c}-\mathrm{d})$. The welding were developed at low $\left(5 \mathrm{~mm} . \mathrm{s}^{-1}\right)(\mathrm{a}-\mathrm{c})$ and high $\left(12 \mathrm{~mm} . \mathrm{s}^{-1}\right)(\mathrm{b}-\mathrm{d})$ velocities for comparisons. (Frigaard et al., 2001)

Sullivan et al. (2006) developed same methodology to estimate current hardness of aluminium samples. As proposed by Myhr and Grong, dissolution of initial precipitate phase is assumed to lead to a decrease in strength and a proportional relation is also considered between volume fraction of precipitate, $f$, and hardness, $H V$. Thus, initial hardness, $H V_{\text {max }}$, correspond to the highest value and minimal value, $H V_{\min }$, is obtained in fully solutionnized condition (no hardening precipitate). The natural ageing is also integrated in modelling based on the peak temperature reached during welding as mentioned previously. Analyses of the ability of the model are developed on AA7449-T7 and AA6013-T6 aluminium alloy. For the first aluminium grade, Fig. 18 a) shows comparisons between hardness measurements and predictions developed in as-welded conditions and after ageing. 
(a)

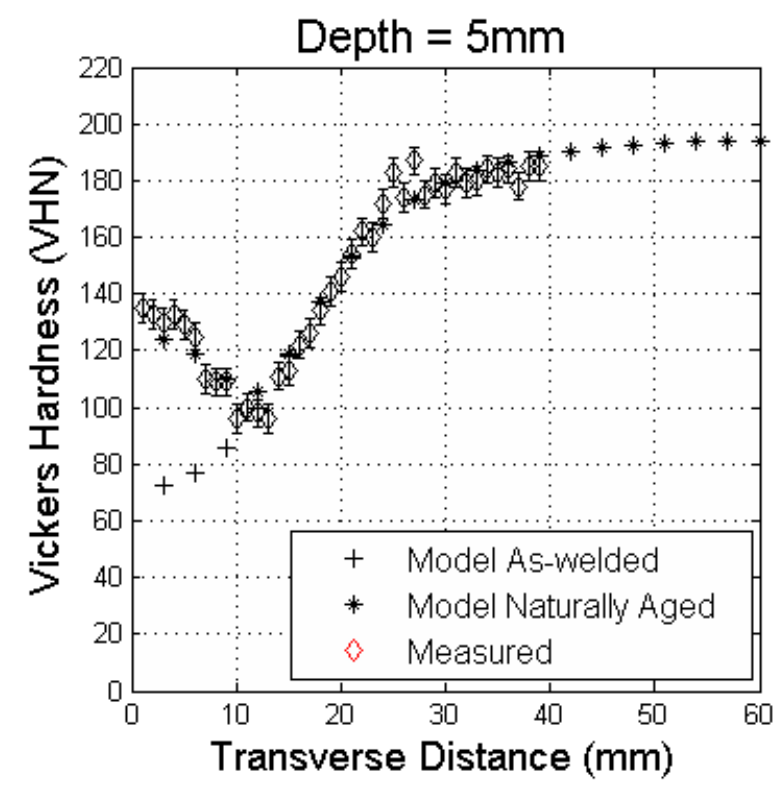

(b)

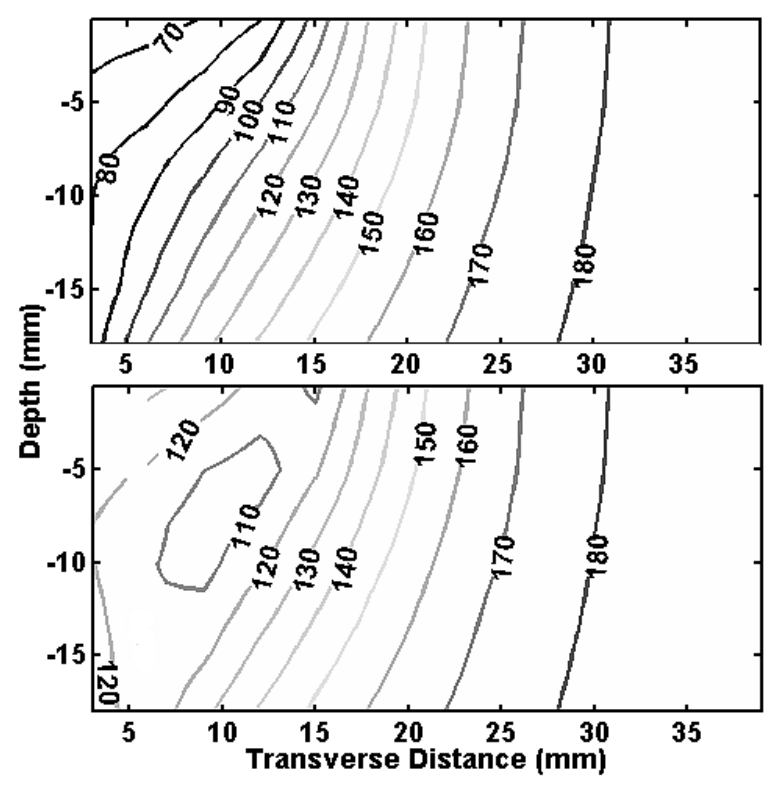

Fig. 18: Model prediction for hardness evolution in AA7449-T7 aluminium alloy (a) at a depth of $5 \mathrm{~mm}$ with comparisons between modelling (as-welded and naturally aged conditions) and measurements (b) as contour profile for simulation in as-welded condition (up) and after 1 month of natural ageing (down) (Sullivan et al., 2006).

Similar evolutions are observed between experiments and simulations. Analyses are also developed on simulations of hardness fields when considering predictions of temperature time-evolutions. After natural ageing, a minimum in hardness field is predicted at a depth of $13 \mathrm{~mm}$ from the weld centre line with a value close to $95 \mathrm{HV}$. A large extend of HAZ is also observed according to the authors.

In addition to these results, we have to point out that some improvements have been proposed in recent years to optimize the computation of the master curves and provide estimations of time evolution in precipitate fraction. More especially Lopez et al. (2008) have developed a neural networks (NN) strategy to get both the effective activation energy and associated master curves related to precipitate dissolution stage. The aim of author was to model the dissolution rate of hardening precipitates. A large range of aluminium alloy was initially investigated. Thereafter, Agelet de Saracibar et al. (2012) applied this methodology to model dissolution of precipitates during FSW processes. The aim of authors was to identify the most influential process parameters before optimization regarding quality of welded domains. A new parametrization of master curves is consequently proposed to deduce precipitate dissolution kinetics taking the volume fraction of hardening precipitates as the only state variable. Consequently, the previous equations 5-6 are modified in an optimized function considering NN strategy and applied on cylindrical precipitates. A non-linear function space representing the dissolution model is proposed and a multilayer perceptron 
with a sigmoid hidden layer and a linear output layer is used. This NN is relevant in inverse problems as corresponding to a class of universal approximators. The aim is to achieve a well fitted relation between the time logarithm $\ln \left(t / t^{*}\right)$ (i.e. input) and the complementary precipitate fraction, $1-f / f_{0}$ (i.e. output). Quite complex expressions are proposed by Lopez et al. (2008) as shown hereafter for AA-7449-T79, AA-2198-T8 and AA-6005A-T6 aluminium grades respectively where $x$ and $y$ denotes $\log \left(t / t^{*}\right)$ and $1-f / f_{0}$ quantities.

$y=0.5\left(-0.075+0.888 \tanh \left[1.429+3.671\left(\frac{x+6}{x}-1\right)\right]+1\right)$

$y=0.5\left(0.015+0.870 \tanh \left[1.037+3.535\left(\frac{x+6}{x}-1\right)\right]+1\right)$

$y=0.5\left(0.044-1.015 \tanh \left[-1.298-2.835\left(\frac{x+6}{x}-1\right)\right]+1\right)$

Investigated aluminium alloys cover a wide composition range also demonstrating the ability of optimization strategy to be applied in different dissolution rate. After this NN strategy, a good correlation is achieved when comparing optimized dissolution model with experimental observations reported in literature (Fig. 19). Results of Myhr and Grong (1991) dedicated to 6082 aluminium alloy were used in a temperature range from $200{ }^{\circ} \mathrm{C}$ to $400{ }^{\circ} \mathrm{C}$. In addition, measurements developed by Shercliff et al. (2005) on 2000 series aluminium alloy were also of interest for Lopez and used in his model validation. It should be observed that more limited temperature range has been used by Agelet de Saracibar when discussing the model application on the AA7449-T79 aluminium grade for FSW compared to the initial available experimental results. However, this strategy has been proved to successfully estimate effective activation energy as well as modelling the rate of dissolution of hardening precipitates.

(a)

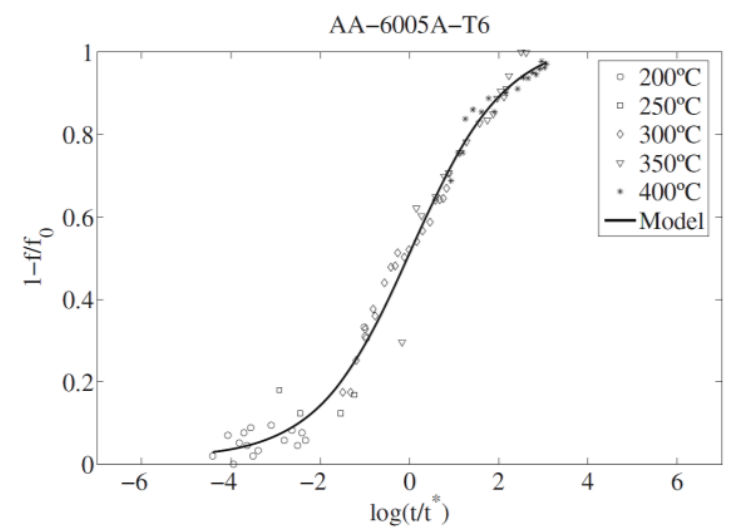

(b)

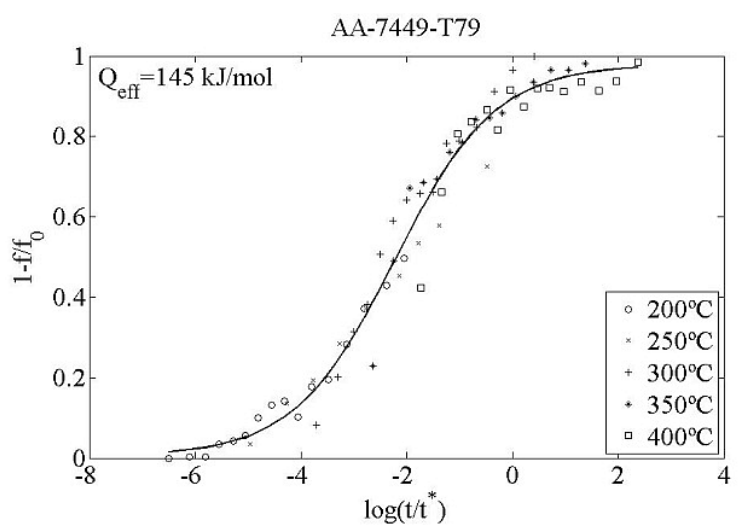

Fig. 19: Dissolution model for (a) AA6005A-T6 aluminium alloy with activation energy $Q^{*}=176 \mathrm{kj} \cdot \mathrm{mol}^{-1}$ (Lopez et al. 2008) and (b) same model applied on AA7449-T79 with activation energy $Q^{*}=145 \mathrm{kj} \cdot \mathrm{mol}^{-1}$ (Agelet de Saracibar et al. 2012). Model (continuous line) is compared with experimental data (symbols) for various temperatures. 


\section{III.2.b. Precipitate size distribution models}

\section{a. Presentation}

The Precipitate Size Distribution (PSD) model has been originally proposed by Wagner and Kampmann (1991). This model has been later developed and implemented by Myhr and Grong (2000) in finite difference approach in order to follow evolution of precipitate size distribution including the various stages of precipitation process. This approach is of main interest compared to semi-analytical models described in previous part. Indeed, these semi-analytical models do not provide a detailed description of the precipitate evolution and precipitate size distribution which is required to predict related complex phenomena or complex properties such as fracture toughness or corrosion behaviour, as mentioned previously. More realistic description of precipitate size evolution is expected also based on a relevant description of coupled physical phenomena occurring during FSW process. The numerical approach relies on the division of the precipitate size distribution into series of discrete size classes representing control volume. The numerical model consists afterwards in three components as detailed by Myhr and Grong (2000): a nucleation model to include rate of stable nuclei formation, a growth/dissolution kinetics law associated to each class and a continuity equation in order to estimate solute balance between precipitate and matrix. This approach can also be extended to model series of various types of precipitates. This model has been successfully applied afterwards to simulate precipitate size distribution evolution during heating and cooling stages in FSW processes by several authors (Table 2). Among these authors, Gallais et al. (2008) acknowledged that a large variety of models are reported in literature from Monte-Carlo model to phenomenological approach also covering various time and spatial scales. Limitations are associated to both approaches as they are not able to provide relevant information at weld scale on material enduring non-isothermal transformations. Consequently, the PSD model seems the only relevant approach able to provide useful data by integrating the whole precipitation stages endured in materials: nucleation, growth and coarsening.

Simar et al. (2007) were among the first to propose an approach dedicated to the modelling of microstructure evolution in FSW considering the influence of supersaturated solid solution. Dissolution and coarsening of fine hardening precipitates in AA6005A-T6 grades were followed during thermal cycles. Comparisons were successfully developed with experimental observations. Kamp et al. (2007) also developed PSD model including thermal and strength modelling to investigate complex precipitation mechanism developed in high strength 7449 aluminium alloys during FSW. In their opinion, microstructure evolutions are highly influenced by peak temperature also considering heating and cooling rates. Final precipitate densities were used afterwards to determine material strength and developed comparisons to hardness estimation. More recently, Legrand et al. (2015b) have developed a PSD model for multicomponent alloy coupled with thermodynamic database also including 
unsteady growth laws. This model has been applied on AA2024 grade to investigate evolution of hardening precipitates in aluminium alloys dedicated to aircraft industries. Dos Santos et al. (2018) developed similarly PSD model dedicated to AA7449 aluminium grades also including recrystallization mechanisms. This model has been embedded in a process model based on a CFD (computational fluid dynamics) framework developed in FLUENT (2019). The evolutions of three distinct precipitate populations are followed. More interestingly, the influence of grain refinement on precipitations mechanisms is taking into account in a coupled approach between grains and precipitates evolutions. These various applications cover a wide range of aluminium grades and choice of process parameters. This PSD method consequently mimics the size distribution of precipitates (Fig. 20) and its temporal evolution. A detailed description of this model is provided hereafter in order to highlight the main steps of PSD approaches.

(a)

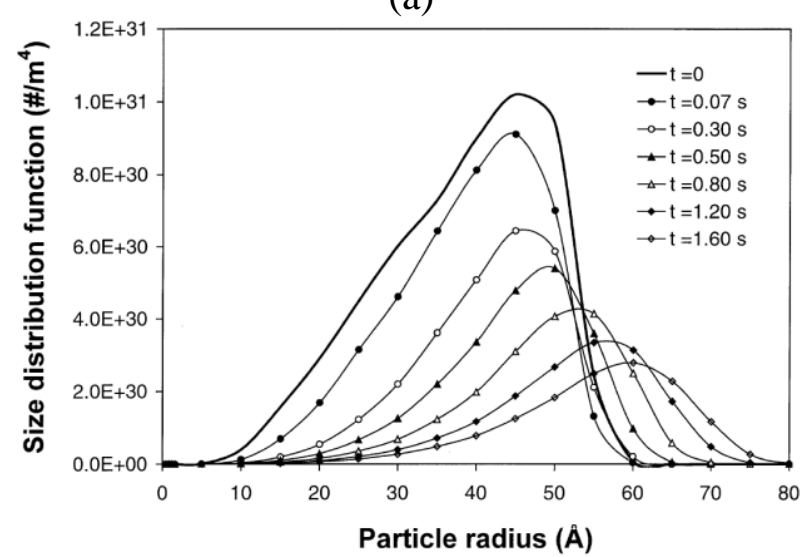

(b)

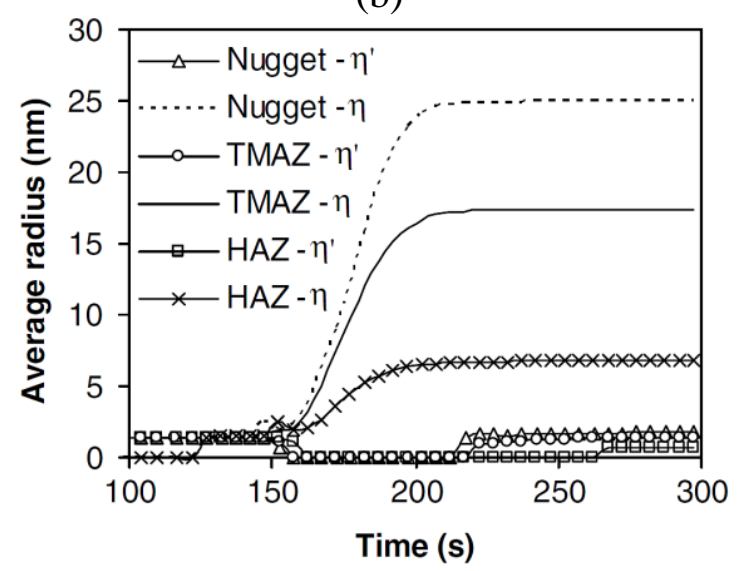

Fig. 20: (a) Precipitate size distribution evolution in isothermal heating $\left(T=380{ }^{\circ} \mathrm{C}\right.$ on an $\mathrm{Al}-$ Mg-Si alloy) (Myhr and Grong (2000)). Points correspond to the position of simulated classes. (b) Average radius evolution for two precipitate phases ( $\eta$ and $\eta$ ') in various welded domains on a 7449 aluminium alloy predicted with same PSD approach (Kamp et al., 2006).

\section{b. Methodology}

The local conservation equation of precipitate density, $N^{\varphi}$, associated to each precipitate phase $\varphi$, is usually given with the classical formulation proposed by Myhr and Grong (2000).

$\frac{\partial N^{\varphi}}{\partial t}=-\frac{\partial\left(N^{\varphi} v\right)}{\partial r}+J^{\varphi}$

where $v$ is the growth velocity associated to precipitates radius $r$. The variable $J$ represents the nucleation rate of precipitates with specific radius $r$. This equation is usually discretized by finite differences considering the regular grid of class size, also including a 'streamlineupwind/Petrov-Galerkin' (SUPG) formulation, for numerical stability reasons. Two mains parameters are still to determine, the nucleation rate and the growth velocity.

- Nucleation rate 
The nucleation rate, $J,\left[\mathrm{~m}^{-3} \cdot \mathrm{s}^{-1}\right]$ corresponds to the number of stable precipitate nuclei forms per unit volume and unit time. Classical general nucleation theories dedicated to the modelling of precipitation in metallic alloys as the one described by Perez et al. (2008) report systematically the use of same variable. All the nucleation is considered as occurring at the single critical radius, $r^{*}$, although this value may largely evolves during the entire process depending from matrix composition and local temperature. Russel (1970) proposes an extended formulation written in the classical expression:

$J=Z \beta N_{0} e^{-\frac{\Delta G^{*}}{R T}} e^{-\frac{\tau}{t}}$

with $Z$ the Zeldovich factor, $\beta$, the atomic impingement rate, $\tau$ the incubation time, $N_{0}$ the number of nucleation sites still available and $\Delta G^{*}$ the activation energy for nucleation. This latter value corresponds to the required energy to promote nucleation of precipitates and is assumed as the Gibbs free energy between matrix and precipitate. The incubation time, $\tau$, is usually neglected except by Kamp et al. (2007). The number of available nucleation sites is the difference between the initial number of nucleation sites, $N_{0}^{0}$, and the number of the ones activated at current time, $t, N_{0}^{t}$. The Zeldovich factor has been introduced to consider precipitate size fluctuation around the critical radius, $r^{*}$, and possibility of self-dissolution. Zeldovich (1943) proposed the following expression (Eq. 13) for this factor.

$Z=\sqrt{\frac{-1}{2 \pi N_{a v} k_{B} T}\left(\frac{\partial^{2} \Delta G^{*}}{\partial n^{2} \mid n^{*}}\right)}$

where $N_{a v}$ and $k_{B}$ are respectively the Avogadro number and Boltzmann constant. The value $n$ corresponds to the number of atoms in the nuclei. This formula can also be simply expressed as:

$Z=\frac{V_{m}^{p}\left(\Delta G_{v}^{p}\right)^{2}}{8 \pi\left(\gamma^{m / p}\right)^{3 / 2} N_{a v} \sqrt{k_{B} T f(\theta)}}$

where $V_{m}^{p}$ is the molar volume of precipitate, $\gamma^{m / p}$ is the interfacial energy between matrix (m) and precipitate (p) and $\Delta G_{v}^{p}$ is the volume free energy associated to phase transformation from matrix to precipitate. According to Gallais et al. (2008), Eq. (13) may lead to a value of the order of $1 / 20$ to $1 / 40$ for $Z$ coefficient. Simar et al. (2012) used the largest estimation as an exact value in the modelling of precipitation phenomena in FSW processes.

The atomic impingement rate, $\beta$, (Eq. 12) corresponds to the probability of atoms attachment onto the surface of precipitate. This parameter generally relies on the atom of lowest diffusivity. Consequently, Simar et al. (2007) used the concentration of Mg element in its estimation when investigating precipitation of the Mg2Si phase on AA6005 alloy. Perez et al. (2008) rightly points the large number of possible expressions reported in the literature 
without clear justification and promotes the analytical expression of Russell (1970). In multicomponent alloy, an extended general formulation is proposed by Legrand (2015a) as:

$\beta=\frac{4 \pi r^{* 2}}{a^{4}} \operatorname{Min}_{\{1 \leq i \leq N\}} D_{i}^{m} X_{i}^{m}$

where $a$ is the lattice parameter of matrix phase. $D_{i}^{m}$ and $X_{i}^{m}$ are respectively the diffusion coefficient and composition of element $i$ in the matrix phase. These latter values depend both from local temperature and composition. Svoboda et al. (2004) has also proposed some better expressions including diffusion and composition effect of all alloying elements.

The Gibbs free energy associated to nucleation, $\Delta G^{*}$, has still to be determined, also considering curvature effect and heterogeneous nucleation. Simar et al. (2012) expressed this value considering the interfacial energy, $\gamma^{m / p}$, in addition with the volume enthalpy free energy, $\Delta G_{v}^{p}$, and the strain energy change, $\Delta G_{e}^{p}$, associated to precipitation:

$\Delta G^{*}=\frac{16 \pi}{3} \frac{\gamma^{m / p^{3}} f(\theta)}{\left(\Delta G_{v}^{p}+\Delta G_{e}^{p}\right)^{2}}$

The function $f(\theta)$ is the capillary function associated to the wetting angle when heterogeneous nucleation is assumed, as an example, on dispersoids and dislocations (Gallais et al., 2008). Bardel et al. (2014) also proposed corrected expressions when considering nonspherical geometries. The strain energy change, $\Delta G_{e}^{p}$, can be computed from elastic properties and density evolution associated to both matrix and precipitates. The estimation of the volume Gibbs free energy, $\Delta G_{v}^{p}$, seems more complex. It corresponds to the Gibbs free energy variation between matrix phase and precipitate phase considering same compositions. This value is usually computed considering an ideal solution hypotheses as proposed by several authors (Eq. 17). Simar et al. (2012) provided details on this expression also considering composition effect in each element:

$\Delta G_{v}^{p}=\frac{R_{g} T}{V_{m}^{p}} \sum_{i=1}^{N} X_{i}^{p} \cdot \ln \left(\frac{X_{i}^{m}}{X_{i, e q}^{m}}\right)$

where the summation is developed on all elements (including main element). $X_{i}^{p}$ is the precipitate composition at the equilibrium, $X_{i}^{m}$ is the initial matrix composition and $X_{i, e q}^{m}$ is the matrix composition at the equilibrium after phase change (i.e. precipitation). It should also be pointed out that this value can be directly computed based on thermodynamic properties and associated databases for matrix and precipitate phases. This approach is introduced in the TC-PRISMA software (TC-PRISMA, 2019) and similarly applied by Serrière et al. (2002) to model precipitate evolutions. In addition, the estimation of Gibbs free energy and strain energy change lead to the computation of the critical nucleation radius, $r^{*}$, as:

$r^{*}=-\frac{2 \gamma^{m / p}}{\Delta \mathrm{G}_{v}^{p}+\Delta G_{e}^{p}}$ 
In the precipitate distribution models, Gallais et al. (2008) considered that precipitates nucleate in the single class containing the critical nucleation radius. Legrand (2015a) proposed to share precipitate nucleation between this latter and the next class following the approach previously implemented by Serrière et al. (2002). This choice consequently depends from the class definition and spatial position. The nucleation rate is then applied considering the estimated value at the current time (Eq. 12)

\section{○ Growth velocity}

The growth velocity has to be determined in each precipitate class depending from the precipitate size and composition. This stage includes both growing and dissolution regimes induced by curvature effect. Indeed, precipitate composition at the interface with matrix evolves depending from the curvature radius and the associated increase of the Gibbs free energy as illustrated on Fig. 21 (Gibbs Thomson effect). Largest precipitates develop $\left(v_{2}>0\right)$ while smallest precipitates dissolve $\left(v_{1}<0\right)$ due to the position of interfacial compositions, $X_{i, e q}^{m / p}$, in the matrix compared to the average compositions, $X_{i}^{m}$. These phenomena explained the coarsening stage currently observed at longer time when only largest precipitates are still observed leading to hardness decrease. However this phenomenon also illustrates the need to develop growth model including Gibbs Thomson effect.

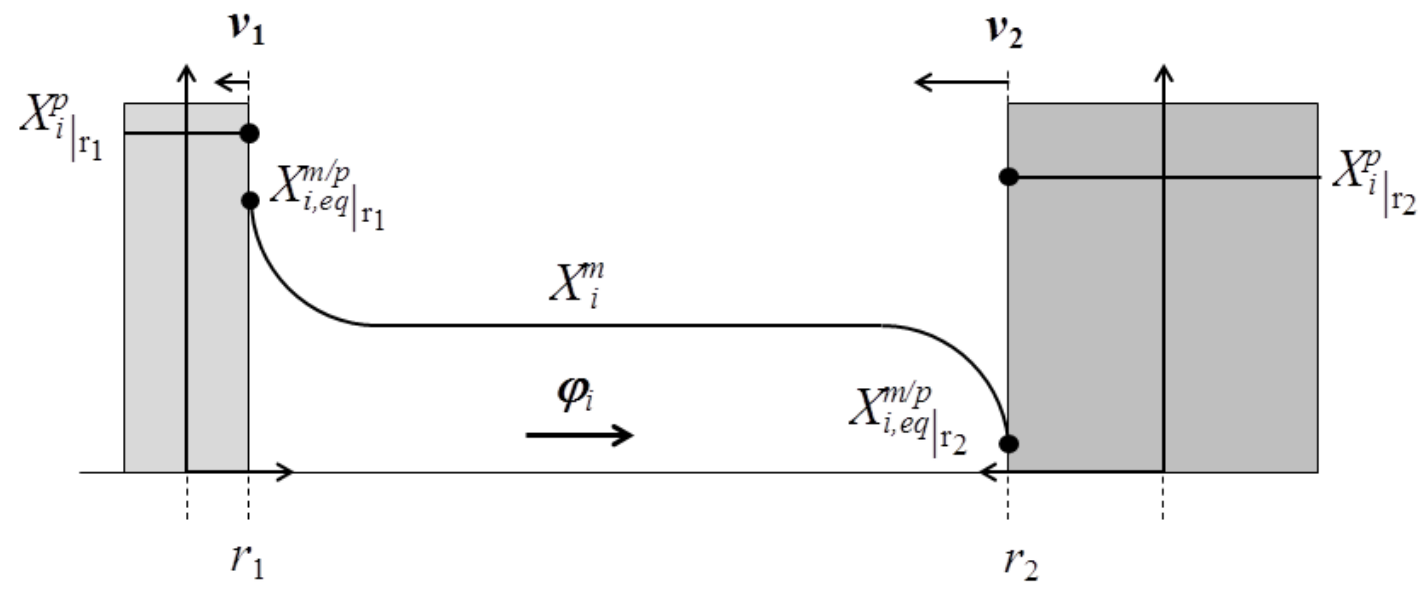

Fig. 21: Dependence of the precipitate growth kinetics from the curvature radius $\left(r_{2}>r_{1}\right)$. Evolutions are presented when precipitates are enriched compared to the matrix. Solute flux, $\boldsymbol{\varphi}_{\boldsymbol{i}}$, illustrates the direction of flow in element $i$ from the smallest to the largest precipitates.

The growth velocity is usually computed considering the volume and interfacial conservation equations. These latter are solved with a far field composition at large distance from precipitate/matrix interface equal to the average matrix composition, $X_{i}^{m}$ for any element $i$.

In the Laplace regime, the time evolution in the composition field is neglected leading to a simplified expression for the solute conservation equation as highlighted by Aaron et al. (1970). The growth velocity is thereafter expressed as: 
$v=\frac{D_{i}^{m}}{r} \Omega_{i}=\frac{D_{i}^{m}}{r} \frac{X_{i}^{m}-X_{i}^{m / p}}{X_{i}^{p}-X_{i}^{m / p}}$

where $D_{i}^{m}$ is the diffusion coefficient of element $i$ in the matrix phase considered as homogeneous and $\Omega_{i}$ is the oversaturation associated to same element. However this formula is restricted to spherical geometry and no-extension to cylindrical or plate geometries is available, even if other approaches are conceivable for such geometries.

Following the original model of Myhr and Grong (2000), this growth velocity expression has consequently been largely used in the literature. In their review article, Simar et al. (2007) reported similar expression to describe dissolution or coarsening rate associated to precipitates when applying PSD models assuming that growth is only controlled by diffusion processes. Authors have seldom reported approaches associated to other geometries. However, Gallais et al. (2008) have adapted their size class model to the complexity of precipitate geometries encountered in AA6056 aluminium grade as observed in TEM. Indeed, observations reported elongated precipitates with size factor close to 25 . Consequently, the growth rate in the lateral direction of cylindrical precipitates is adapted from the one associated to spherical shapes. More interestingly, Kamp et al. (2006) applied their model with both spherical shaped and plate shaped precipitates. For this latter case, a Zeener-type model is assumed where precipitates are considered as discs with hemispherical borders. Limited effects were reported on final results despite a significant increase in model complexity.

In addition, it should be noticed that Eq. (19) does not consider cross-diffusion effect. An improvement of the Laplace approximation dedicated to multicomponent alloys has been recently proposed by Legrand (2015a) corresponding to the extension of the exact solution developed by Aaron et al. (1970). This approach follows the work of Guillemot and Gandin (2017) and is based on the exact solution in the unsteady growth regime when temporal evolutions of the solute field are considered. This approach is also useful for large oversaturation (i.e. close to 1) when the Laplace approximation is irrelevant. In such conditions, the growth velocity is also expressed depending from the oversaturation as:

$\Omega_{i}=\frac{x_{i}}{2}\left(1-\frac{\sqrt{\pi}}{2} \sqrt{x_{i}} \exp \left(\frac{x_{i}}{4}\right) \operatorname{erfc}\left(\frac{\sqrt{x_{i}}}{2}\right)\right) \quad$ where $x=\frac{2 r v}{D_{i}}$

As noticed by Kamp in multicomponent alloys, all the equations linking oversaturation and growth velocity (Eq. 19, 20) should lead to the same solution for the velocity value $v$ when applied to any added elements. Consequently, a single solution should be computed for all the equations.

The Gibbs Thomson effect is afterwards currently considered in the expression of the solubility product, $K(r)$, as proposed by Nicolas and Deschamps (2003):

$K(r)=K_{0} \times \exp \left(\frac{2}{r} \frac{\gamma V_{m}}{R_{g} T}\right)=\prod_{i=1}^{N} X_{i}^{m / p^{X}} \times \exp \left(\frac{2}{r} \frac{\gamma V_{m}}{R_{g} T}\right)$ 
where $K_{0}$ is the solubility product without curvature effect and $V_{m}$ is the molar volume of precipitate. This solubility product expression is validated for high dilution when the activity of elements is close to its concentration. As proposed by Kamp et al. (2006), $K_{0}$ is computable considering the thermodynamic database software JMatPro (2019). Indeed, this software based on the CALPHAD (2019) methodology provides data on equilibrium compositions in the matrix and precipitate phases, $X_{i}^{m / p}$ and $X_{i}^{p}$ (Eq. 21). Similarly, Dos Santos et al. (2018) also deduced thermodynamic properties of all phases encountered in precipitation processes from the same tool. Legrand et al. (2015b) develop similar estimations however based on Thermocalc (2019) software database. The system is composed from the $N-1$ equations (19), the $N-1$ tie-line definitions and the equation (21). This system has to be solved for any radius $r$. This solution gives thereafter access to the precipitate and matrix/precipitate compositions $\left\{X_{i}^{m / p}, X_{i}^{p}\right\}_{1 \leq i \leq N}$ as well as to the current growth velocity, $v$, for a given class radius, $r$. The equation (11) is then solved in a time and space-discretized approach in a SUPG formulation.

\section{c. Results}

Several results reported in the literature demonstrate the efficiency of PSD modelling to follow precipitate distribution. Some relevant simulations are described hereafter corresponding to results reported in the literature and showing the clear interest of this approach. One of the first results in PSD approach to model precipitate evolution in FSW process was provided by Gallais et al. (2008) when investigating precipitate evolution in AA6056 aluminium alloy. The alloy was considered as a quaternary aluminium alloy (Al-Mg$\mathrm{Si}-\mathrm{Cu}$ ) where complex precipitation sequences are reported in literature. The complex precipitation steps are carefully described also considering TEM observations providing the microstructure evolutions in SZ, TMAZ and HAZ. The precipitation sequence should be considered as solid solution $\rightarrow G P \rightarrow \beta^{\prime \prime} \rightarrow \beta^{\prime}+Q \rightarrow Q+\beta$. However, Gallais and coauthors made no distinction between $\beta^{\prime \prime}, \beta^{\prime}$ and $Q$ phases and consider precipitation as corresponding to the evolution of the single $Q$ phase in a simplified approach. The PSD model is therefore applied to investigate precipitate evolution with this single type of precipitate through the whole process.

Two different aluminium grades are investigated in the AA6056 series. The first one, T4, corresponds to a natural ageing of an aluminium grade after solubilisation and water quenching. The second one, T78, corresponds to the development of precipitate during heat treatment after solubilisation and quenching $\left(6 \mathrm{~h} / 175^{\circ} \mathrm{C}\right.$ and $\left.5 \mathrm{~h} / 210^{\circ} \mathrm{C}\right)$. In order to take into account the various nucleation sites and associated precipitation kinetics observed by TEM, Gallais considers three distinct distributions associated respectively to the homogeneous precipitation inside grains and to two other different heterogeneous precipitation mechanisms associated to dispersoids and dislocations. The distinction between classes is made considering specific nucleation law for each type of precipitation site with specific free energy for nucleation, $\Delta G^{*}$, and critical radius, $r^{*}$ : 
$\Delta G^{*}=\frac{224 \pi \alpha}{81} \frac{\gamma^{m / p^{3}} f(\theta)}{\left(\Delta G_{v}^{p}+\Delta G_{e}^{p}\right)^{2}}$

$r^{*}=-\frac{4}{3} \frac{1+\alpha}{\alpha} \frac{2 \gamma}{\Delta \mathrm{G}_{v}^{p}+\Delta G_{e}^{p}}$

where $\alpha$ is the shape factor in each precipitate class. These two equations respectively replace the previous equation (16) and (18) and help to enhance the modelling of nucleation processes in FSW. The nucleation site density for the three types of precipitate is provided from density of atoms (homogeneous), experimental TEM observations (dispersoids) and considerations on a stretching of the material (dislocation/strain) around $10 \%$. Considering the full precipitation process, Gallais also mentioned that post-weld natural ageing possibly induces an increase of the yield strength due to GP zones formation. However, these GP zones are metastable phases and still difficult to model consequently. The origin of their effect on hardening is also unclear. However, a final volume fraction of GP zones is assumed as proportional to the solid solution concentration minus a miscibility gap. Their effects on hardness are also based on classical contribution law. The temperature history is provided with the FlexPDETM software with input parameters corresponding to the dimensions of workpiece and tool diameter in addition with material data. Boundary conditions are then applied with natural convection on the top surface and heat conduction on the bottom part. Heat in introduced in the process through a welding efficiency factor, $\eta,(\sim 60 \%)$ multiplying the average mechanical power input, $P$. One the main interest of this approach is the partitioning proposed by authors between shoulder $(\sim 80 \%)$ and pin $(\sim 20 \%)$ for local heat introduction after experimental measurements.

A specific approach is also proposed by Gallais for experimental validation of PSD models. Indeed, the experimental estimation of precipitate fraction in FSW joint and their dependence from distance from weld centreline is based on Differential Scanning Calorimetry (DSC) analysis. Similar innovative approach was also previously proposed by Genevois (2004). On each collected sample in FSW joint, the area, $B$, between DSC curves and baseline for each precipitation sequence is estimated. When comparing this area to the one associated to the base material without precipitation sequence, $B_{0}$, the relative fraction of precipitate, $f_{Q}$, is simply provided by the comparison between area:

$f_{Q}=1-\frac{B}{B_{0}}$

The evolution in precipitate fraction can be reported depending from sample positions as shown on Fig. 22 for both T4 and T78 AA6056 aluminium grades. Tool rotational velocity and welding speed were respectively equal to $1100 \mathrm{rpm}$ and $1100 \mathrm{~mm} \cdot \mathrm{min}^{-1}$. Comparison between simulation and DSC measurements demonstrates the efficiency of the PSD model to follow precipitate evolution in each class. Indeed, experimental measurements are close to simulations when considering only a specific type of precipitation mechanism. Gallais also 
concludes that PSD model presents some slightly overestimation of the total volume fraction of precipitates but predict correctly evolutions in various domains (SZ, TMAZ, HAZ). Moreover, the mean radius predicted in the low hardness zone $(\sim 80 \mathrm{HV})$ is of the order of 7.7 $\mathrm{nm}$ which is close to the TEM observations where a value of $7.3 \mathrm{~nm}$ was reported (T78 grade). The model also demonstrates different evolution between T4 and T78 conditions. Considering T4 aluminium grade, heterogeneous precipitation occurs on the dispersoids at small distance from the welding line and on dislocations at larger distance. Dissolution of precipitates is then also observed further away from welding line. However, on T78 grade, heterogeneous precipitation process is observed at same distance with a stable volume fraction of precipitate of $2 \%$. Differences in precipitation processes are also investigated by Gallais at the same distance $(\sim 7.5 \mathrm{~mm})$ corresponding to the lowest hardness for both experiments. Heterogeneous precipitation is observed in simulations on both dislocations and dispersoids on T4 grade without any homogenous precipitation. On the opposite, homogeneous precipitates are observed on T78 grade with both dissolution and growing stage during welding. These evolutions lead to large difference in final state on precipitate distribution. It should be mentioned that some discrepancies between experimental observations and modelling where also pointed out by Gallais in previous isothermal simulation also developed with PSD models. The main limitation of such approach which only considers $Q$ phase may explains such differences whereas both formation of GP zones and precipitation of $\beta^{\prime \prime}$ phase also occur in the same temperature range.

(a)

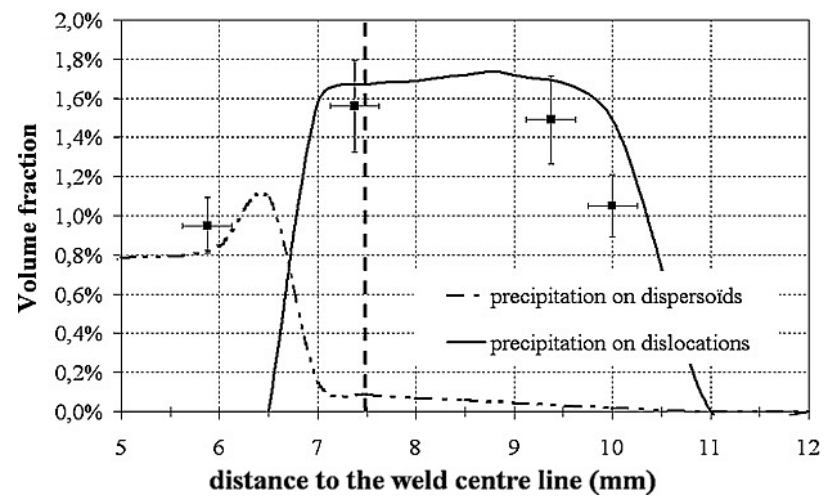

(b)

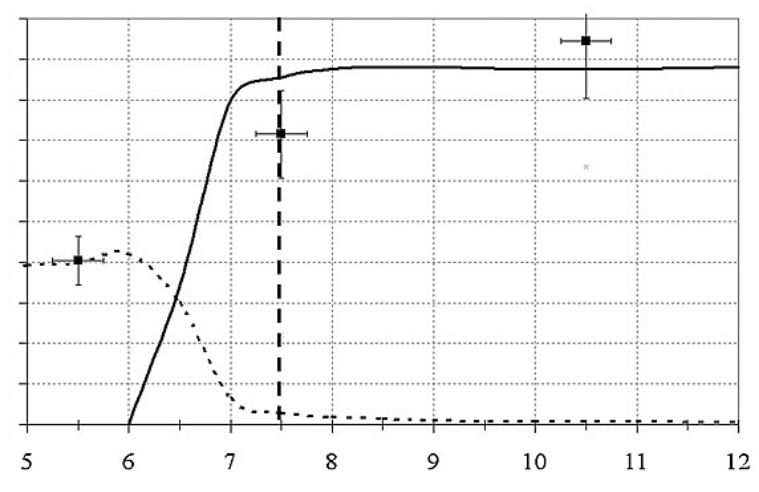

Fig. 22: Evolution of precipitate volume fraction in the TMAZ and HAZ zones in the FSW joint on (a) AA6056 T4 and (b) AA6056 T78 aluminium grades. Experimental values correspond to the total volume fraction. (Gallais et al., 2008)

A second application recently reported in literature on the use of PSD approaches is the activity developed by Legrand et al. (2015a). This latter was part of a wider research project dedicated to the mastering and control of FSW processes for its application and diffusion in aeronautical industries as an alternative solution to riveting processes. Consequently this project focuses its interest on AA2024 (Al-Cu-Mg) aluminium grades used for panel manufacturing in aircraft fuselage. The precipitation sequence of this alloy was also subject to 
many discussions especially on co-cluster and GPB zones formation. Following the assumptions of Genevois et al. (2005), the precipitation sequence was described as solid solution $\rightarrow$ GPB $\rightarrow$ S phase. Consequently, the PSD model is applied simultaneously on both GPB and S-phase precipitates considering their interaction with the single aluminium (FCC) matrix. Only GPB zones are assumed in the material in delivery state before process. In addition the effect of observed intermetallic phases is not considered in this model and simulations were developed on compositions experimentally measured by EDS analysis at large distance from these intermetallic domains. Thermodynamic coupling is one of the interests of the model developed by Legrand. This latter is based on the scientific software Thermo-Calc (2019) and associated TCAL3 database on aluminium alloy (TCAL3, 2014) in order to estimate precipitate / matrix interfacial compositions and associated growing kinetics. Consequently, the $X_{i}^{p}$ and $X_{i}^{m / p}$ compositions (Eq. 19) are estimated from thermodynamic computations also considering curvature effect as well as the Gibbs free energy associated to nucleation, $\Delta G^{*}$, (Eq. 12) for any current temperature, $T$. The growing kinetics is based on Aaron et al. (1970) solution extended to multicomponent alloys by Guillemot and Gandin (2017) as detailed in previous part (Eq. 20).

Legrand has also developed a careful DSC analysis on a large set of samples to calibrate the PSD model. DSC measurements were developed on samples in initial state providing access to nucleation parameters for the two set of precipitate classes: the interfacial energy, $\gamma^{m / p}$, the wetting angle, $\theta$, (Eq. 16) and the initial number of nucleation sites, $N_{0}^{0}$. As a second step, DSC analyses were conducted on heat treated samples at $190{ }^{\circ} \mathrm{C}$ during various durations (Fig. 23 a). These treatments induce both dissolution of GPB zones and precipitation of S-phases. Area between curves and baselines in DSC experiments are analysed similarly as Gallais et al. (2008) for an estimation of fraction of both GPB zones and S-phases after heat treatment. These experiments provide direct validation of the PSD model when comparing time evolution of both fractions of precipitates between experiments and simulations (Fig. 23 b). Dissolution of the GPB zones and growth of the S-phase are correctly predicted with similar time profile evolutions even if some discrepancies are observed. In addition, hardness measurements were performed on samples which provide calibrating parameters between precipitate size distribution and mechanical properties in AA2024 aluminium alloys. 
(a)

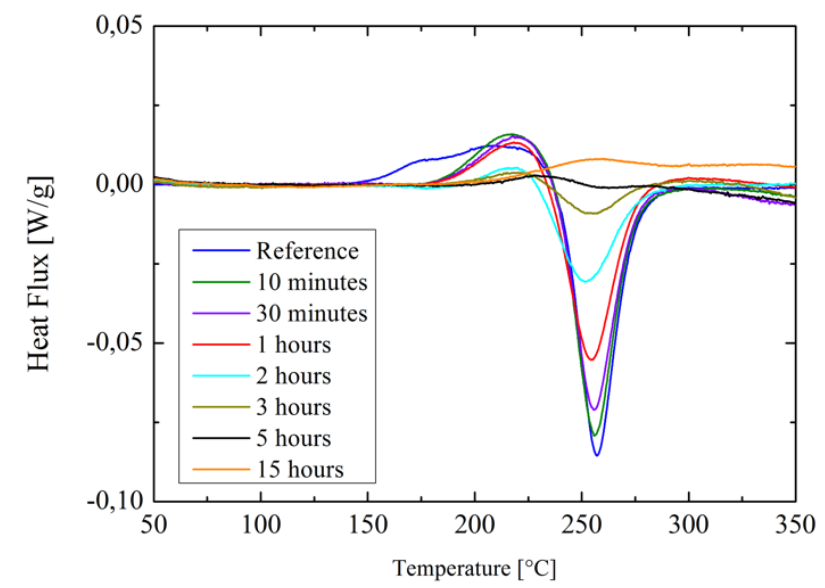

(b)

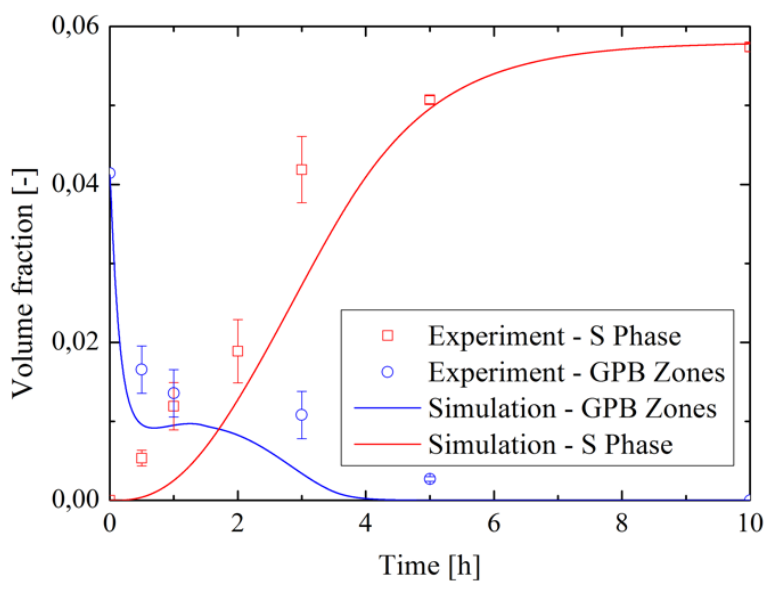

Fig. 23: (a) DSC curves for the determination of relative phase fractions associated to AA2024 material in delivery state (Reference) and after specific heat treatment durations (10 min to 15 hours) at $T=190^{\circ} \mathrm{C}$ (slope $\sim 10^{\circ} \mathrm{C} \cdot \mathrm{min}^{-1}$ ). (b) Evolution of precipitate phase fraction for same samples depending from heat treatment duration deduced from DSC curves analysis for S phase (red symbols) and GPB zones (blue symbols) - Comparison with simulations for same S-phase (red line) and GPB zones (blue line) (Legrand, 2015a).

The model has been afterwards applied to the simulation of precipitate evolution during FSW processes assuming that only GPB zones are present in initial state with an associated fraction based on DSC measurements. Temperature evolutions were provided from thermo-mechanical simulation developed at macro-scale by Gastebois (2015) on the entire pieces. An Arbitrary Lagrangian-Eulerian approach (ALE) based on a viscoplastic (i.e. Norton-Hoff) constitutive model has been developed with this aim as detailed by Fourment (2016). These temperature evolutions computed at various distances from the weld centre line were introduced as input data in PSD simulations. Fig. 24 a-b) shows the profile of final relative fraction in HAZ, TMAZ and SZ (Nugget) domains for both S phase and GPB zones for a tool rotational velocity of $1200 \mathrm{rpm}$ and an advance velocity of $1 \mathrm{~mm} \cdot \mathrm{s}^{-1}$. Evolutions of precipitate fractions were also estimated from samples cut in a cross section of welded pieces using the same DSC curves analysis. Similar fractions evolutions are observed between simulations and experiments in S-phase. S-phase precipitates are absent in the non-affected domain and begin their development at the boundary between HAZ and TMAZ. A large fraction of small precipitates $(\sim 6 \mathrm{~nm})$ develops leading to an increase in hardness also observed experimentally by Legrand (2015a) (Fig. 11 b). At the same position (Fig. 24 b), a complete dissolution of GPB zones is observed and the hardness is mainly due to S-phase precipitate. The volume fraction of S-phase precipitate then decreases with an increase in the precipitates size $(\sim 45 \mathrm{~nm})$. The set of coarse precipitates induces a low hardness value and a large decrease in mechanical properties. In the nugget domain, a low fraction of S-phase is also predicted with large precipitate size. The complete dissolution of GPB zones is still observed without any expected re-precipitation (Fig. 24 a) as natural ageing at ambient 
temperature is not assumed in present simulations. This softening of the nugget domain just after FSW process is expected as GPB zones are dissolved by FSW processing. However these GPB zones should be later developed in the matrix over a long period of time. Consequently, some discrepancies are observed in the central part of the welded domains between PSD model and DSC measurements conducted on natural-aged samples. However, the general trends in precipitates fraction evolution are well reproduced before natural ageing and well-suited predictions are obtained when considering thermodynamic coupling.

(a)

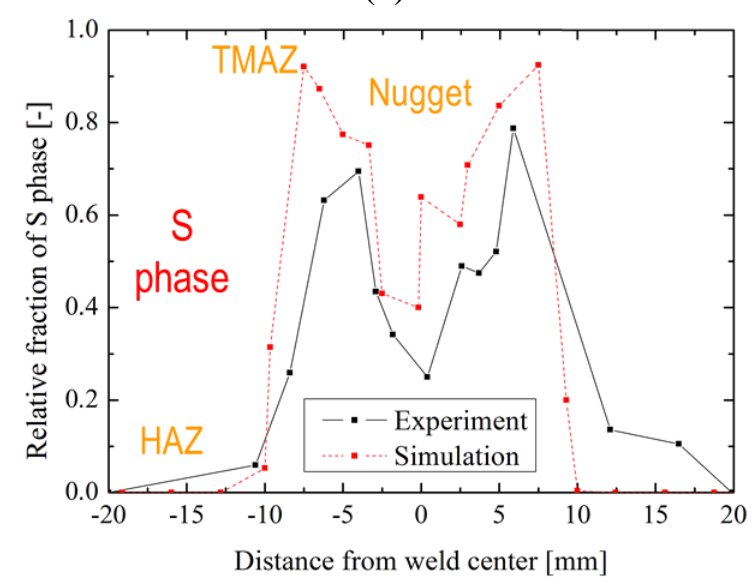

(b)

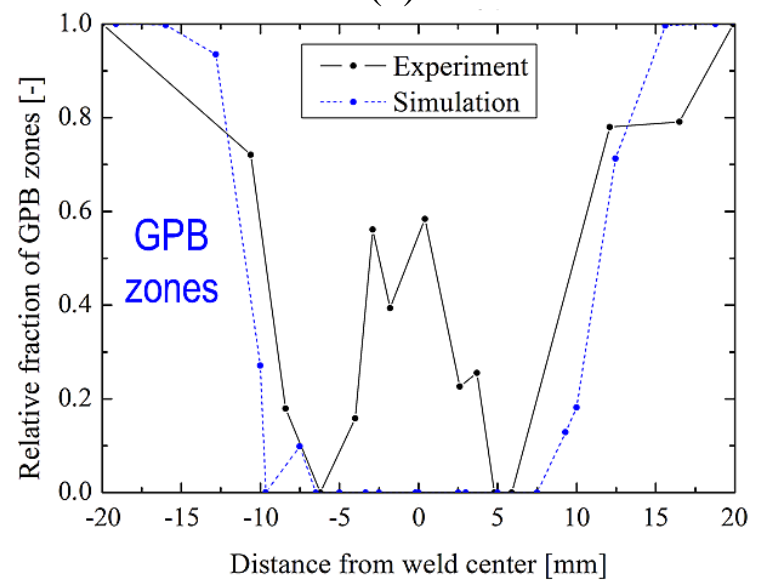

Fig. 24: Relative fraction of (a) S phase and (b) GPB zones as a function of the distance from weld centreline. Comparison between experiments (black plain line) and simulation (coloured dashed line) (Legrand, 2015a).

One of the most recent applications in PSD approach has been provided by Dos Santos et al. (2018) when applying the PSD model to follow precipitate evolution in 7449-TAF aluminium alloys. The approach is also interesting as it combines models that both consider the effect of thermal and deformation cycle on precipitate and grain structure evolution. Consequently the influence of Geometric Dynamic Recrystallization (GDRX) in SZ is also included in precipitate nucleation. The effect of grain refinement is then investigated with initial grain size controlled by GDRX phenomenon in SZ as proposed by Robson et al. (2010). Kamp et al. (2006) provide a detailed description of their application of PSD model with precise presentations of the main resolution steps. The FLUENT software (2019) is used in order to compute temperature field evolution induced by stirring process considering the alloy as a large-viscosity fluid. Mechanical properties depend from temperature and strain rate. The PSD model considers consequently the output of macro-scale simulation (temperature, strain and strain rate) to estimate evolution in precipitate size distribution. Distinct precipitate populations are separately tracked: the grain interior metastable, $\eta^{\prime}$, the grain interior equilibrium, $\eta$, and the grain boundary $\eta$. At initial state, the microstructure is mainly composed of $\eta^{\prime}$ precipitates. Simulations provide direct access to precipitate evolution for each precipitate type. Fig. 25 shows the precipitate size evolution of the three classes depending from weld centreline distance. Complex sequences of microstructural evolution are 
observed also including complete dissolution of $\eta^{\prime}$ precipitates in the early stages when temperature increases. All the $\eta^{\prime}$ may dissolve or form stable $\eta$ precipitate depending of their size. During continued heating, the $\eta$ precipitates become unstable and begin a dissolution process just before the temperature peak. When the matrix is supersaturated in the age hardening added elements as zinc, magnesium or copper, a reprecipitatation mechanism occurs during cooling. This mechanism is also favoured for $\mathrm{GB}-\eta$ precipitates at position close to the pin (Fig. $25 \mathrm{a}-\mathrm{b}$ ). This latter is due to the grain recrystallization mechanism and to the large area of grain boundaries promoting heterogeneous nucleation. In addition, the authors show a distance effect in their result when comparing the final state depending from the distance to the weld centreline.

(a)

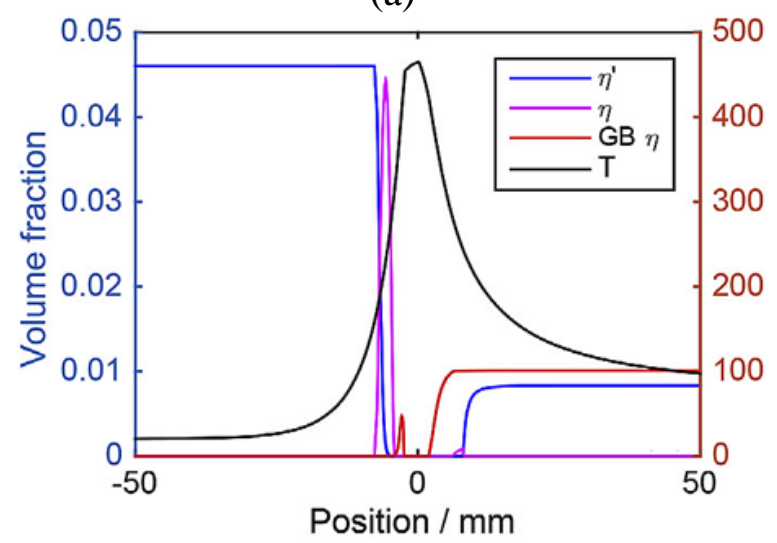

(c)

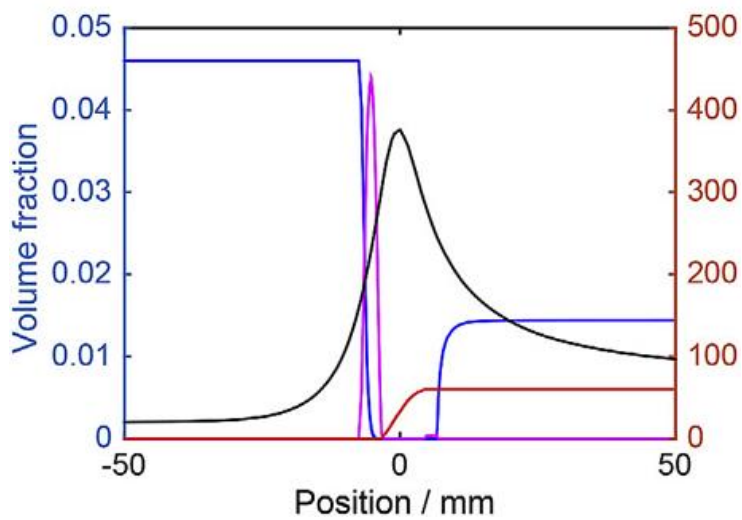

(b)

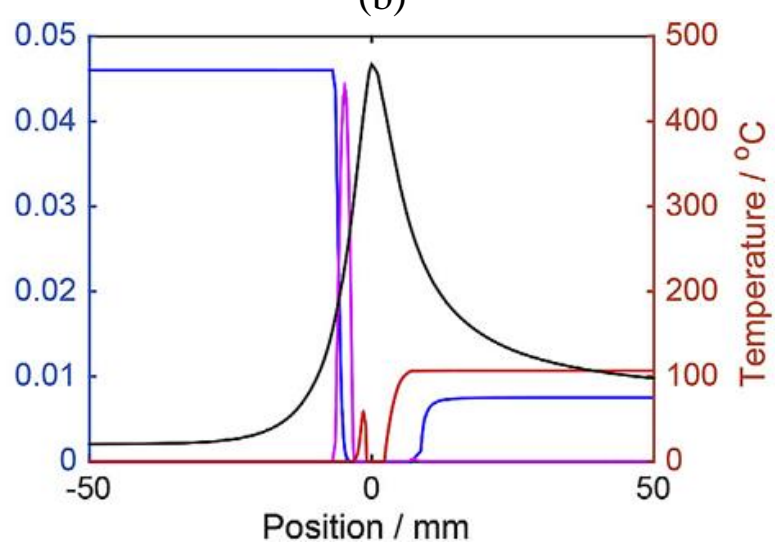

(d)

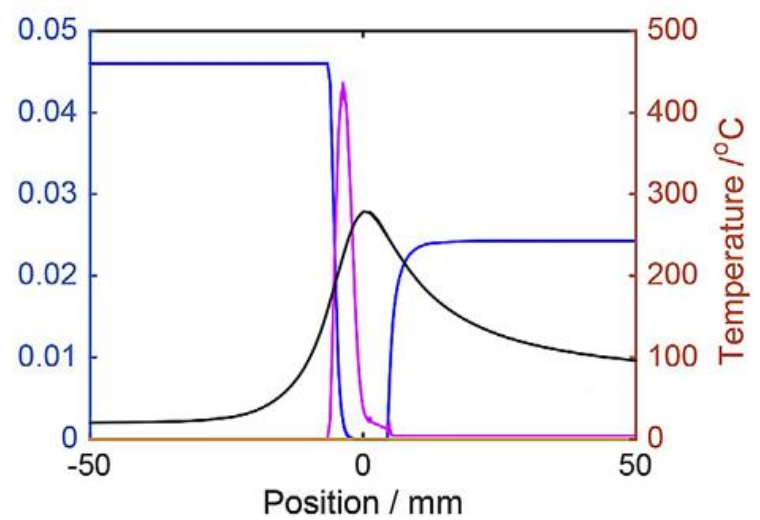

Fig 25: Evolution of precipitate volume fraction (three types, $\eta^{\prime}, \eta$ and GB- $\eta$ ) in 7449-TAF depending from the distance ahead (negative value) or behind (positive value) the tool centre. The positions corresponding to PSD simulations in metal are respectively selected as (a) weld centreline (0 mm) and at (b) $2 \mathrm{~mm}$, (c) $4 \mathrm{~mm}$ and (d) $7 \mathrm{~mm}$ (edge of HAZ) from centreline. (Dos Santos et al., 2018)

\section{III.3. Grain evolution modelling}

In FSW processing, aluminium alloys undergo hot forming in which dynamic recrystallization (DRX) systematically occurs in SZ. The weld microstructure and mechanical properties are deeply related to the DRX phenomena. As demonstrated by McNelley et al. (2008), the main effect of DRX phenomenon is the creation of an equiaxed refined and homogenous microstructure. Several authors tried to forecast the grain size evolution during FSW, but it 
remains many controversies about mechanisms occurring during the process as mentioned by Huang and Logé (2016). According to Humphreys and Hatherly (2004), the Discontinuous Dynamic Recrystallization (DDRX) is mainly observed in low and medium Stacking Fault Energy (SFE) materials as for instance 304 stainless steel, where nucleation of annealed grains occurs during the strain. However, Doherty et al. (1997) mentioned that aluminium alloys are considered as a high SFE alloy. In this condition, two other kinds of DRX are commonly accepted corresponding to Continuous Dynamic Recrystallization (CDRX) and Geometric Dynamic recrystallization (GDRX).

The CDRX is characterized by a grain fragmentation occurring by the formation of new grain boundaries as explained by Gourdet and Montheillet (2003). Huang and Logé (2016) consider that new grains with High Angle Grain Boundaries (HAGBs) are formed during the deformation by the progressive rotation of subgrains with Low Angle Grain Boundaries (LAGBs). This mechanism generates reduced, relatively homogeneous and equiaxed grains that could be much smaller than the initial one. The GDRX is based on the idea that the deformed grain becomes elongated after large deformations until the generated serrations become pinched off and new equiaxed grains thus appear. The grain size refinement is the consequence of the elongation and thickness decrease of grains. Only few numerical models are used for the CDRX and even less for the GDRX. Consequently the review of the different models dedicated to grain size evolution in the SZ is often limited to these both approaches. The GDRX mechanisms are generally well known. However its modelling is usually based on simple geometrical hypotheses considering cubic or spherical grain shapes for instance as proposed by Gholinia et al. (2002) regarding an $\mathrm{Al}-3 \mathrm{Mg}-0.2 \mathrm{Cr}-$ 0.2Fe alloy. Gourdet et al. (1996) applied similarly hypotheses based on MacQueen GDRX theory. More complex shapes are assumed by De Pari and Misiolek (2008) who used a model based on a truncated octahedron providing also significant results.

The prediction of the thermomechanical history can be achieved by Finite Element or analytical models and coupled to the microstructural evolution. This later can be developed with fully analytical models or more original methods such as the cellular automaton (CA) or the Monte Carlo (Potts models) approaches as detailed hereafter. With regard to the modelling of grain size evolution, one can distinguish between (i) material models based on physical properties and more physical evolution laws based on DDRX, GDRX or CDRX models, (ii) empirical methods, mainly used in Cellular Automaton - Finite Element (CAFE) models which are easy to implement but require important and tedious calibration steps (one condition requires one test), (iii) Monte Carlo methods where final observations have to be considered as one possible evolution (stochastic simulation). Consequently, different recrystallization models and their associated results are reported hereafter. These models are also summarized in table 3 with their main features. We first focus on DDRX models and their applications in modelling of microstructure evolutions during stirring processes applied on aluminium alloys. A GDRX model is then presented. CDRX models are afterward detailed considering recent applications. Monte-Carlo methodology and application are finally 
developed in the last part. These latter models are not clearly categorized by their authors in one of the previous approaches and are consequently separately detailed. 
Table 3: Models dedicated to the simulation of grain structure evolutions in FSW processes as applied on aluminium alloys:

\begin{tabular}{|c|c|c|c|}
\hline $\begin{array}{l}\text { Models } \\
\text { and References }\end{array}$ & Alloys & Approach & Remarks \\
\hline $\begin{array}{l}\text { DDRX model } \\
\text { Derby-Ashby approach } \\
\text { (Derby and Ashby 1987, } \\
\text { Hines and Vecchio 1997, } \\
\text { Hofmann and Vecchio 2007) }\end{array}$ & AA6061 & $\begin{array}{l}\text { Linear-time evolution for grain growth } \\
\text { evolution, dependence with grain } \\
\text { boundary mobility leading to analytical } \\
\text { expression of grain size }\end{array}$ & $\begin{array}{l}\text { Grain size modelling from experimental } \\
\text { measurement of thermal evolution, Final } \\
\text { grain diameter in stirred material based on } \\
\text { boundary migration model }\end{array}$ \\
\hline $\begin{array}{l}\text { DDRX model, } \\
\text { Zener-Hollomon approach } \\
\text { (Wan et al. 2017) }\end{array}$ & AA6082-T6 & $\begin{array}{l}\text { Grain diameter linked to the Z-H } \\
\text { parameter with a power law relation } \\
\text { also influenced by initial grain size. Z-H } \\
\text { parameter influenced by temperature } \\
\text { and strain rate. }\end{array}$ & $\begin{array}{l}\text { Use of randomly-distributed particle tracers to } \\
\text { estimate normal-distribution of final grain } \\
\text { size. Effect of process parameter investigated } \\
\text { on this distribution, Fully analytical } \\
\text { modelling, time-efficiency approach }\end{array}$ \\
\hline $\begin{array}{l}\text { DDRX model, } \\
\text { Avrami approach } \\
\text { (Khalkhali et al. 2015, } \\
\text { Shojaeefard et al. 2014) }\end{array}$ & $\begin{array}{l}\text { AA7050 } \\
\text { AA1100 }\end{array}$ & $\begin{array}{l}\text { The Avrami equation describes the } \\
\text { relationship between the recrystallized } \\
\text { fraction and the effective strain. }\end{array}$ & $\begin{array}{l}\text { Based on grain nucleation or growth } \\
\text { mechanism depending from a threshold value } \\
\text { (dislocation density - Shojaeefard et al. (2014) } \\
\text { and strain - Khalkhali et al. (2015)) leading to } \\
\text { DDRX. On the contrary, grain growth } \\
\text { mechanism takes place in aluminium alloy. }\end{array}$ \\
\hline $\begin{array}{l}\text { GDRX model } \\
\text { (Robson and Campbell 2010, } \\
\text { Humphreys and Hatherly } \\
\text { 2004, Prangnell and Heason } \\
\text { 2005) }\end{array}$ & AA2524 & $\begin{array}{l}\text { High-angle grain boundary depending } \\
\text { from the shear strain and subgrain size } \\
\text { depending from the Z-H parameter with } \\
\text { an inverse-logarithmic relation. Z-H } \\
\text { parameter influenced by temperature } \\
\text { and strain rate. }\end{array}$ & $\begin{array}{l}\text { Grain size after recrystallization. Investigation } \\
\text { of the dispersoid particles and cooling rate } \\
\text { influences. In the Mac Queen model, the } \\
\text { strain is assumed to 'pinch' the initial grain. }\end{array}$ \\
\hline $\begin{array}{l}\text { CDRX model } \\
\text { Empirical modelling } \\
\text { (CAFE method) } \\
\text { (Saluja et al. 2012, } \\
\text { Valvi et al. 2016) }\end{array}$ & $\begin{array}{l}\text { Al6061T6 / } \\
\text { Al6061T6 and } \\
\text { Al6061T6/A150 } \\
860 \\
\text { (similar / }\end{array}$ & $\begin{array}{l}\text { CAFE model with analytically } \\
\text { estimated strain rate field imposed onto } \\
\text { material, Transition rules between cells } \\
\text { to predict grain size, Empirical model } \\
\text { requiring calibration procedure }\end{array}$ & $\begin{array}{l}\text { Dislocation density, tensile behaviour and } \\
\text { micro-features prediction, accuracy of stress- } \\
\text { strain evolution shown, Development and } \\
\text { Influence of weld defects, Development on } \\
\text { Abaqus6.8 }\end{array}$ \\
\hline
\end{tabular}




\section{dissimilar}

grades)

\section{CDRX model}

Physical modelling

(Gourdet-Montheillet

approach)

(Gourdet and Montheillet

2003, Jacquin 2009)
Modelling of dislocation density

evolution inside grains, Calibrated

AA2024

parameters, Particle tracking during

FSW process to follow grain size

evolution
Grain size estimation in various welding

conditions and comparisons with experiments.

Dislocations generated during deformation

rearrange themselves to produce new grain boundaries (G-M model)

\section{Monte-Carlo}

(Grujicic et al. 2015,

Zhang et al. 2016)
AA5083-H321,

AA6082-T6
Random process and transition rules, nucleation phenomena introduced to model recrystallization process, initial anisotropic grain structure
Grain evolution in TMAZ, HAZ and SZ,

depth-dependence of grain structure

evolution, experimental comparison,

investigation of process parameters effects 


\section{III.3.a. DDRX modelling}

\section{- Derby and Ashby recrystallization approach}

The literature dedicated to the modelling of microstructure evolution in FSW provides few source clearly based on DDRX approach including nucleation of new grains in aluminium alloys during stirring processes. However we have to mention the activities developed by Hofmann and Vecchio (2007) in order to apply the Derby-Ashby (1987) model to cooling curves obtained from thermocouples data when measuring temperature in stirred aluminium alloys. This model is clearly associated to DDRX phenomena and corresponds to one of the single activities to our knowledge reported in the literature to model DDRX phenomena during stirring of aluminium alloys.

\section{a. Presentation}

Two processes were investigated by Hofmann and Vecchio corresponding to Friction Stir Processing (FSP) and Submerged Friction Stir Processing (SFSP) developed on AA6061 aluminium grade. These processes aim at developing a severe plastic deformation on material to produce bulk samples with fine-grained microstructure. FSP and SFSP processes have to be considered as different from FSW processes which are investigated in the present article. However, FSP and FSW processes also lead to the development of large recrystallization mechanisms when material undergone high stirring mechanism. In both case, recrystallization is induced by temperature and strain evolutions onto the material leading to new microstructure. Consequently the present section will focus on the application of DerbyAshby model to follow grain structure evolution in FSP also considering the possibility to apply similar approach on FSW processes in future development.

\section{b. Methodology}

In Derby-Ashby model, the time required to growth grain up to a diameter $S$ is a linear function of the migration rate, $g$, associated to grain boundary:

$t=\frac{S}{2 g}$

where the grain migration rate is assumed as the product of the driving force and grain boundary mobility. The grain boundary mobility, $M$, is itself dependent on the grain boundary diffusivity, $D$, the boundary layer thickness, $\delta$, and Burgers vector of dislocations, $b$, also including temperature, $T$, dependence. The boundary diffusivity evolves as an Arrhenius law with an activation energy $Q$. The driving force, $F$, is the store work energy in subgrain walls, 
depending from the misorientation angle between grains, $\theta$, the shear modulus, $\mu$, and the Burgers vector. The following expressions are thereafter obtained:

$$
\left\{\begin{array}{c}
M=\frac{b \delta}{k T} D_{0} e^{-\frac{Q}{R T}} \\
F=\frac{3 \mu b \theta}{L}
\end{array}\right.
$$

Hofmann and Vecchio (2007) rearrange the grain size evolution expression provided by expression (25-26) and older expression previously developed by Hines and Vecchio (1997) to provide an analytical time-dependent expression considering temperature evolution in friction stir processing:

$S=\frac{t(T) 6 \delta b^{2} \mu \theta}{L k T} D_{0} e^{-\frac{Q}{R T}}$

\section{c. Results}

Hofmann and Vecchio (2007) rightly point the difficulty to use such model in grain size estimation for large cooling rate as encountered at micro-scale in shear band cooling. However, this model is appropriate and suitable to describe grain growth in FSP, SFSP and FSW processes when cooling evolution is of the order of $\sim 300{ }^{\circ} \mathrm{C}$ in $20 \mathrm{~s}$ preventing underestimation of microstructure size. Model application is done on both FSP and SFSP process on AA6061-T6 thick plates with initial grain size of $50 \mu \mathrm{m}$. This model (Eq. 27) was employed to determine final grain size based on experimental measurements of thermal history obtained with thermocouples placed in the stirred material. Evolutions are reported on Fig. (26) for four experiments also considering the effect of plunging stage on material. As shown by the author, good comparison (Fig. 26 b-c) is obtained in final grain size prediction for experiments conducted with plunging stage (Fig. 26 a) leading to large temperature and low cooling rate. Grain sizes are respectively of $5.6 \mu \mathrm{m}$ and $4.1 \mu \mathrm{m}$ in FSP and SFSP processes corresponding to values close to experimental observations (Fig. 26 c). Smaller grain sizes are achieved when plunging stage is not considered. Derby-Ashby model predicts grain size of $134 \mathrm{~nm}$ and $1 \mathrm{~nm}$ which are clearly smaller than measurements and demonstrates the need to include plunging stage in modelling approach. The authors also demonstrate the interest to develop the alternative SFSP processes to obtain smaller grain size with same stirring conditions. In addition, larger cooling rates and lower temperatures are obtained when water is used to absorb residual frictional heat. The grain development in aluminium alloys is limited leading to better mechanical properties associated to a finer grain microstructure. 
(a)

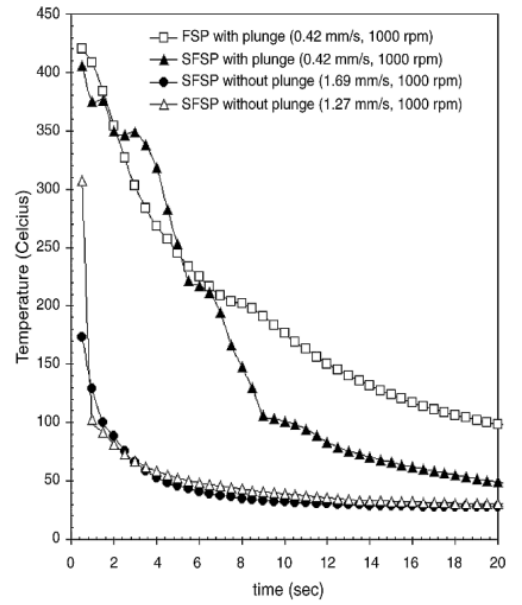

(b)

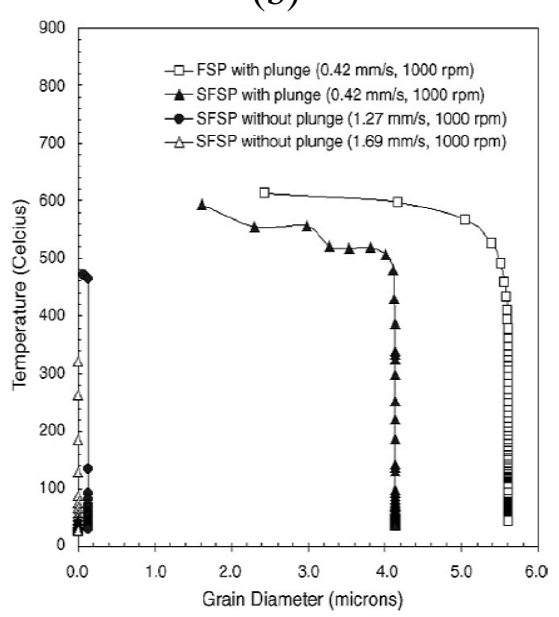

(c)

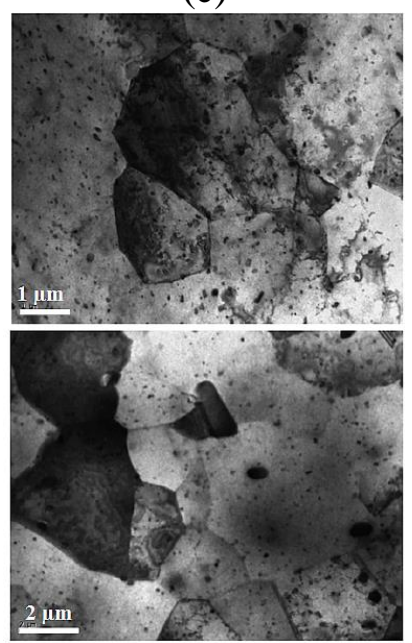

Fig. 26: (a) Heat flow curves from bottom part of the sample obtained in FSP and SFSP processes including or not a plunging stage. (b) Grain structure evolution associated to same cooling curves and based on the analytical model of Derby and Ashby (1987). (c) Experimental observation reported for plunging test (Hofmann and Vecchio, 2007).

\section{- Zener-Hollomon approach}

\section{a. Presentation}

Wan et al. (2017) propose to model grain size evolution during FSW using a Zener-Hollomon (Z-H) parameter. This approach is related to the nucleation of new grains on current grain boundaries and also corresponds to physical processes occurring in DDRX approaches.

\section{b. Methodology}

Wan et al. (2017) model the grain size evolution according to the initial grain size and the strain rate encountered by the grain. A Zener-Hollomon power law connects the final grain size to the strain rate magnitude. Consequently, the Zener-Hollomon parameter determines the final size of grains. The following relation is then provided by Wan in order to estimate final microstructure size in a AA6082-T6 FSW sample:

$\ln \left(\frac{d}{D}\right)=1.81-0.23 \ln Z$

where $D$ is the initial grain size, equal to $80 \mu \mathrm{m}$, and $Z$ is the Zener-Hollomon parameter. This latter value is then estimated as:

$Z=\dot{\bar{\varepsilon}} \mathrm{e}^{\frac{Q}{R T}}$

where $\dot{\bar{\varepsilon}}$ is the equivalent strain rate, $Q(180 \mathrm{~kJ} / \mathrm{mol})$ is the material activation energy, $R$ is the gas constant, and $T$ is the absolute temperature. $\dot{\bar{\varepsilon}}$ is extracted from a simulate material flow 
using numerical point tracking technique based on Finite Element method and remeshing approach. A set of randomly-distributed particle tracer is used to estimate normal-distribution of final grain size. Effects of process parameter are investigated on this distribution.

\section{c. Results}

Fig. 27 shows the distribution of grain sizes and associated probability functions considering various welding conditions obtained by Wan et al. (2017). The predicted grain sizes vary from 9.32 to $9.62 \mu \mathrm{m}$ near the top surface and from 8.29 to $8.84 \mu \mathrm{m}$ close to the bottom surface. The average grain size on both top and bottom surfaces increases when increasing the rotational speed. Indeed, the mean value of grain size evolves from $9.11 \mu \mathrm{m}$ at $715 \mathrm{rpm}$ rotating speed to $27.5 \mu \mathrm{m}$ at $1500 \mathrm{rpm}$. The authors explain that for higher rotational speeds, cooling takes a more important place in the thermal cycle, and particles with longer cooling duration undergo longer recovery and grain growth after recrystallization.

(a)

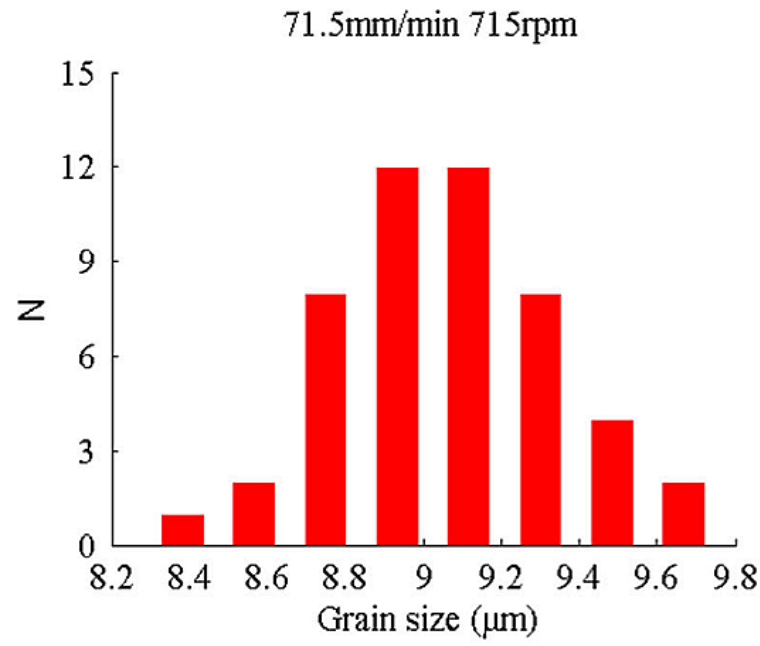

(c)

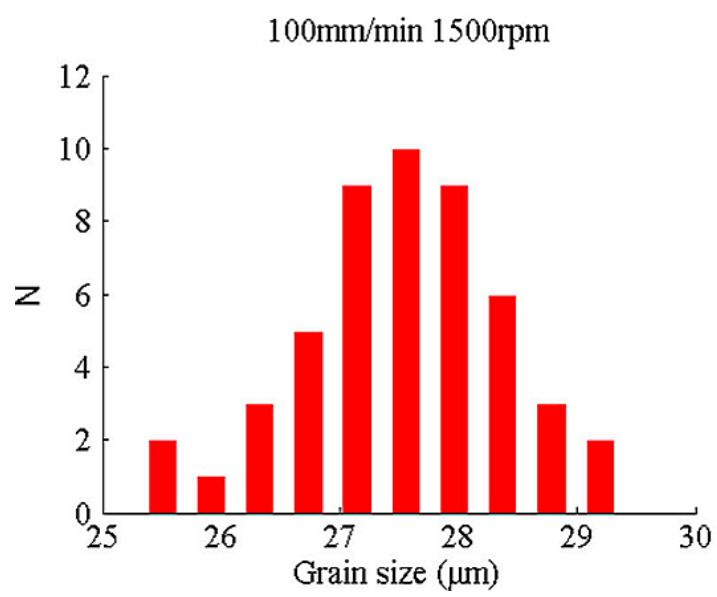

(b)

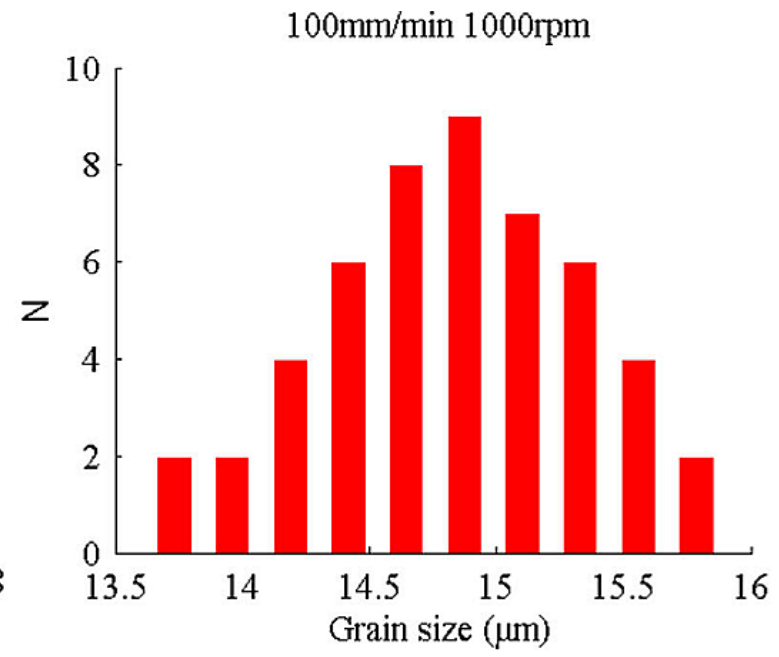

(d)

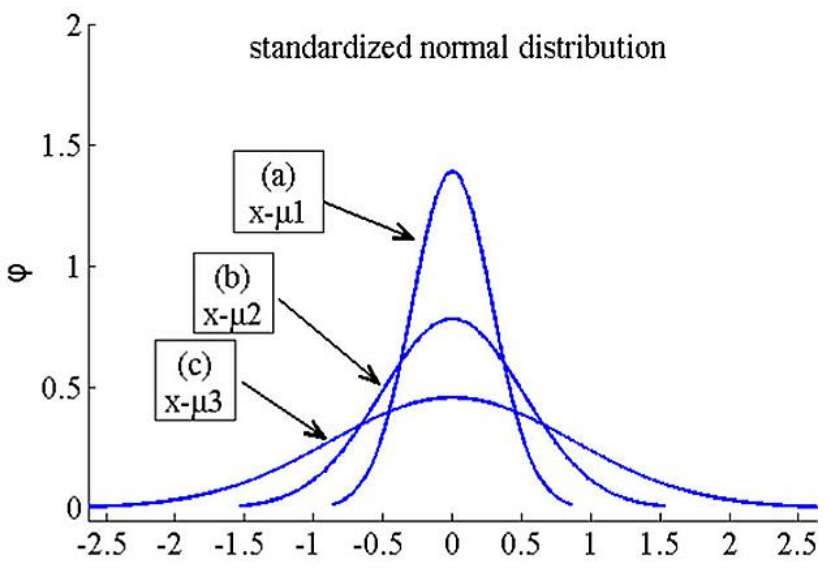

Fig. 27: (a-c) Statistical grain sizes and (d) probability density functions in different welding conditions after selection of fifty material particles inside the stir zone in each case. (Wan et al., 2017) 


\section{- Avrami model approach}

\section{a. Presentation}

Shojaeefard et al. (2014) used an Avrami model provided by DEFORM-3D ${ }^{\mathrm{TM}}$ (DEFORM, 2019) software coupled with numerical strain, strain rate, stress and temperature field to predict microstructural evolution during FSW process. A CA method is coupled with a modified Laasraoui Jonas model to simulate recrystallization mechanism before developing experimental comparison. The model and its application are focused on aluminium grades AA1100. Khalkhali and Saranjam (2015) proposed similar methodology however focused on aluminium grades AA7050 of industrial interest for automotive industries. The average grain size and associated recrystallized grain fraction are predicted in the nugget domain considering the recrystallization mechanism model. The microstructure evolution model proposed by DEFORM-3 $\mathrm{D}^{\mathrm{TM}}$ is based on both nucleation rate and grain growth kinetics. Considering the hypothesis and model associated to grain growth mechanism, such approach can be categorized as a DDRX approach.

\section{b. Methodology}

Khalkhali et Saranjam (2015) propose to initiate the dynamic recrystallization with the plastic strain parameter following the approach of Yi et al. (2008). The plastic strain value cannot exceed a critical strain $\varepsilon_{c}\left(\varepsilon_{c}=0.8 \varepsilon_{p}\right)$. The onset of dynamic recrystallization occurs consequently for a strain magnitude equal to $80 \%$ of this $\varepsilon_{p}$ value expressed as:

$\varepsilon_{p}=a_{1} \dot{\varepsilon}^{m_{1}} e^{\frac{Q_{1}}{R T}}$

where the activation energy, $Q_{1}$, is equal to $1.318 \cdot 10^{4} \mathrm{~J} \cdot \mathrm{mol}^{-1} . a_{1}$ is a given coefficient and $m_{1}$ is a grain-strain rate exponent. The experimental values of $a_{1}$ and $m_{1}$ are respectively $4.107 \cdot 10^{-3}$ and 0.06 . All these data are reported in the literature by Yi et al. (2008) and obtained from experimental analysis previously developed when forging aluminium alloy 7050. Same reference also provides the expression detailed hereafter. $\dot{\varepsilon}$ represents the strain rate and is given as:

$\dot{\varepsilon}=A\left[\sinh \left(\alpha \sigma^{p}\right)\right]^{n} e^{-\frac{Q}{R T}}$

where $A, \alpha$ and $n$ are constants values considered as temperature independent. $\sigma^{p}$ is the peak stress and $Q$ is the activation energy associated to hot deformation, $R$ represents the universal gas constant. $A, \alpha, n$ and $Q$ values are respectively equal to $5.83 \cdot 10^{18} \mathrm{~s}^{-1}, 0.01239 \mathrm{~Pa}^{-1}, 7.598$ and $2.6406 \cdot 10^{5} \mathrm{~J} \cdot \mathrm{mol}^{-1}$. The dynamically recrystallized fraction $X_{D R X}$ is computed by the Avrami equation according to the effective strain with relation: 
$X_{D R X}=1-e^{-\beta_{d}\left(\frac{\varepsilon-\varepsilon_{c}}{\varepsilon_{0.5}}\right)^{2}}$

where $\beta_{d}$ is a material data experimental coefficient and $\varepsilon_{0.5}$ represents the strain associated to a volume fraction of recrystallized grains equal to $50 \%$. This latter is expressed as:

$\varepsilon_{0.5}=a_{5} d_{0}^{h_{5}} \dot{\varepsilon}^{m_{5}} e^{\frac{Q_{5}}{R T}}$

The value $d_{0}$ represents the initial grain size before recrystallization obtained from experimental measurements. Then, the authors express the size of recrystallized grain with a relation depending from the strain, strain rate and temperature, also considering the initial grain size:

$d_{D R X}=a_{8} \dot{\varepsilon}^{m_{8}} e^{\frac{Q_{8}}{R T}}$

The average grain size can be computed by a relation corresponding to a balance between initial grain, $d_{0}$, and recrystallized grain sizes, $d_{D R X}$ :

$d=d_{0}\left(1-X_{D R X}\right)+d_{D R X} X_{D R X}$

All the dynamic recrystallization coefficients concerning the AA7050 are reported in table 4 and provide from the careful experimental analysis developed by Yi et al. (2008).

Table 4: Dynamic recrystallization coefficients as proposed by Khalkhali and Saranjam (2015) and Yi et al. (2008)

\begin{tabular}{c|cccc}
\hline Name & $\beta_{d}[-]$ & $Q_{5}\left[\mathrm{~J} \cdot \mathrm{mol}^{-1}\right]$ & $Q_{8}\left[\mathrm{~J} \cdot \mathrm{mol}^{-1}\right]$ & $a_{5}[-]$ \\
\hline Material data & 0.693 & $5.335 \cdot 10^{-4}$ & -19002.72 & $1.214 \cdot 10^{-5}$ \\
\hline \hline Name & $a_{8}[\mu \mathrm{m}]$ & $h_{5}[-]$ & $m_{5}[-]$ & $m_{8}[-]$ \\
\hline Material data & 78.6022 & 0.13 & 0.04 & -0.03722 \\
\hline \hline
\end{tabular}

Shojaeefard et al. (2014) also used the DEFORM-3D ${ }^{\mathrm{TM}}$ software to compute the average recrystallized grain size. They assume that the dislocation density is the main phenomenon producing nucleation phenomenon and grain size evolution. If the dislocation density, $\rho_{i}$, reaches a threshold value, $\rho_{c}$, then the dynamic recrystallization occurs. On the contrary, if the $\rho_{i}$ value is beneath $\rho_{c}$ then the grain growth mainly takes place in the material. The dislocation density is calculated with Laasraoui-Jonas model as presented in equation (46) detailed hereafter in part III.3.c. The flow chart of calculation process is given in Fig. 28. 


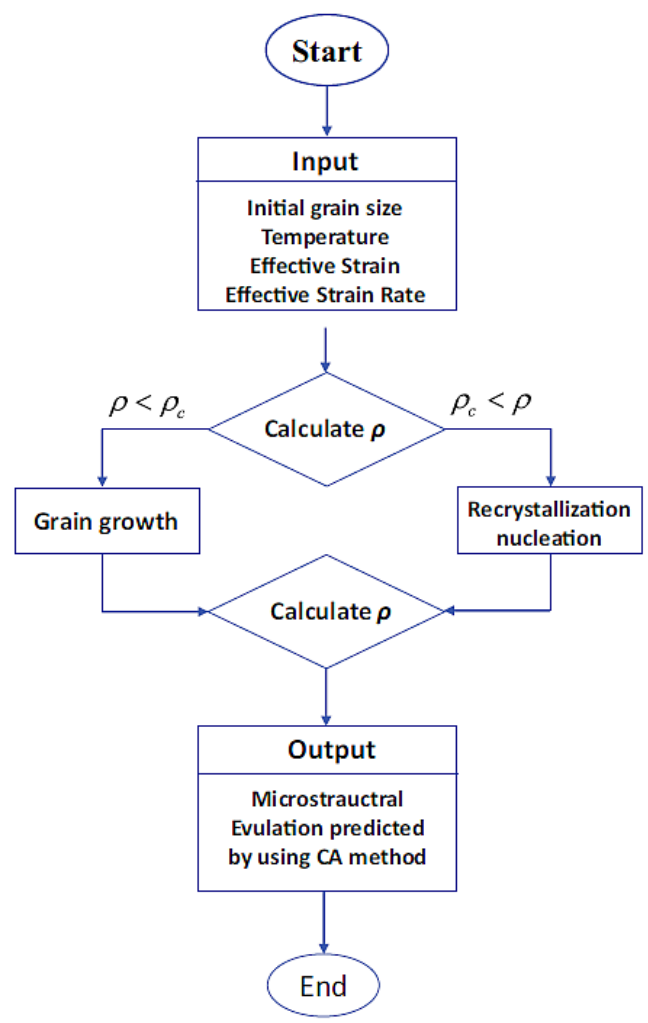

Fig. 28: Flow chart of calculation process for microstructural evolution (Shojaeefard et al., 2014)

c. Results

Khalkhali and Saranjam (2015) model is active when strain and temperature reach critical values at the same time. The volume fraction of dynamic recrystallized grain increases when the strain and temperature increase as a consequence of chosen process parameters. Fig. 29 a) shows the computed recrystallized grain size for different tool rotational velocity. The authors demonstrate a good agreement of their simulation with experimental results in the stir zone where they observe refined equiaxed grain with a size of around $2.5 \mu \mathrm{m}$. The authors show also that when the tool rotation speed decreases, the final recrystallized grain size reduces. In addition, the set of results obtained by Shojaeefard et al. (2014) on AA1100 aluminium grades is presented in Fig. 29 b). As detailed in this comparison, the authors demonstrate that a good agreement between simulations and experimental measurements is achieved in the stir zone. However, we have to point out that the measurement procedure was not clearly detailed by authors. 
(b)

(a)

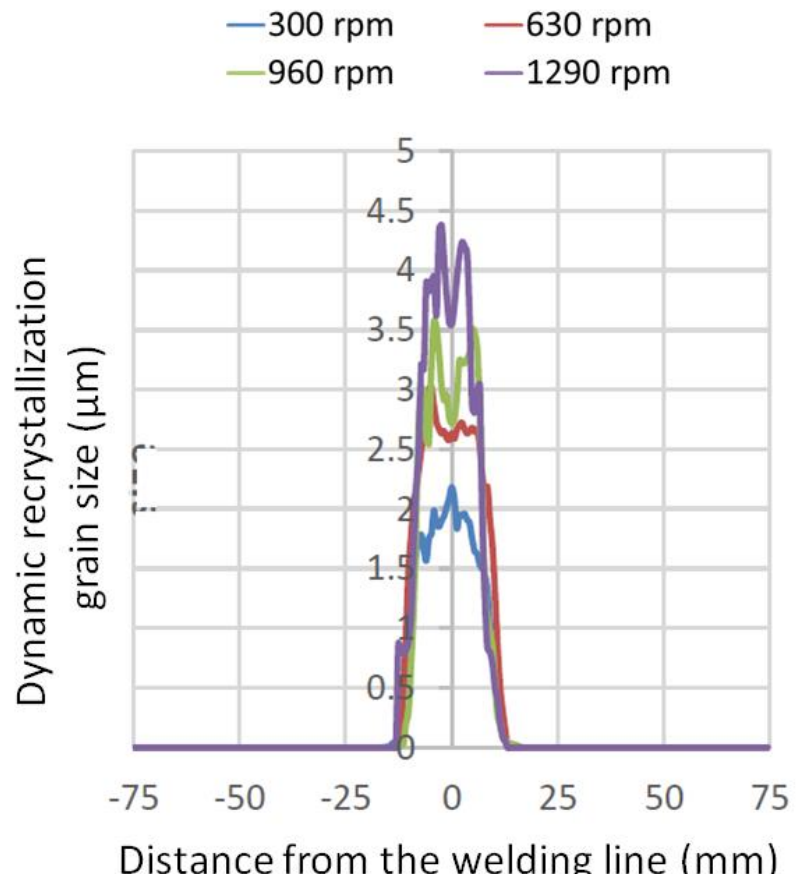

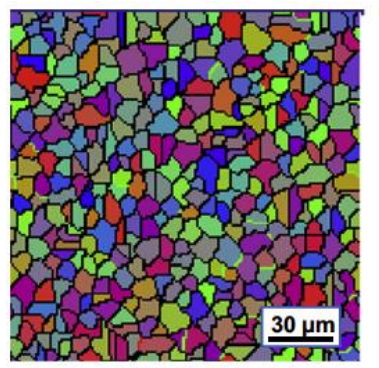

GS=5.7 $\mu \mathrm{m}$

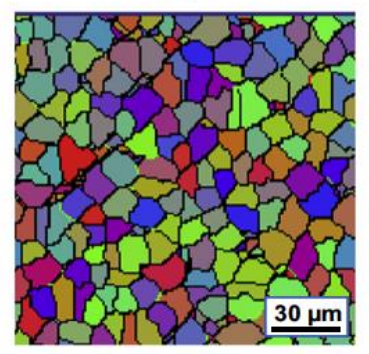

$\mathrm{GS}=8.7 \mu \mathrm{m}$

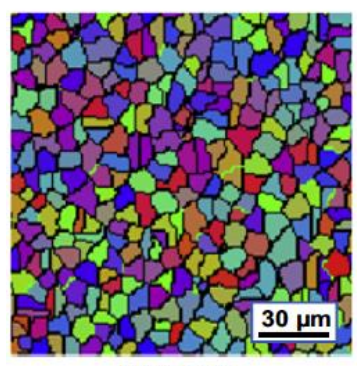

$\mathrm{GS}=6.3 \mathrm{um}$

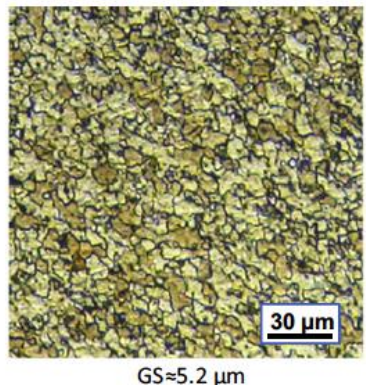

$\mathrm{GS} \approx 5.2 \mu \mathrm{m}$

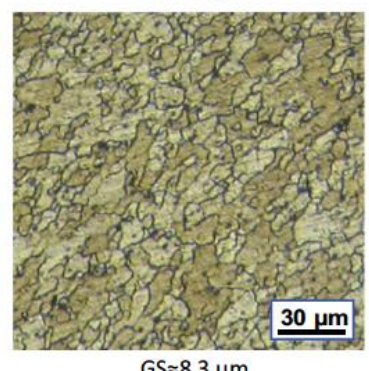

$\mathrm{GS} \approx 8.3 \mu \mathrm{m}$

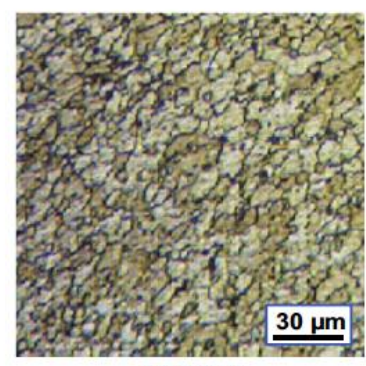

$\mathrm{GS} \approx 6.1 \mathrm{um}$

Fig. 29: (a) AA7050 - Dynamically recrystallized grain size for tool rotational velocity respectively equal to 300, 630, 960 and $1290 \mathrm{rpm}$ (Khalkhali and Saranjam, 2015). (b) AA1100 - Simulated and experimental microstructure of stir zone for rotational speed, traverse speed and shoulder diameters respectively equal to (first line) $900 \mathrm{rpm}, 16 \mathrm{~mm}$ and $120 \mathrm{~mm} \cdot \mathrm{min}^{-1}$, (second line) $1120 \mathrm{rpm}, 14 \mathrm{~mm}$ and $120 \mathrm{~mm} \cdot \mathrm{min}^{-1}$, (third line) $900 \mathrm{rpm}, 14$ $\mathrm{mm}$ and $80 \mathrm{~mm} \cdot \mathrm{min}^{-1}$ (Shojaeefard et al., 2014).

\section{III.3.b. GDRX modelling}

\section{a. Presentation}

Robson and Campbell (2010) propose a recrystallization and grain growth model for microstructure evolution in the stir zone. Their approach is based on the GDRX model initially proposed by Prangnell and Heason (2005), and the development of a process model able to predict the main thermomechanical fields within the nugget zone. All the tests and computation have been calibrated for AA2524 aluminium alloys. Furthermore, the results 
have been interestingly extended to the investigation of dispersoid particles influence and cooling rate effect. Grain coarsening after stirring is also considered.

\section{b. Methodology}

The usual assumption for GDRX modelling is used. Robson and Campbell assume that the GDRX phenomenon starts when the stirring of the material begins. The grain boundary diameter $D_{H A G B}$ (HAGB: high-angle grain boundary) is linked to the shear strain as:

$D_{H A G B}=\frac{D_{0}}{\left(1+\varepsilon_{\text {shear }}^{2}\right)^{1 / 2}}$

where $D_{0}$ and $\varepsilon_{\text {shear }}$ are respectively the original boundary width and the simple shear strain. The subgrain size is provided by the Zener-Hollomon $Z$ parameter as:

$D_{S G}=\frac{1}{a+b \ln Z}$

where $a$ and $b$ correspond to experimentally derived constants. The $Z$ parameter is then provided as:

$Z=\dot{\varepsilon} e^{\frac{Q}{R T}}$

where $\dot{\varepsilon}$ is the strain rate, $Q$ is the activation energy, $R$ is the gas constant. The subgrain size depends from the temperature and strain rate. Consequently, the grain boundary width is reduced according to the following criteria:

- $D_{H A G B}<D_{S G} \quad$ un-recrystallized

- $D_{H A G B}=D_{S G} \quad$ critical condition for recrystallization

- $D_{H A G B}>D_{S G} \quad$ recrystallized

Those criteria determine the onset of recrystallization assuming that this phenomenon instantaneously occurs when critical point is reached. The "pinching off" is supposed to produce grains with same diameters than $D_{S G}$. After the stirring phase, the material located behind the tool stays still at high temperature. Consequently, Robson and Campbell assume that the fine recrystallized grains grow post-dynamically. To take into account this grain growth effect after deformation, the authors use the Humphreys and Hatherly (2004) model consisting in the evaluation of the grain radius $R$ according to the time as provided by the following relation:

$\frac{d R}{d t}=M\left(\frac{\alpha \gamma_{b}}{R}-\frac{3 V_{f} \gamma_{b}}{2 r}\right)$ 
where $M$ is the mobility of high angle grain boundary, $\alpha$ corresponds to a geometric constant and $\gamma_{b}$ is the high angle grain boundary energy. $V_{f}$ is the volume fraction of pinning particles (second phase particles) of radius $r$ limiting grain growth. Arora et al. (2009) model provides calculation method for the thermal and strain rate data input of the present model when applied on AA2524 alloy (Fig. 30). All the calibration data have been collected in the literature for AA2524 or other similar aluminium alloys. The authors have also taken into account the pinning pressure effect induced by dispersoids particles on grain boundaries.

(a)

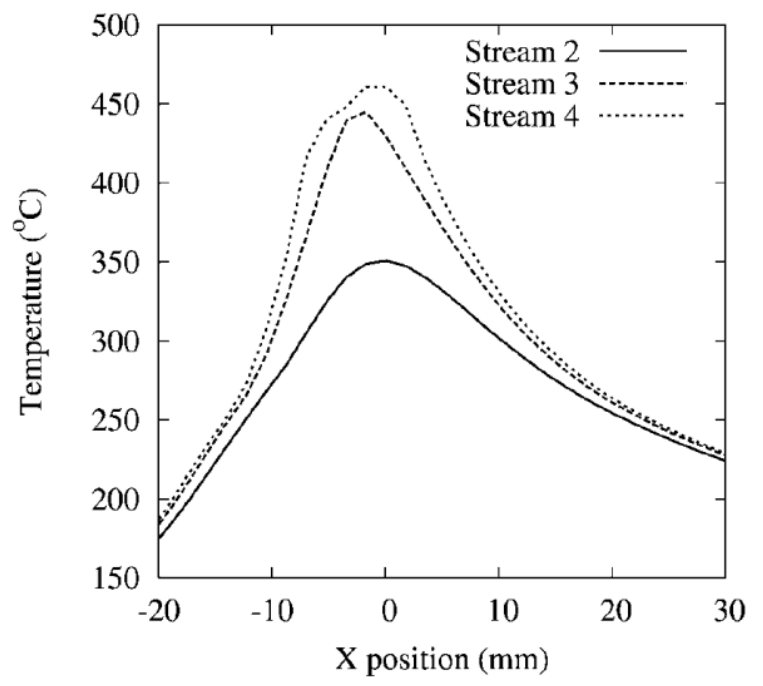

(b)

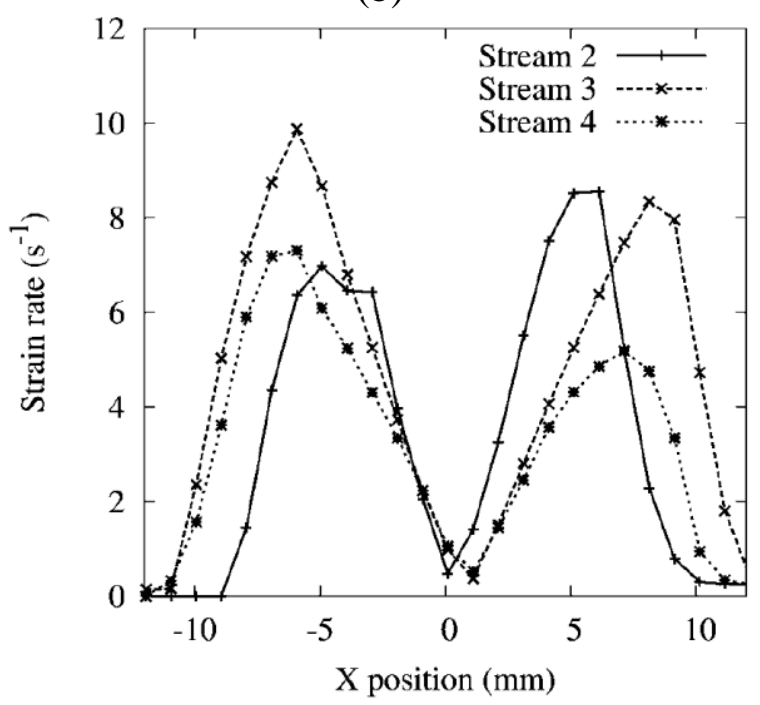

Fig. 30: (a) Temperature evolutions for three streamlines passing retreating side of the tool (streamline numbers are provided by Arora et al. (2009)). (b) Simulation of strain rate evolutions on the same streamlines (Arora et al., 2019) (pin, centre is at $x=0$ and tool translates from positive to negative $x$ ) (Robson and Campbell, 2010)

\section{c. Results}

The main computation results are shown in Fig. 31. As expected, grain size reduces rapidly to roughly $0.9 \mu \mathrm{m}$ in the neighbourhood of the stir zone. Then, the grain size grows up following an exponential curve corresponding to the post dynamic grain growth. Fig. 31 a) shows that the kinetic modelling of the post-dynamic grain growth is very fast. Grains reach their final size quasi instantaneously behind the pin. The obtained computed results are in good agreement considering experimental measurements. The authors tried also to avoid the introduction of any arbitrary fitting parameters as much as possible. Nevertheless, their model is highly dependent on the material parameters chosen by users as well as the accuracy of the estimated thermomechanical fields. In addition, the authors investigate the influence of dispersoid particles which are stable during FSW process. They show that an overestimation of $50 \%$ in final grain size is observed (Fig. 31 b) when these latter are not considered in the approach. 
(a)

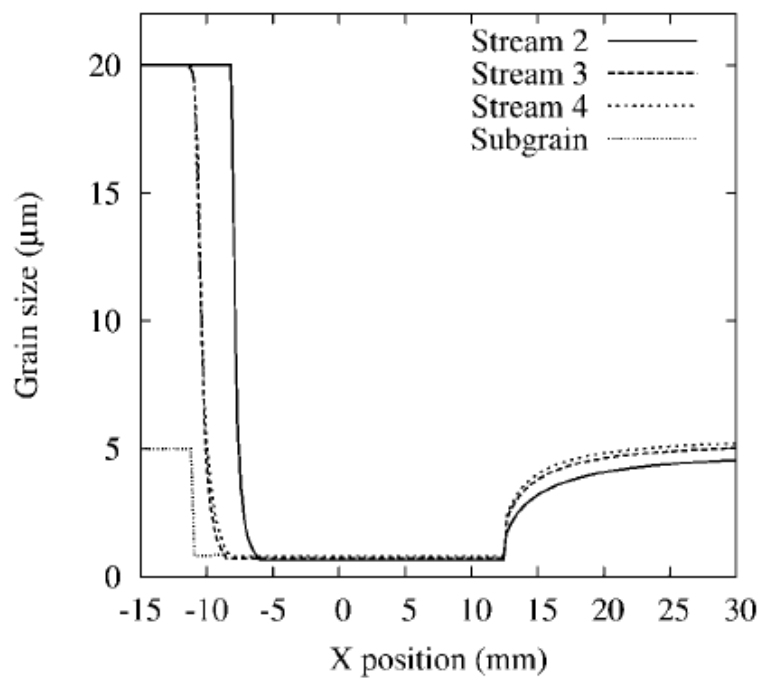

(b)

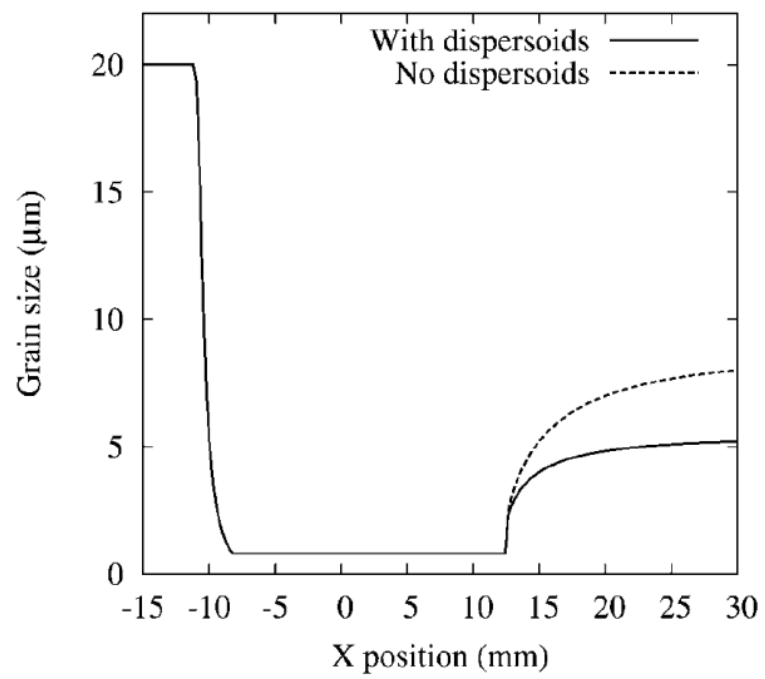

Fig. 31: Predicted evolution of grain size (a) for various streamlines (2-4) also considering subgrain size (streamline 4) and (b) for streamline 4 with and without influence of dispersoid particles (Robson and Campbell, 2010).

\section{III.3.c. CDRX modelling}

\section{- Empirical model}

\section{a. Presentation}

Numerous activities are reported in literature dealing with the modelling of grain structure evolution in materials processing based on Cellular Automaton - Finite Element (CAFE) coupling approaches as reported by Schmitz and Prahl (2016). This method has been widely used in order to model grain evolution regarding the literature devoted to materials science. Carozzani et al. (2012) applied the CAFE model in casting processes to simulate nucleation and grain growth also considering experimental validation. Chen et al. (2016) extend similar methodology to simulate welding processes in a level set approach and model epitaxial grain growth with relevant results. More recently, Pineau et al. (2018) compared boundary orientation obtained after grain growth competition to phase field simulations in a wide range of grain orientation angles. The efficiency of CAFE models to mimic these competition mechanisms was demonstrated when relevant cell size is preliminary chosen. In thermomechanical processes, Das (2010) has firstly proposed a multi-level cellular automata framework able to capture features in steel developed at micro-scale during strain compression. This work responds to the need to model structure development at micro-scale (CA) compared to the macro-scale (FE) solution usually applied to model thermo-mechanical evolution in materials processing. In this direction, the CA approaches represent the way to 
follow grain evolution at small scale when complex phenomena occurs on each individual structure considering that material evolution is known at a larger scale. Das also coupled its own modelling in the commercial software Abaqus ${ }^{\circledR}$ in order to benefit from thermomechanical solution. In more recent works reported in literature, the CAFE model has been successfully applied with a similar approach to the modelling of grain structure evolution during forming of FSW aluminium sheets. Saluja et al. (2012) developed a CAFE model aiming at predicting grain size distribution during FSW process. Thermo-mechanical evolution of welded domains is similarly simulated on FE mesh based on an analytical model. Consequently, strain, strain rate and heat flux are considered through analytical expressions. Heat conservation equation is then solved to compute temperature field evolution in a moving coordinate system. Solutions fields are used later onto the CA grid to compute grain structure evolution also extending the previous approach of Das (2010) in three dimensions. This activity was dedicated to similar welding on Al6061T6 aluminium grades. In a complementary part, the development of welding defects has also been investigated considering void cells and their influence on the forming of welded aluminium sheets. Valvi et al. (2016) apply and extend the initial activity of Saluja et al. (2012) afterwards to investigate welding of dissimilar Al alloys (Al6061T6/A15086O) as well as dislocation density evolution. Similarly, the original microstructure is used to create CA grain instead of using conventional CA rules associated to grain growth as detailed afterwards. The end-use application of such modelling is the prediction of final mechanical properties through the weld region which is achieved with experimental observations.

\section{b. Methodology}

The methodology is based on the capability of CA cell approach to model grain development in accordance with local strain, $\varepsilon$, strain rate, $\dot{\varepsilon}$, and temperature, $T$, evolution, leading to the estimation of current yield stress in material. The CA model is schematized on Fig. 32 showing the input and output data. At the part scale, a 3D FE mesh is developed and applied onto the piece. Linear 8-noded hexahedral elements are used to simulate FSW process.

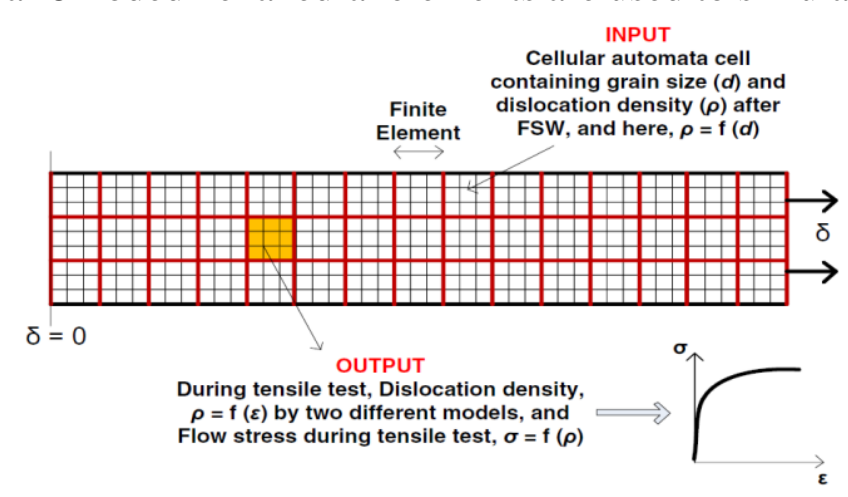

Fig. 32: Flow stress predicted as a function of dislocation density updated with strain during tensile test considering input and output data (Valvi et al., 2016) 
The heat conservation equation is firstly solved considering heat input generated at the tool shoulder interface, pin surface and bottom of the tool pin. The total heat input depends directly from the pin and shoulder geometries. In addition boundary conditions are applied onto the base plate and air contact considering heat extraction induced by external contact of the material. The model has been improved by Valvi et al. (2016) to integrate temperature dependence compared to the initial approach of Saluja et al. (2012). The strain rate distribution in welded zone has not been computed but analytically imposed considering the material flow inside the material as:

$\dot{\varepsilon}=\frac{1}{2}\left(\nabla u+\nabla^{T} u\right) \quad$ where $\quad u=\delta_{(s)} u_{(s)}+\delta_{(p)} u_{(p)}$

where the components of the velocity field are:

$\left\{\begin{array}{cl}u_{(s) r}=\alpha[-v \sin \theta] & u_{(s) \theta}=\alpha[\omega r-v \cos \theta] \\ u_{(p) r}=\beta[-v \sin \theta] & u_{(p) \theta}=\beta\left[\omega r_{p}-v \cos \theta\right]\end{array}\right.$

The parameters values depend from friction conditions. The overall velocity field is consequently written as a summation of weight velocity functions imposed respectively by shoulder $\left(\delta_{(s)}=0.3\right)$ and pin $\left(\delta_{(p)}=0.7\right)$. This model follows the approach originally proposed by Darras and Khraisheh (2008). The CA approach is initially based on an original microstructure. This latter is scanned and image processing algorithms are used to create CA objects over the whole welded domain. The CA grid is linked onto the FE element with an average number of 25 square cells in each element given with a Gaussian distribution. The transition rules are then applied in the prediction of grain size evolution during FSW process depending from the local value of strain, strain field and temperature based on a power law relation as proposed by Lenard (1999):

$D_{C D R X}=C \varepsilon^{k} \dot{\varepsilon}^{j} D_{i}^{h} \exp \left(-\frac{Q}{R T}\right)$

where $D_{C D R X}$ is the average grain size, $\varepsilon$ is the equivalent strain, $\dot{\varepsilon}$ is the strain rate, $D_{i}$ is the initial grain size (before CDRX), $Q$ is the activation energy, $T$ is the absolute temperature and $C, k, j$ and $h$ are material constants. However, even if the grain size is referred as $D_{C D R X}$ by Valvi, this DRX approach may not be considered as a complete CDRX modelling. Indeed, the present model is close to several analytical approaches previously reported in literature in past years. We may cite the activity of Fratini and Buffa (2005) and their development of a model dedicated to the determination of grain size evolution induced by CDRX phenomenon in AA6082-T6 aluminium alloy during FSW process. The same research group (Buffa et al., 2007) extended the modelling to an AA7075-T6 aluminium grade two years later. Das (2010) proposed a similar power law evolution compared with the one previously used. This approach was also coupled with a multi-level cellular automata framework. Saluja et al. (2012) also developed a CAFE model to estimate grain size evolution in AA6061-T6 aluminium grades. Transition rules were applied depending from local values of temperature 
and strain field in order to estimate the associated grain size induced by CDRX mechanism in each CA cell. This value is reassigned at the larger FE scale in a second step.

Consequently, Valvi et al. (2016) also used the relations originally proposed by Fratini and Buffa (2005) to estimate the proper material coefficients. Interestingly, this latter activity was also applied both to similar (AA6061-T6) and dissimilar (AA6061-T6/AA50860) welding. The linear regression procedure originally applied by Fratini and Buffa (2005) to estimate grain size evolution in AA6082-T6 material leads to the following formula for an initial grain size $D_{0}$ :

$D_{C D R X}=40 \varepsilon^{-0.1648} \dot{\varepsilon}^{-0.322} D_{0}^{-0.104} \exp \left(-\frac{Q}{R T}\right)$

However the pre-exponential coefficient 100 as originally proposed by Fratini and Buffa for AA6082-T6 aluminium grade has been modified by Valvi et al. (2016) with a lower value of 40 probably due to the use of Al6061-T6 aluminium alloy corresponding to a change in the material of interest. Regarding their approach, the model of Valvi should be considered as an empirical model-based where calibration approaches are previously used to estimate unknown parameters. The final dislocation density distribution is evaluated after welding considering various transition rules. An initial relation (Eq. 44.1) is derived considering relationships between flow stress, dislocation density and grain size. In a second method (Eq. 44.2), the density of dislocation is simply estimated considering an empirical method based on a polynomial relation. The approach helps to capture the effect of stress and strain field onto the microstructure.

$\rho=\left(\frac{k}{\alpha_{0} E b}\right)^{2} \frac{1}{D_{C D R X}}$

$\rho=\sum_{k=0}^{4} p_{k} D_{C D R X}^{k}$

Various methods are used afterwards in order to predict the current true stress-strain relation and the associated formability of the FSW welded sheets to estimate the final mechanical properties of parts. The first method is based on the use of the initial behaviour law. The second method and third method are also based on the CAFE models previously described and also used in this second stage. However, in the third method, a reassignment strategy is applied. The overall true stress is indeed considered as the averaging of true stress in all CA cells of elements. In the last method, the current flow stress during tensile test has been evaluated. Relations with dislocation density evolution are proposed considering two models:

$\rho=\frac{4 \gamma}{b \lambda}$

$\rho=K_{1} \exp \left(-k_{1} \gamma\right)+K_{2} \exp \left(-k_{2} \gamma\right)+F_{S} / k_{2}$

where $\gamma$ is the plastic shear strain, $b$ the Burgers vector and $\lambda, K_{1}, K_{2}, k_{1}, k_{2}$ are model constant. The current flow stress is then estimated considering a simple linear relation depending from the square root of the dislocation density. 


\section{c. Results}

The grain size evolution has been computed based on Eq. (42) for various FSW experiments after a careful calibration to model dynamic recrystallization. As mentioned previously, models dedicated to DRX in FSW applications should only be limited to SZ. Indeed, no recrystallization mechanism occurs outside the path of the pin. However Valvi and co-authors have also considered that their results were relevant to estimate grain size evolution in TMAZ and HAZ domains. Some deviations were consequently observed by the authors between CAFE modelling and experiments in grain size estimations but limited to the TMAZ domain in similar welding (Fig. 33 a). In other domains, final predictions from CAFE models show evolutions of the order of experimental measurements even if some underestimations are reported. Analyses were also conducted on dissimilar welding (Fig. 33 b) and good agreement is obtained mainly close to the centre line. However, only the average grain size is provided by the authors. The decrease of grain size in the nugget domain when linear welding velocity increases is also observed as expected. As reported by Valvi et al. (2016), this phenomenon is experimentally observed and successfully modelled by the present CAFE approach. These results highlight the ability of CAFE approach to predict the features of microstructure.

(a)

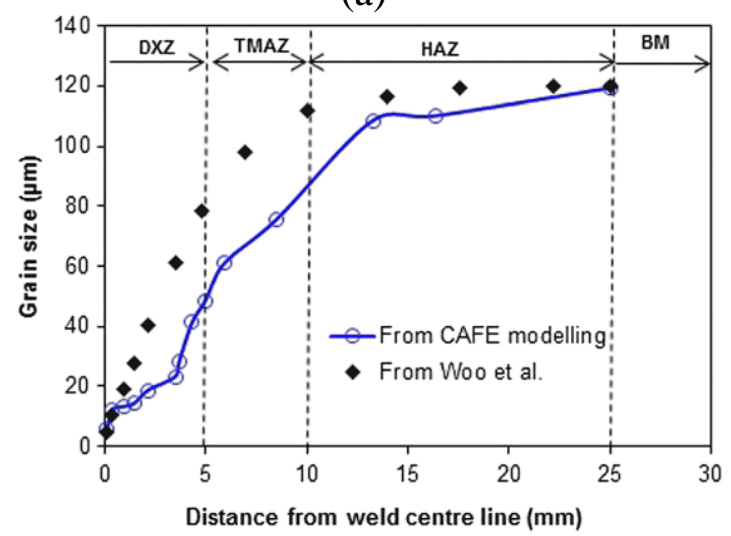

(b)

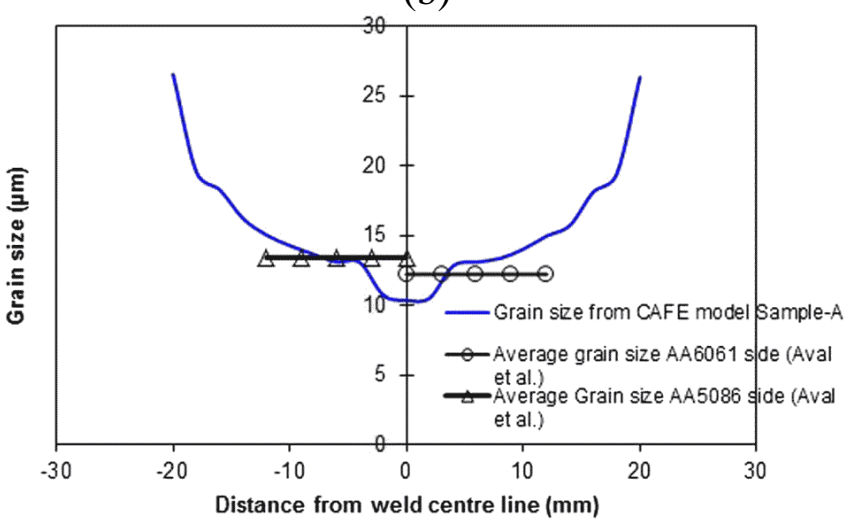

Fig. 33: Prediction and validation of grain size distribution during FSW of (a) similar Al grade combination when compared to experimental observation of Woo et al. (2008) and (b) dissimilar Al grade combination when compared on both side to experimental observations of Aval et al. (2011) (Valvi et al., 2016).

Valvi et al. (2016) estimate afterwards the dislocation density as based on the grain size distribution in the welded domain using analytical relation (Eq. 44.1) and empirical model (Eq. 44.2) after calibrations based on experimental data proposed by Woo et al. (2008). The predicted dislocation density also shows good agreement considering experimental data given by Woo for similar welding mainly in the TMAZ domain. However the authors noticed that 
analytical relation provides more accuracy results compared with empirical models. As grain size predicted with CAFE modelling depends from strain, strain rate and temperature (Eq. 42), CAFE model gives the unique opportunity to investigate influence of process conditions (velocity, rotation, geometry ...) on grain size and dislocation evolution through the welded domains. The authors considered that CAFE models prevent the use of many expensive experiments to investigate FSW process effects on microstructure evolution. These models give also the possibility to save time as the computation time are close to the one associated to single FE analysis developed on Abaqus ${ }^{\circledR}$ software. According to the authors, the main part of the activity developed to use relevant CAFE models correspond to the calibration stages, where accuracy experimental data are required. Indeed, calibration of CAFE models requires precise temperature evolutions as well as initial and final grain size in the welded domains. In addition relevant formulae as the one given in (Eq. 42-44) have also to be provided.

As a second stage, Valvi developed the four methods previously described to estimate mechanical behaviour of welded samples during tensile tests for similar and dissimilar FSW. These four methods are also validated considering data of Woo et al. (2008). Stress-strain predictions are well reproduced in all cases. However, the authors highlight that the fourth methods based on precise estimation of dislocation density (Eq. 45) provide better results. In conclusion, the authors considered that the CAFE predictions using a flow stress averaged on CA cells and based on dislocation density estimation are also accurate and reliable in the prediction of stress-strain evolution.

\section{- Physical model}

\section{a. Presentation}

The Gourdet and Montheillet model (GM) (2003) is the most widespread approach. This latter assumes that CDRX may result as the mixing between three elementary mechanisms corresponding to strain hardening, dynamic recovery and high-angle grain boundary (HAGB) migration. This model describes the polycrystalline structure through the distribution of the dislocation density in the joint and sub-joints during the deformation. A fraction of the dislocations created by the strain hardening groups together forming new sub-joints with very low disorientation angle $\left(\theta \approx 1^{\circ}\right)$. The remaining dislocations disappear in the grain boundaries or are absorbed into the existing sub-joints. In the latter case, the disorientation of the sub-joints increases progressively. They transform into joints when critical angle is reached $\left(\theta \approx 15^{\circ}\right)$. Grain boundaries are considered as mobile interfaces. In addition, an elimination mechanism is assumed when these boundaries encounter dislocations during migration. A part of the recovered dislocation participates to the development of new LAGBs (low-angle grain boundary). The other part is absorbed by the pre-existing boundaries. In addition, some of these dislocations are also suppressed by HAGBs. Simultaneously, those incorporated into LAGBs cause a progressive misorientation thus leading to HAGBs as explained by Huang and Logé (2016) (Fig. 34). 


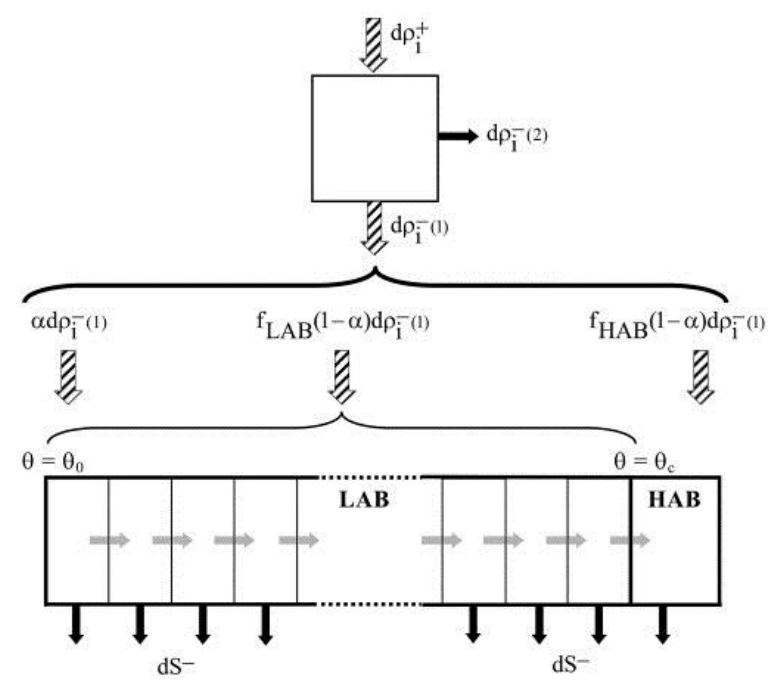

Fig. 34: Diagram showing the way dislocations developed by strain hardening are shared between microstructural elements (hatched arrows). Dislocations and boundaries absorption induced by migrating HAGBs is shown on black arrows. Grey arrows illustrate the progressive increase of low angle grain boundary (LAGB) misorientations from $\theta=\theta_{0}$ to $\theta=\theta_{c}$. LAB and HAB notations are similar to the HAGB and LAGB notations (Gourdet and Montheillet, 2003).

This GM model is based on the stress-strain curve. In addition, the evolution of the dislocation density inside crystallites is assumed to follow a modified Laasraoui-Jonas equation (Laasraoui and Jonas, 1991) provided by the following relation:

$d \rho_{i}=h d \varepsilon-r \rho_{i} d \varepsilon-\rho_{i} d V$

where $h$ and $r$, are respectively the strain hardening and the dynamic recovery of the material. The variation $d V$ corresponds to the volume swept by the moving grain joints. Considering a deformation increment $d \varepsilon$, a dislocation density $d \rho_{i}^{+}=h d \varepsilon$ is created by the strain (first part), while a dislocation density $d \rho_{i}^{-}=r \rho_{i} d \varepsilon$ is suppressed from the initial dislocation density $\rho_{i}$ by "condensation" in sub-grain or by "absorption" in pre-existing walls (second part). The part $d \rho_{i}^{-}=\rho_{i} d V$ corresponds to the annihilation of the dislocation located within the volume $d V$ swept by the moving grain joints (third part). For the sake of simplification, only the grain boundaries are considered as mobile, the movement of the sub-joints being sufficiently low to be neglected.

The GM model is able to forecast the evolution of crystallite size, dislocation density and equivalent Von Mises stress of the material respectively by the following equations:

$d S=d S^{+}-d S^{-}$

where:

$d S^{+}=\frac{b}{2 \theta_{0}} \alpha r \rho_{i} d \varepsilon$ 
$d S^{-}=f_{i} S^{2} v_{i} \frac{d \varepsilon}{\dot{\varepsilon}}$

which represents respectively the area of sub-grain boundary, $d S^{+}$, created during an increment in deformation and the area, $d S^{-}$, removed by the movement of grain boundaries. $b$ is the length of the Burgers vector, $\theta_{0}$ is the initial sub-grain boundary orientation. $\alpha$ is a parameter depending from the grain size, $D$. The number of dislocations families in a sub grain boundary is set between 1 and 3 according to Amelinckx and Dekeyser (1959) and chosen at the average value of 2 . Indeed, if the crystals are very large, the dislocations mainly group together to create new grain boundaries and only a few of them will be absorbed within existing boundaries. Conversely, if the crystals are very fine, their walls absorb a very large number of dislocations and few new grains disappear. The Von Mises equivalent stress of the material, $\bar{\sigma}$, is directly linked to the microstructural parameters and depends mainly on dislocation density $\rho_{i}$ within the grain as clearly established by Castro-Fernáandez and Sellars (1989) leading to the formula:

$\bar{\sigma}=M G b \sqrt{\rho_{i}}$

where $M$ is a constant close to the unity and $G$, the Young modulus of the material. Assuming that the parameters $f_{i}, v_{i}$ and $D$ are constant - i.e. at a sufficiently high deformation - the particular stationary solution of the differential equation would be of the type:

$\rho_{i}=\frac{h}{r+2 \frac{f_{i} v_{i}}{\dot{\varepsilon} D}}+\rho_{0}$

\section{b. Methodology}

This approach has been thereafter applied by Jacquin (2009) to model grain structure recrystallization during FSW processes with associated hypotheses. The material is considered as a set of grains without sub-grain boundary $\left(f_{i}=1, \rho_{i}=0\right)$ in the initial state. In the same state, the dislocation density in grains is close to the one reported in annealed metals $\left(\rho_{i}=0.01 \mu \mathrm{m} . \mu \mathrm{m}^{-3}\right)$ and the initial grain size are those initially measured. Strain rate and temperature influences are considered through the equations:

$G=G_{0}-G_{1} T$

$h=h_{0}\left(\frac{\dot{\varepsilon}}{\dot{\varepsilon}_{0}}\right)^{m} e^{\frac{m Q}{R T}}$

$r=r_{0}\left(\frac{\dot{\varepsilon}}{\dot{\varepsilon}_{0}}\right)^{-m} e^{-\frac{m Q}{R T}}$

$v_{i}=v_{0}\left(\frac{\dot{\varepsilon}}{\dot{\varepsilon}_{0}}\right)^{m^{\prime}}$

where $\dot{\varepsilon}_{0}=1 \mathrm{~s}^{-1}$. The constants introduced in these equations and following simulations are provided in table 5 . They have to be considered as intrinsic values for the material of interest. The expressions of $h$ and $r$ were originally estimated using stress-strain curves based on 
compression tests of 1200 aluminium grade as developed by Gourdet (1997). Gourdet and Montheillet (2002) have shown that dependence of grain boundary migration with temperature evolution is quite low when developing compression testing at large temperatures on polycrystalline aluminium samples. Montheillet and Le Coze (2002) also developed similar observations when investigating dynamic recrystallization mechanisms in high-purity metals. Indeed, on the one hand the driving force resulting from differences in local dislocation densities increases with $\dot{\varepsilon}$, while on the other hand the decrease in this driving force during temperature increase is almost entirely compensated by grain boundaries mobility increase. The $\alpha$ parameter is set to 0.1 , according to previous works performed by Chovet et al. (2000) on aluminium alloy of commercial purity.

In the particular case of steady state, Gourdet and Montheillet (2003) show that solving differential equations leads to an analytical solution. However, in the case of FSW, the material particles undergo a rise in temperature accompanied by significant deformations, followed by a relatively short cooling. Therefore, the steady state conditions cannot be applied. To do this, Gourdet and Montheillet have developed an iterative transient resolution method. The resolution program is based on the sequential calculation of the strain hardening and recovery parameters, dislocation density, grain boundary migration rate, disorientation and final grain size. These parameters depend on the time increment, temperature and $\dot{\varepsilon}$ value to follow a particle along its flow line. This model is based on the modified Laasraoui-Jonas equation. A particle, corresponding to an amount of material, is tracked during its deformation and heating cycle. The instantaneous strain rate, $\dot{\varepsilon}$, and corresponding temperature are evaluated at each time step. The GM approach has been applied and validated by Jacquin (2009) to model recrystallization mechanism in FSW process. Some EBSD measurements are performed on AA2024 samples obtained by FSW (Fig. 35). Different welding configurations have been investigated as detailed hereafter:

Case 1: cold welding, welding speed $400 \mathrm{~mm} \cdot \mathrm{min}^{-1}$ and rotational speed $400 \mathrm{rpm}$

Case 2: normal welding, welding speed $400 \mathrm{~mm} \cdot \mathrm{min}^{-1}$ and rotational speed $800 \mathrm{rpm}$

Case 3: hot welding, welding speed $200 \mathrm{~mm} \cdot \mathrm{min}^{-1}$ and rotational speed $800 \mathrm{rpm}$ 
(a) Case 1 - nugget

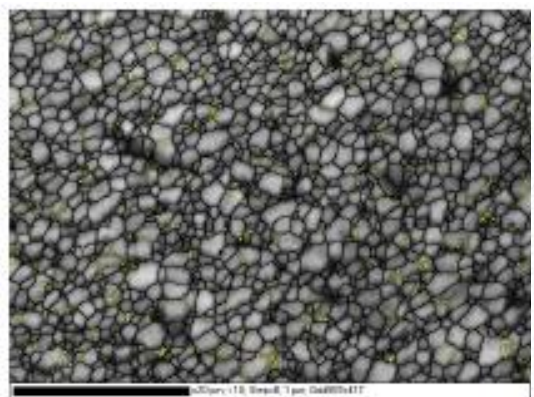

(b) Case 1 - flow arm (c) Case 2 - nugget

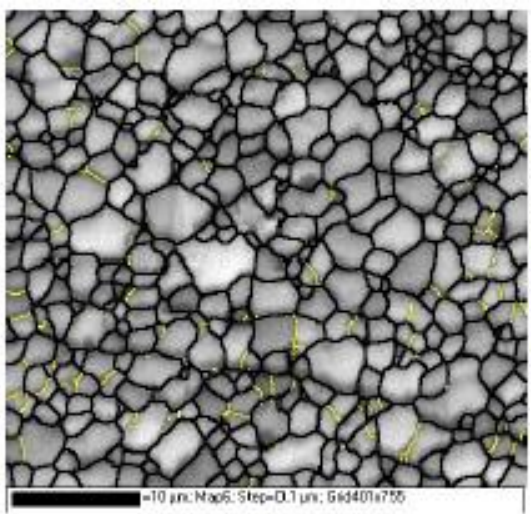

(d) Case 2 - flow arm

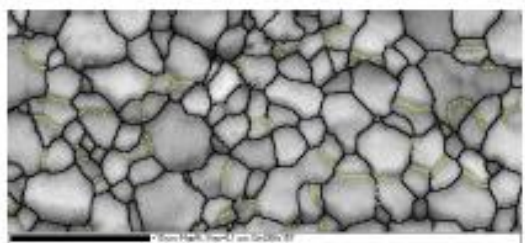

(e) Case 3 - nugget

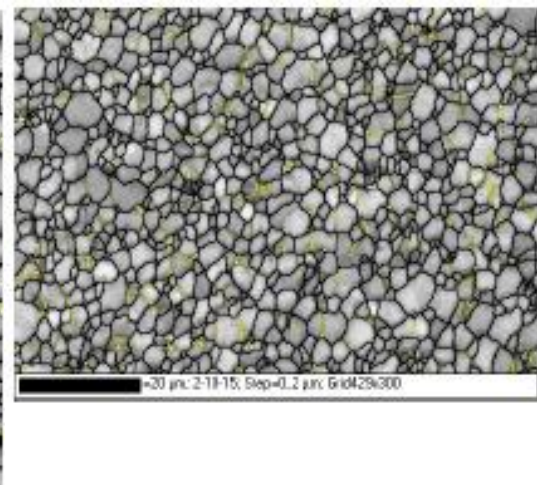

(f) Case 3 - flow arm

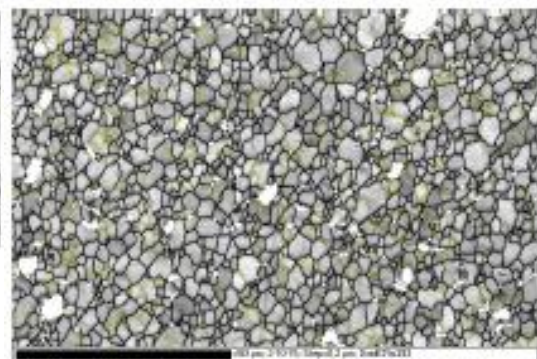

Fig. 35: EBSD cartographies of the nugget and flow arm in AA2024 samples (Jacquin, 2009)

The hardening or recovery parameters (Eq. 51-54) were determined experimentally or selected in the literature. The main parameters are summarized in table 5.

Table 5: Parameter used for the Continuous Dynamic Recrystallization (CDRX) modelling Gourdet-Montheillet approach. (Jacquin, 2009) on AA2024 aluminium alloy

\begin{tabular}{cccccc}
\hline \hline Name & $h_{0}\left[\mathrm{~m}^{-2}\right]$ & $r_{0}[-]$ & $v_{j}\left[\mu \mathrm{m} \cdot \mathrm{s}^{-1}\right]$ & $m[-]$ & $Q\left[{\left.\mathrm{~J} . \mathrm{mol}^{-1}\right]}^{12}\right.$ \\
\hline Data & $72.10^{12}$ & 1030 & 3 & 0.12 & 155000 \\
\hline \hline
\end{tabular}

Fig. 36 shows the thermomechanical history of a particle along its flow line, the starting point of this flow line being $3.5 \mathrm{~cm}$ upstream of the weld, at a distance of $1 \mathrm{~mm}$ from the joint line on the advancing side and at mid-thickness of the $3 \mathrm{~mm}$ thick sheet plates of AA2024 T351. The flow line is integrated on the basis of the velocity fields used in the thermomechanical model proposed by Jacquin (2009). The same velocity fields are applied to the thermomechanical model to obtain the corresponding temperature field. The welding conditions are selected in the list previously presented. This welding configuration was reproduced experimentally in order to compare and validate the microstructural model. 
(a)

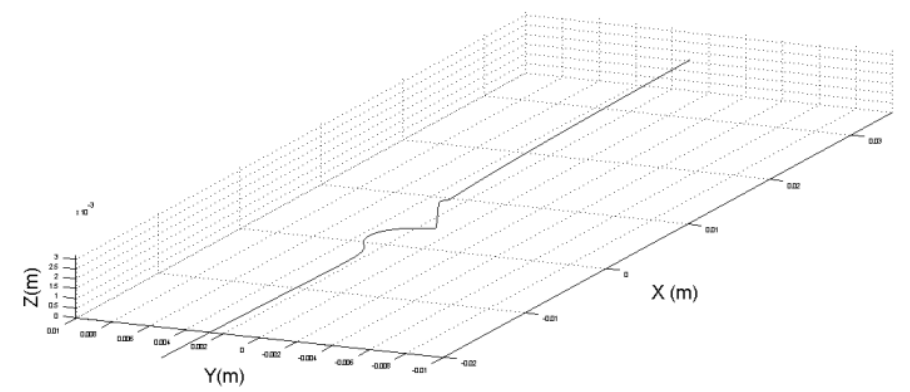

(b)

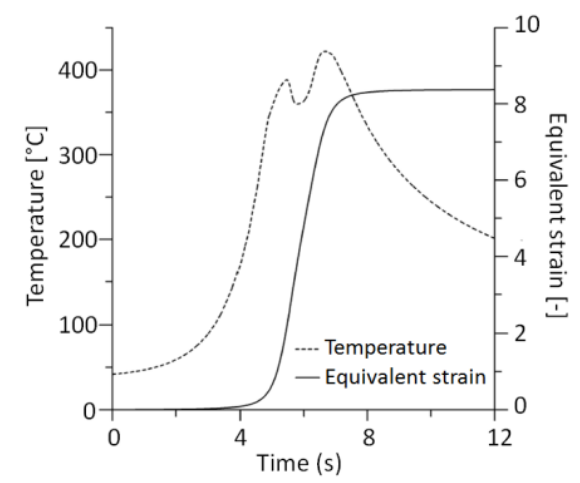

(c)

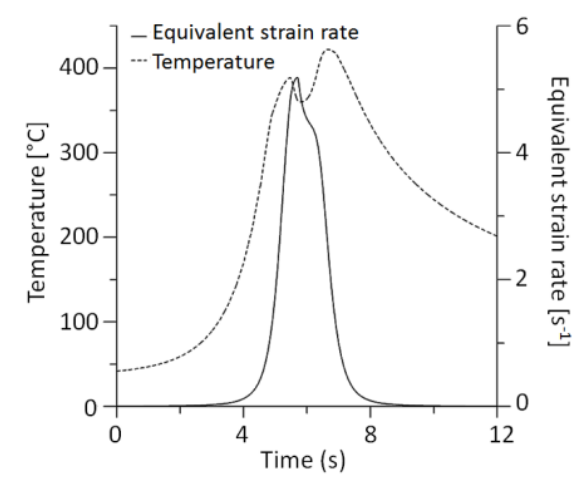

Fig. 36: Thermomechanical history endured by a volume of material for a traverse speed of $400 \mathrm{~m} \cdot \mathrm{min}^{-1}$ and a rotation rate of $800 \mathrm{rpm}$ (Jacquin, 2009).

\section{c. Results}

The simulation results are compared with experimental observations developed onto the nugget domain (SZ). Comparisons on grain size estimation are detailed on Table 6. Good agreement with experimental measurements is shown. The GM model coupled with a simple analytical thermomechanical model is able to provide a quick and relatively accurate estimation of the microstructural evolution during FSW. The main benefit of this approach is its small computation time of the order of a few minutes. In addition this coupled model can easily be reused for other alloys however undergoing CDRX phenomena. Moreover, such a model could conveniently be modified or adapted to other physical interaction. For instance the slowdown of grain joint migration by heterogeneous precipitation may be considered in future developments.

Table 6: Comparison of grain size between experiments and simulations in the nugget domain on AA2024 alloy (Jacquin, 2009).

\begin{tabular}{ccc}
\hline \hline Grain size & Measurement $[\mu \mathrm{m}]$ & Simulation $[\mu \mathrm{m}]$ \\
\hline Case 1 & 1.43 & 1.59 \\
Case 2 & 1.97 & 2.6 \\
Case 3 & 2.25 & 3.1 \\
\hline
\end{tabular}




\section{III.3.d. Monte-Carlo - Potts models}

\section{a. Presentation}

The Potts models derivate from the Monte Carlo (MC) techniques and are commonly designated with this latter expression. These methods are dedicated to the modelling of microstructure evolution induced by grain boundary movement during forming processes. These approaches were originally proposed by Potts (1952) in a primary work to model material evolution induced by temperature effect. Applications to these approaches in FSW modelling consequently aim at describing the change in grain texture map for a given temperature field during forming process. Literature reports several activities dedicated to the modelling of microstructure evolution in FSW processes based on Potts models and their application. Grujicic et al. (2015) carried out a thermo-mechanical model including microstructure evolution based on a Monte-Carlo simulation algorithm. The grain structure evolutions are predicted in the weld zones during the whole process including cooling stage. Temperature evolutions are computed using a finite-element method. The grain growth competition and recrystallization mechanism inducing grain refinement are consequently modelled considering local temperature fields. Zhang et al. (2016) also developed similar approach to model grain growth evolution in AA6082-T6 aluminium grade. Some particles were also monitored in the thermo-mechanical simulation to provide estimation of the stirring domain (TMAZ). The domain enduring grain growth evolution is similarly associated to the heat affected zone (HAZ). Zhang also emphasized the success of these methods and their efficiency to predict grain growth and topological features in many fields including welding, abnormal and anisotropic grain growth and evolutions of polycrystalline microstructures. It should also be noticed that this model are usually applied to model grain structure induced by recrystallization processes but also temperature field evolution leading to the associated texture evolution in BM, HAZ, TMAZ and SZ domains. This approach provides at the end a complete final state of the microstructure field induced by FSW process.

This approach also considers a grain microstructure description based on the same Cellular Automaton approach as previously described. A 2D computational region is selected in each weld zone of interest and divided into square cells. Domain sizes are of the order of $500 \mu \mathrm{m} \times$ $500 \mu \mathrm{m}$ and $\sim 10^{4}$ to $\sim 10^{5}$ cells are considered depending from computational capacity. A Voronoi-cell-type grain structure may be used to define the as-delivered material state as proposed by Grujicic et al. (2015) (Fig. 37). The grain orientations are randomly selected in each grain and associated to a set of integer values of the order of $\sim 100$. In addition, it should also be noted that periodic conditions are assumed at the domain boundaries. 
(a)

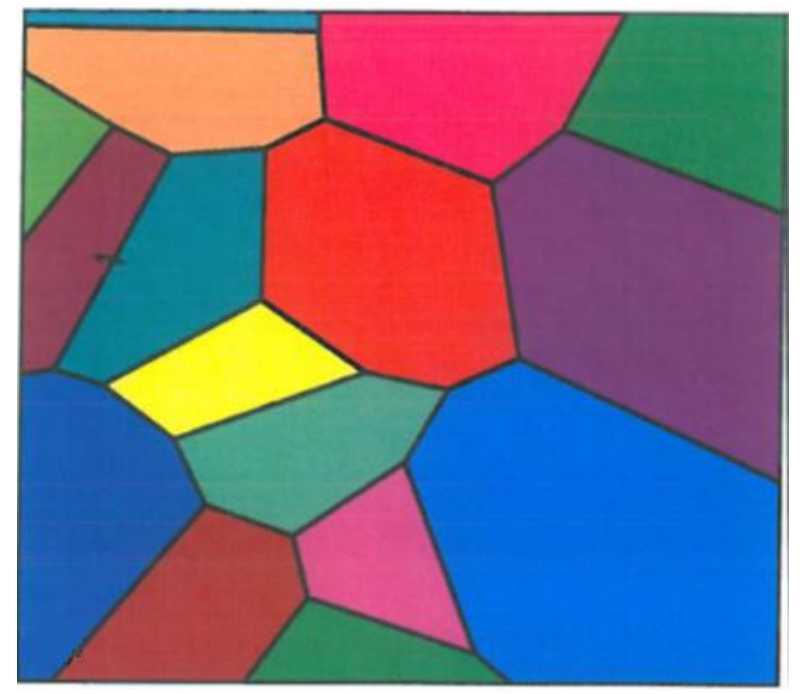

(b)

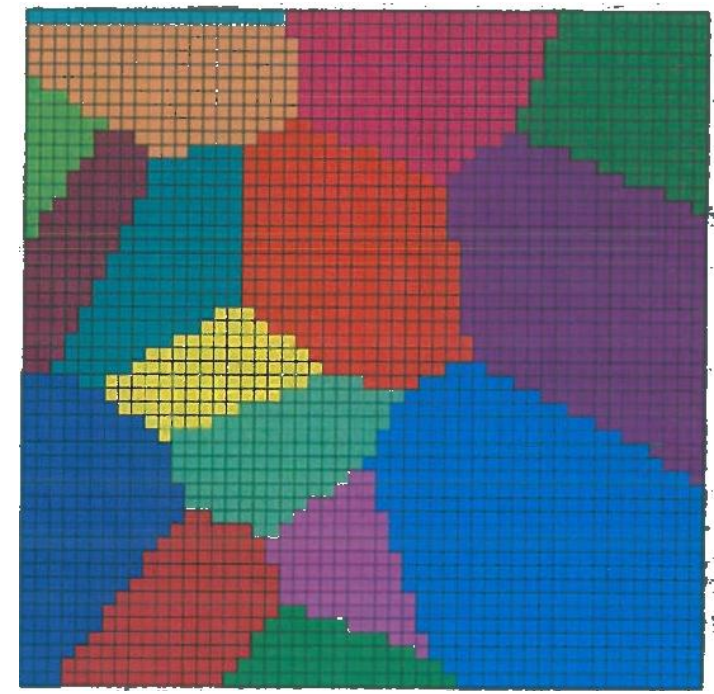

Fig. 37: (a) Schematics of the Voronoi-cell grain description as used by Grujicic to model initial microstructure in metal. Colours correspond to grain orientations. (b) Square cell discretized microstructure used in the MC simulation procedure. (Grujicic et al., 2015)

\section{b. Methodology}

The Potts model aims at decreasing the total energy, $E$, of the computational region when considering possible changes in cell orientation. Several expressions are reported in the literature in order to estimate this energy. Grujicic et al. (2015) propose the expression:

$E=\frac{1}{2} E_{G B} \frac{L^{2}}{n} \sum_{i=1}^{N} \sum_{j=1}^{n}\left(1-\delta_{q_{i} q_{j}}\right)+\frac{L^{3}}{n^{3 / 2}} \sum_{i=1}^{N} H_{v_{i}}$

where $N$ is the total number of cells, $L$ is the domain size, $n$ is the number of nearest neighbours for square cells (i.e. 8 in 2D simulations), $E_{G B}$ is the homogeneous grain boundary energy associated to cells and $H_{v_{i}}$ is the strain energy of the same cells $i$. Consequently, expression (55) contains both the energy contributions associated to grain boundaries and to strain. These two contributions help hereafter to simulate microstructure evolution induced by grain growth and dynamic recrystallization stages. Zhang et al. (2016) simplify this expression afterwards neglecting the second terms and modelling separately the grain nucleation induced by DRX. The effect of energy change on microstructure evolution is done considering the possibility for each cell to be captured by one of its neighbours and adopt the same orientation consequently. Cell changes are also assumed during Monte-Carlo step which differ from time step used for thermal resolution. Considering Eq. (55), the variation in total energy, $\Delta E$, lead to two possibilities for the computation of transition probability (Eq. 56).

$P_{\text {growth }}=\left\{\begin{array}{c}1 \text { if } \Delta E<0 \\ e^{-\frac{\Delta E}{k_{B} T}} \text { if } \Delta E>0\end{array}\right.$ 
where $T$ is the instantaneous temperature of cell and $k_{B}$ is the Boltzmann constant. Thus, an energy decrease will lead to a transition acceptation (first case in Eq. 56). In the opposite, if energy increases, a number, $R$, is randomly generated in the domain [0:1]. The transition acceptation is only possible if $R<P_{\text {growth }}$ leading to a possible change in cell orientation even if the total energy increases (second case in Eq. 56). However, the probability of transition obviously decreases for largest energy variations. The transition is also restricted to boundary cells having a neighbour cell with another orientation. Grain growth is then the consequence of changes in cell state at the grain boundaries. In addition, Grujicic and Zhang also consider the possibility to nucleate new grains in the CA lattice depending from the local temperature. Grujicic et al. (2015) add strain energy, $H_{v_{i}}$, dependence on nucleation processes whereas Zhang et al. (2016) include equivalent strain rate, $\dot{\bar{\epsilon}}$, effect computed at the macro scale. This step mimics the possibility to develop new grains by fragmentation during the recrystallization mechanism. The associated probabilities of nucleation are thus given by the following expression considering both effects:

$$
\left\{\begin{array}{c}
P_{n u c l}=1-e^{-\frac{L^{3} H_{v}}{n^{3 / 2} k_{B} T}} \\
\dot{n}=N_{0} \dot{\bar{\epsilon}} e^{-\frac{Q}{R T}}
\end{array}\right.
$$

where $N_{0}$ is a nucleation constant and $Q$ is an activation energy. The approach gives the possibility to introduce new grains in the lattice as a probability of nucleation occurrence, $P_{\text {nucl }}$, or corresponding to a specific nucleation rate, $\dot{n}$. In addition, the nucleation rate is not constant during FSW process as an increase in temperature or strain rate leads to the development of new nuclei. However the drawbacks of such model is the need to link effective process times, $t$, also used in the process simulation and MC steps , MCS, required to model cell state changes. Consequently, the relationship between the number of MCS discrete time steps and the real process time, $t$, has to be established. This latter relation thereafter provides an estimation of the number of MCS required to achieve the expected microstructure feature. Grujicic et al. (2015) propose to apply the following relation (Eq. 58). Zhang et al. (2016) provide similar relation even if their expression of grain boundary energy shows some differences.

$\operatorname{MCS}^{(n+1) n_{1}}=\left(\frac{D_{0}}{K_{1} L \sqrt{n}}\right)^{n+1}+\frac{(m+1) \alpha C_{1}{ }^{m}}{\left(K_{1} L \sqrt{n}\right)^{m+1}} \sum_{i}\left[\exp ^{m}\left(-\frac{Q}{R_{U} T_{i}}\right) t_{i}\right]$

These models require considering the initial features of microstructure to model grain structure evolution. It should be pointed out that MC models are isotropic approaches where grain structure evolutions are similar in all the dimensions. Deformation-induced grain distortions are not modelled. To overcome this difficulty, Grujicic and Zhang propose to consider anisotropic grain structure at the onset of MC simulation in TMAZ and SZ where elongated grains are initially present. This non-equiaxed structure is developed with the same average grain size than the equiaxed HAZ. However an aspect ratio proportional to the 
principal components of the local plastic strain is considered by Grujicic et al. (2015). Zhang et al. (2016) develop similar approach whereas considering traced particles introduced in FE simulations and their average elongation ratio. Consequently the texture in grain shape in initial state shows dissimilar orientation in domains directly affected by stirring process.

\section{c. Results}

Fig. 38 (Grujicic et al., 2015) shows the grain structure evolution onto the AA5083-H321 alloy after FSW process in the three domains of interest. Process parameters correspond to a rotation of $500 \mathrm{rpm}$ and a traverse speed of $2.5 \mathrm{~mm} . \mathrm{s}^{-1}$ with a tool diameter of $18 \mathrm{~mm}$. The initial grain size is assumed as equal to $20 \mu \mathrm{m}$. The maximal temperatures reported by the authors during FE simulations are respectively equal to $710 \mathrm{~K}, 815 \mathrm{~K}$ and $900 \mathrm{~K}$. This figure demonstrates the effect of FSW process onto the grain evolution. In HAZ (Fig. 38 a), a noticeable grain growth is reported by the authors with a final grain size from $20 \mu \mathrm{m}$ to $32 \mu \mathrm{m}$ with a preserved equiaxed feature. In TMAZ (Fig. $38 \mathrm{~b}$ ), a maximal strain of the order of 2.1 is achieved. Even if the initial structure was not equiaxed, the grain aspect ratio decreases toward unity. However simulation shows a final slightly non-equiaxed microstructure and an extent in grain growth to $\sim 38 \mu \mathrm{m}$ compared to HAZ. This evolution is mainly explained by the higher temperatures endured by material. In SZ (Fig. 38 c), computations show a change in microstructure evolution depending from the vertical position in weld centre line. High equivalent plastic strains are encountered with values as large as 21 . In addition, numbers of dynamic recrystallization processes (57) are achieved. The upper domain also experiences slightly higher temperature and subsequently larger temperature compared to bottom part of the SZ. This leads to a finer grain structure in end-state in upper part $(\sim 13 \mu \mathrm{m})$ compared to the bottom part $(\sim 17 \mu \mathrm{m})$ mainly explained by the dynamic recrystallization mechanism. As assumed by Grujicic, no direct experiment has been developed to validate these results and grain size prediction. However, comparisons of the present model with another model provided by Fratini et al. (2009) on a 2139-T8 butt joint show similar grain size profile.
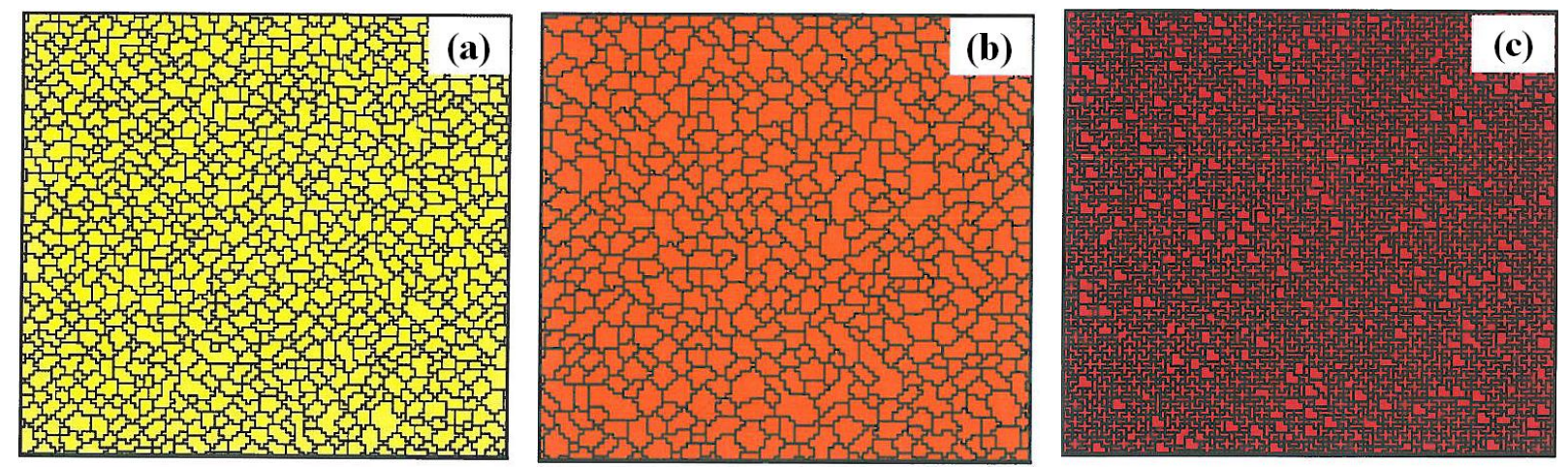

Fig. 38: As-FSWed grain microstructure associated respectively with the (a) HAZ, (b) TMAZ (retreating side) and (c) SZ (upper domain in centre line) at points located inside the material (Grujicic et al., 2015). 
Zhang et al. (2016) also apply same MC model in order to follow microstructure evolutions in FSW processes in AA6082-T6 alloy, even if some slight differences are observed between the two approaches in different steps of the algorithm. Fig. 39 shows the grain structure evolution in three cases of interest corresponding to changes in process parameters. The traverse speed was chosen to $100 \mathrm{~mm} \cdot \mathrm{min}^{-1}$ and pin diameter is equal to $3 \mathrm{~mm}$. Case \#1 (Fig. 39 a) corresponds to a rotation speed of $1000 \mathrm{r} \cdot \mathrm{min}^{-1}$ and a shoulder diameter of $10 \mathrm{~mm}$. Case \#2 (Fig. 39 b) and case \#3 (Fig. 39 c) investigate, respectively, the effect of increased rotation speed $\left(1500 \mathrm{r} . \mathrm{min}^{-1}\right)$ and increased shoulder diameter $(13 \mathrm{~mm})$ compared to case \#1. As shown by Zhang, the grain size substantially increases in each simulation compared to initial state, which is an opposite evolution compared to the previous results obtained by Grujicic (Fig. 38 c). However, Zhang considered that this evolution is mainly influenced by temperature evolution endured by material in this simulation. In addition smaller grain size is used in initial state. Dynamic recrystallization phenomenon happens during the early stages at high temperature leading to a mixture of small nuclei and large grains. At lower temperature, this population of grains undergoes the same evolution when DRX phenomenon is negligible. A final equiaxed structure is observed with some discrepancies in grain size. Comparison of Fig. 39 (b-c) with Fig. 39 (a) demonstrates a clear effect of the change in rotation speed or pin diameter when comparing final grain size. Indeed, an increase in each parameter leads to larger grains. In addition, these parameters tend also to extend the width of welding zones as reported by Zhang et al. (2016).

(a)

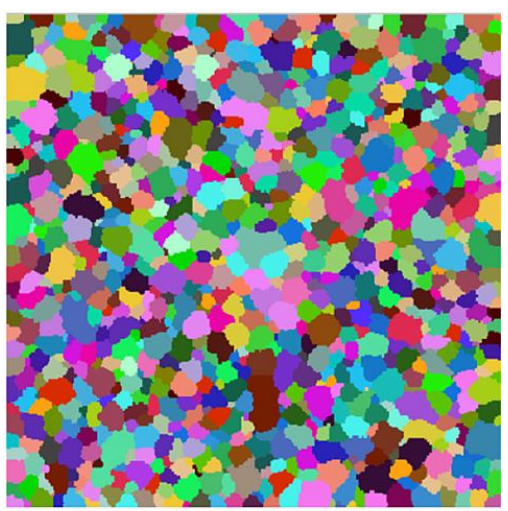

$163 \mathrm{MCS}, \mathrm{L}=17.6 \mu \mathrm{m}$ (b)

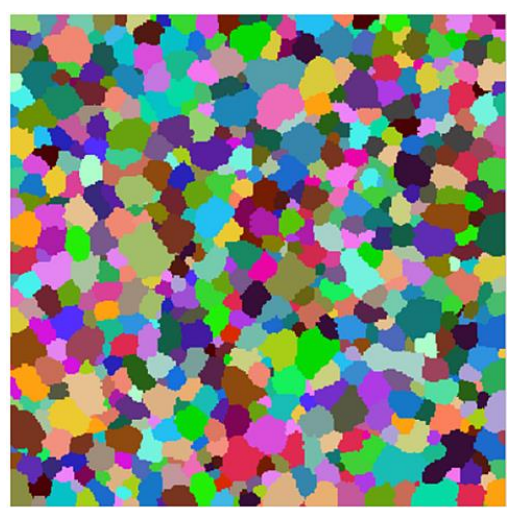

$230 \mathrm{MCS}, \mathrm{L}=18.9 \mu \mathrm{m}$ (c)

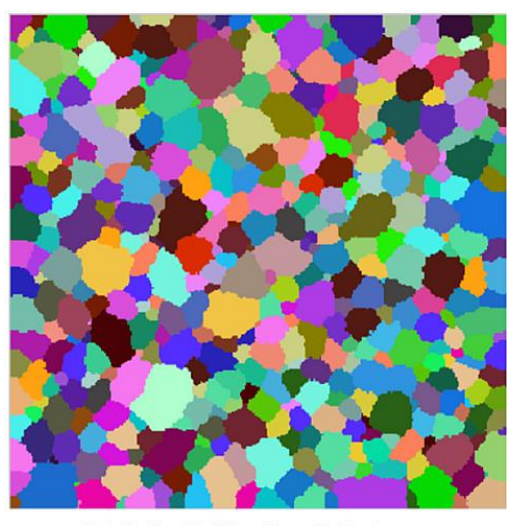

$283 \mathrm{MCS}, \mathrm{L}=20.8 \mu \mathrm{m}$

Fig. 39: Microstructure of grain growth in case \#1 (a), \#2 (b) and \#3 (c) investigated on the top surface (1 mm from welding steam) in the SZ domain. Monte-Carlo time-step, MCS, and final grain size, $\mathrm{L}$, are reported in end-state after cooling. The initial grain size was equal to $5.4 \mu \mathrm{m}$ in each simulation (Zhang et al., 2016). 

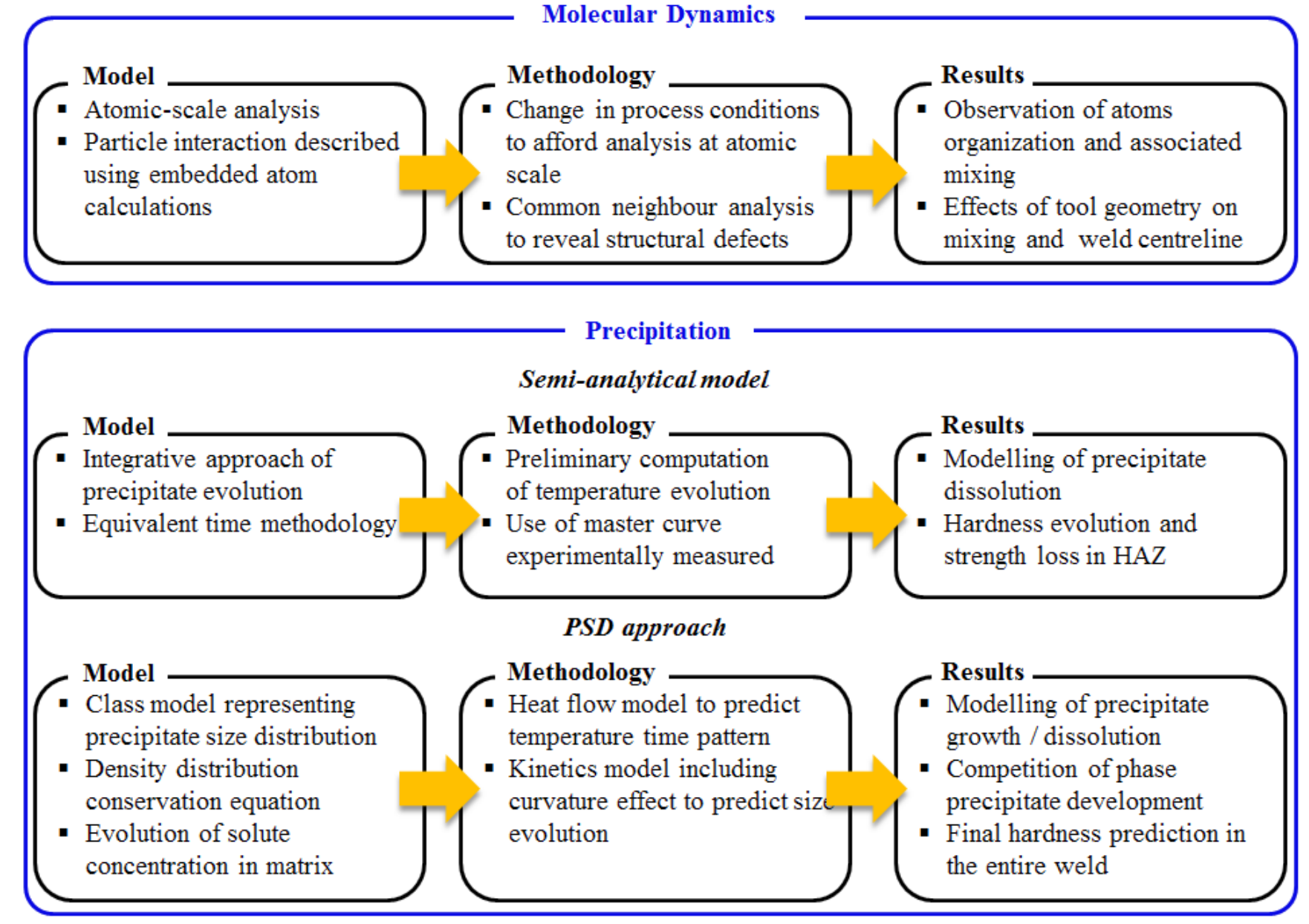

Fig. 40: Flow chart summarizing the characteristics, methodologies and results associated to molecular dynamics and precipitations models. 


\section{Recrystallization}

\section{DDRX}

Model Methodology

- Based on temperature and

strain evolution

- Derby-Ashby model

- Zener-Hollomon model

Evolution according to the grain

boundary mobility and the store

work energy in subgrain walls

Results

- Avrami model

- Final grain size depending from the strain rate via a power law

\section{Model GDRX \\ Methodology}

- Mrangnell and Heason model

Subgrain size computation

depending from critical

temperature and strain rate

values

- Grain growth evolution

- Final grain size

- Volume fraction of dynamic

recrystallized grain

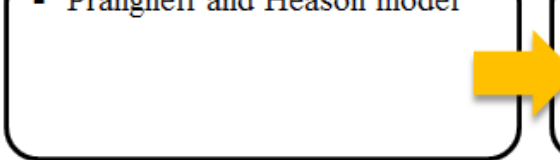

Model

\section{CDRX}

- Cellular Automaton - Finite

Element approach

- Lenard power law

- Physical model

Gourdet-Montheillet

\section{Methodology}

Heat conservation equation

solution

Results

Dislocation density analysis

regarding the dynamic recovery and strain hardening effect

\section{Monte-Carlo}

Model

- Grid of square cells

- Transition rule depending

from neighbourhood

- Computation of the decrease in total energy

- Instantaneous grain size reducing

Post dynamic grain growth

- Cell state evolution mimics grain boundary evolution

- Probabilistic approach

- Boundary movement simulation

Results

- Grain size evolution

- Limited to the stirred zone

- Temperature field

- Low computing time

Results

- Texture map depending from welded domains

- Grain size evolution

- Process parameters effect

Fig. 41: Flow chart summarizing the characteristics, methodologies and results associated to recrystallization models. 


\section{Recommendations}

A state of the art has been provided in previous chapter on the current developments in the field of microstructure modelling in aluminium alloys during FSW processes. Results reported in literature for various aluminium alloys and different FSW parameters have been investigated. In addition, in order to highlight the main features of models reported in present analysis, these latter are summarized in Fig 40 and Fig. 41. These two figures are respectively associated to i) molecular dynamics and precipitation models (Fig 40) and ii) recrystallization models (Fig 41). Both figures summarize the models features, their methodology and the final associated results. These figures should consequently help the discussion developed hereafter in present part. Indeed, as discussed previously, the literature review shows that most sources focus either on precipitation or grain size change phenomena also considering sources dedicated to both phenomena as Dos Santos et al. (2018). The investigation of the grain size evolution is restricted to the weld stir zone, whereas the precipitation investigations are more generally extended to the whole weld. Indeed the phenomena of dynamic recrystallization (DRX) are directly linked to the complex thermomechanical history undergone by material within the stir zone whereas the precipitation evolution is mainly induced by thermal evolutions and the initial microstructural state of the material. In practice, the nucleation of new grains will take place, in a privileged manner, on the sites of point and line defects such as dislocations and vacancies. Initial knowledge on the concentration of these defects and their evolution depending from temperature evolution will therefore be essential to propose a relevant modelling of precipitations processes.

The mastering of the grain size and precipitation states in the joined material after FSW process is essential to predict end-use properties. Indeed, these two quantities have large effects on the in-service strength of the welds. For example, the precipitation state may help to determine material toughness, or the occurrence of cracking mechanisms. The $\mathrm{W}$ shape of the hardness profile may also be estimated showing the magnitude of inhomogeneity in hardness evolution. Similarly, the grain size is relevant to investigate corrosion resistance of welded part, tensile strength or fatigue properties as mentioned by Zhang et al. (2016). However, the kinetic of the material changes occurring during FSW is still insufficiently considered in literature. These changes correspond to discrepancies induced by FSW between initial and final material properties, which may in some cases result in non-compliance or dramatic inservice strength defects.

\section{IV.1. Precipitation modelling}

\section{a. Thermodynamic coupling}

The more advanced models dedicated to precipitation mechanism are currently based on PSD approach as detailed previously in Table 2. As presented, simulations can be currently achieved to estimate precipitates evolutions induced in both heating and cooling stages. In 
such models, some recent benefits correspond to the development of thermodynamic couplings which has provided better prediction of particles evolution. These developments are based on the computation of both thermodynamic equilibrium at the precipitate/matrix interface and local values of chemical diffusion coefficients as proposed by Thermo-Calc software (2019) and applied by Legrand et al. (2015b). Such thermodynamic coupling should be encouraged in the modelling of microstructure evolution in FSW processes in order to improve the estimation of precipitate growth velocity in multicomponent alloys for industrial applications. Two softwares are currently used by FSW researchers for coupling applications, JMatPro and Thermocalc, both based on the CALPHAD (2019) approach and aluminium thermodynamic database. Dos Santos et al. (2018) have recently used the first software to model precipitation processes on AA7449 grade while Legrand et al. (2015b) developed application based on the second one for AA2024 grade. However to our knowledge no benchmark has been reported in order to compare these tools, thermodynamic databases or PSD numerical strategies proposed in the literature. This type of comparisons may benefit to establish relevant strategy in the modelling of precipitate evolution in FSW.

In addition, a better knowledge and mastering of diffusion process would also be promoted by the consideration of cross-diffusion phenomena in the estimation of precipitate growth kinetics in PSD modelling. This point has focused few interests of researchers despite the demonstration of its large effect on precipitate growth when metallic alloys endure heat treatment. Indeed, Rougier et al. (2013) have investigated cross-diffusion effect on precipitate development in nickel based superalloys demonstrating its clear influence on growth kinetics. The large number of added elements in current industrial aluminium alloys may also increase this cross-diffusion effect on precipitate growth kinetics. A better knowledge of mobility properties and improvement of associated database should also promote a better description of diffusion matrices also considering local temperature and composition effects. In addition short-circuit mechanisms promote diffusion processes in stirred material and are certainly involved in precipitate growth mechanisms. This diffusion mechanism has been mentioned by Frigaard et al. (2001) as an explanation of the increase in dissolution kinetics experimentally observed when discussing hardness measurements. According to the authors, plastically deformed materials should respond quicker to reheating compared to undeformed materials. Indeed the dislocations entangled within the microstructure act as path of high solute diffusivity (i.e. 'short circuit'). This diffusion mechanism is still largely difficult to estimate and only empirical corrections are proposed as done by Frigaard et al. (2001). However we have also to overcome in future developments some other current limitations encountered in the application of PSD models.

\section{b. Growth/Dissolution kinetics estimation}

Indeed, precipitates shape are usually assumed as regular sphere and few developments are provided on anisotropic shapes and anisotropic growth. However, we may cite the work of Gallais et al. (2008) dedicated to cylindrical shaped precipitates. Kamp et al. (2006) 
considered the development of plate shaped precipitates. Similarly, Dos Santos et al. (2018) investigated the nucleation and growth of plate precipitates on grain boundaries. Nevertheless, future applications will have to consider more realistic precipitate shapes as reported in TEM observations to provide better estimation of growth and dissolution kinetics. Indeed, Chen et al. (2009) developed TEM observations on AA2219-T6 aluminium grade showing plateshaped $\theta^{\prime}$ and $\theta^{\prime \prime}$ strengthening precipitates corresponding to metastable phase in base metal evolving toward more stable $\theta$ precipitate in the nugget. Sauvage et al. (2008) observed fine $\beta^{\prime \prime}$ needle-shaped hardening precipitates growing along <001> direction in Al-Mg-Si alloy after FSW. Dos Santos et al. (2018) reported observations showing a set of larger plate shaped precipitates in TMAZ corresponding to $\eta$-phase on AA7449-TAF grade after STEM characterization. Phase field simulation could consequently help to determine precipitate shape and favourable kinetic orientations in order to improve growth laws used at a larger scale to determine precipitate size distribution evolution. Such simulations at the micro-scale could also help to improve knowledge on nucleation mechanism and nucleation rate where few theoretical developments have been provided in FSW studies. In this way a better estimation of nucleation Gibbs free energy would be provided by thermodynamic coupling. In addition, growth kinetics models usually rely on Laplace approximation assuming no effect of composition field evolution. Even if this approach is validated at low oversaturation, large discrepancy occurs when oversaturation is higher than 0.1 leading to an underestimation of precipitate kinetics as shown by Legrand (2015a). The analytical solution and mathematical developments of Aaron et al. (1970) provides exact estimation of precipitate kinetics for any oversaturation and should be applied. In addition, these developments can be extended to other geometry also including cross diffusion phenomena.

We have also to mention that no clear detail on precipitate evolution in dissimilar welding have been reported in previous chapters. Indeed, literature review seldom report simulations of precipitate evolution when two aluminium grades or even metallic alloys are considered despite the large interest of dissimilar welding as investigated by Hamilton et al. (2019). In a recent review article, Patel et al. (2019) highlight also the advantages, difficulties and challenges associated to dissimilar aluminium welding in FSW processes. A large discussion is proposed regarding process parameter influence, tool positioning and tool geometry. Microstructural evolutions were also considered as well as obtained hardnesses and tensile strength properties in an in-depth discussion on dissimilar aluminium combinations.

However, we may cite among the few studies reported on dissimilar aluminium welding, the activity of Hamilton et al. (2016). This latter is dedicated to the dissimilar aluminium welding of 2017A-T451 and 7075-T651 alloys and to the modelling of temperature and material flow evolution with Comsol multiphysics software (Comsol, 2019). Alternate layers of 2017A and 7075 grades were observed experimentally with unique temperature history and associated precipitate distributions. The authors correlate simulated temperature and flow evolutions to microstructure and hardness properties. Reprecipitation of GP zones during cooling were observed in both alloys. However, clear differences were also observed and discussed by authors regarding hardness evolution. Indeed, progressive decrease in hardness 
from the core position is observed on 2017A alloy despite more constant values obtained on the side corresponding to 7075 alloy. Preliminary research activities have also been recently developed by Gopkalo et al. (2019). This latter is dedicated to the modelling of precipitates evolution during dissimilar welding of Al-Mg-Zn and Al-Mg-Si alloys. The Shercliff-Ashby model was used and fitted to the ageing kinetics evolutions deduced from hardness measurements developed after isothermal treatments separately applied on each alloy. An analytical non-isothermal model based on equivalent time was simultaneously developed to describe thermal evolution during process. These models were applied later on to estimate final mechanical properties of the dissimilar welded pieces. According to the authors, the combination of the Shercliff-Ashby model and the thermal model provides correct estimation of hardness evolution across welds. In addition, the minimum hardness position is estimated with less than $15 \%$ of difference compared to measurement positions.

Despite these recent activities, future developments are still required to provide relevant estimation of entire end-use properties in dissimilar welding and to cover a large range of aluminium alloys as an answer to industries needs in FSW simulations. More generally, the simulations of the mixing of chemical elements in FSW and local estimation of chemical compositions when various metallic grades are involved in joining process has created apparently few interest in the field of precipitate modelling. Some difficulties in such modelling may also arise from the large set of phases that may form in such conditions with complex interactions. In addition, the scale of solute element mixing introduced by FSW process should also be considered to clarify its importance and related effects.

\section{c. $\quad$ Numerical development}

Numerical improvements should similarly be promoted to follow precipitate evolutions in PSD modelling on aluminium alloys. Indeed, Eulerian approaches have been used in all PSD models reported previously (Table 2) to investigate precipitate evolutions during FSW process following the original development of Myhr and Grong (2000). However, this Eulerian approach is known to lead to scattering and spreading phenomena in precipitate size distribution for numerical reasons. A Lagrangian approach as initially proposed and detailed by Perez et al. (2008) would be beneficial in future development. Better understanding of precipitate evolution would be expected even if specific numerical implementation should be assumed in this context. Benchmark may also help to validate numerical development considering concurrent developments done by several researchers. In this aim a call to benchmark could be an opportunity to compare and propose relevant modelling strategies to follow precipitate distribution in multicomponent and multiphase alloys also considering results provided by commercial software as TC PRISMA (2019).

At a lower scale, numerous approaches are available in literature to follow precipitate evolution based on molecular dynamics or Monte-Carlo model. Some developments have been proposed to apply these models to FSW process as the activities previously detailed of Dmitriev et al. (2014), Nikonov et al. (2015) and Nikonov et al. (2016) in molecular 
dynamics. However this modelling is limited considering the complexity of stirring processes and coupling of physical phenomena in FSW. In addition, the polycrystal grain feature of industrial alloys may lead to discussion on the limitation of such modelling.

In addition to these developments dedicated to the tracking of precipitate size distribution and estimation of its associated effects, some authors have recently developed indirect numerical strategies of interest. In corrosion science, response surface method (RSM) has been used to obtain direct estimation of final corrosion resistance of joints in AA2219 aluminium alloy induced by precipitation process as developed by Rambabu et al. (2015) however without consideration of full precipitation process.

\section{d. Experimental evaluation}

The experimental development dedicated to direct observation of precipitation mechanism in FSW are of clear interest in order to provide direct estimation of precipitate evolution in the various welded domains. Few activities in this field are reported due to the complexity of such observations. Consequently the recent work of dos Santos et al. (2018) is of prime interest and corresponds to a breakthrough in the development of precipitate evolution. The authors have developed a welding experiment (Fig. 42 a) using the high energy beam line HARWI II of the Helmholtz-Zentrum Geesthacht. This latter was located at the former DORIS III synchrotron storage ring at the Deutsches Elektronen-Synchrotron (DESY) research centre in Hamburg, Germany.

(a)

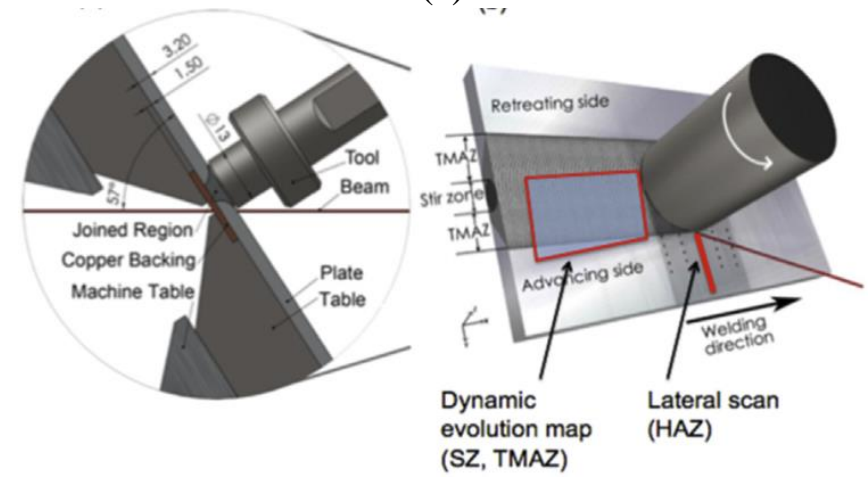

(b)

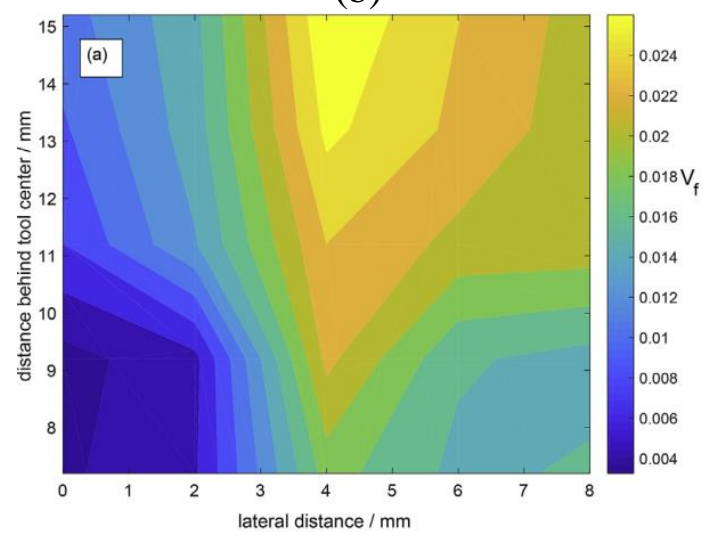

Fig. 42: (a) Set up of FlexiStir system showing side view (left) and isometric view (right). Regions of direct analyse (dynamic precipitate evolution) are highlighted. Two domains of interest are visible behind the tool (red rectangle) and on lateral scan in the HAZ domain (red line). (b) Map showing the measured evolution of precipitate volume fraction behind the tool. (Dos Santos et al. 2018).

In this experiment the $3.2 \mathrm{~mm}$-thick sample is moved under the rotating tool with various velocities. A tilt angle of $57^{\circ}$ is used such that the X-ray beam passes next to the tool shoulder. The analysed domains are fixed and their fraction of precipitates is measured based on SAXS (Small angle X-ray scattering) method. In this approach, the scattering signal 
measured after subtraction of the backing sheet is integrated along the azimuth to provide access to the scattering curve. This curve is afterwards fitted based on a two phase model of precipitates dispersed in the matrix considered as a homogenous domain. This fitting give access to both the total volume fraction of precipitate and the mean particle radius in each analysed volume corresponding to resolution size of $150 \mu \mathrm{m}$ on the sample surface (Fig. 42 b). Detectable precipitate sizes are restricted to the domain of 0 to $20 \mathrm{~nm}$ in this SAXS configuration. In addition no direct measurement under the tool is possible even if this domain is the one of main interest.

In-depth results were obtained with this equipment. Fig. 43 a) shows the volume fraction measured on the lateral scan developed (red line on Fig. 42 a). The authors demonstrated that the lowest volume fraction is close to the shoulder with a large decrease of $65 \sim 85 \%$ of volume fraction of precipitate compared with the initial value of $4.7 \%$ in precipitate volume fraction. However this experiment also highlights the effect of tool velocity on precipitate fraction evolution. For the highest velocity $\left(v=8 \mathrm{~mm} \cdot \mathrm{s}^{-1}\right)$, the peak temperature decreases rapidly and consequently the precipitate dissolution process is suppressed similarly. For the lower velocity, the time to develop precipitates dissolution is larger leading to a lower amount of residual precipitates. A comparison between precipitate volume fraction based on SAXS measurement and numerical modelling for the $\eta$ ' precipitate is proposed on Fig. 43 b) from the weld centre to the border of the tool. According to the authors, even if some differences may exist, a reasonable agreement is observed between experiment and simulation considering the complex phenomena involved. In addition, both results show a general tendency of precipitate fraction increase with lateral distance. At low distance, precipitate may dissolve and reprecipitate as large $\eta$ particles on grain boundaries. At large distance, reprecipitation of fine particle may form after cooling.

(a)

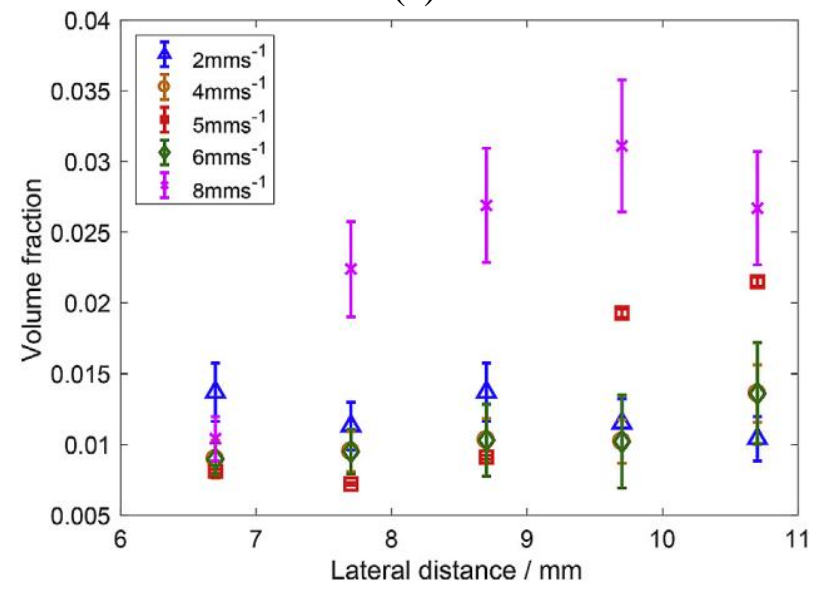

(b)

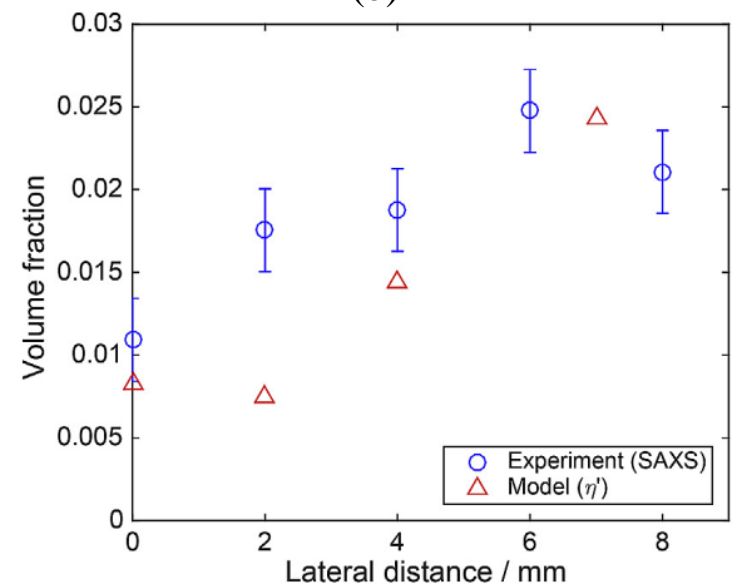

Fig. 43: (a) Volume fraction in HAZ for various welding speeds and lateral distances from the weld centreline. A lateral scan perpendicular to the tool direction (red line on Fig. 42) has been developed. (b) Comparison between model prediction (Kamp PSD model (Kamp et al. 2006, Kamp et al. 2007) ) and SAXS measurement on the final volume fraction of precipitates during welding (Dos Santos et al. 2018). 
This FlexiStir experimental system dedicated to FSW experiments combined with small angle X-ray scattering (SAXS) experiments gives the unique opportunity to track precipitate evolution during stirring process on aluminium alloys. However, as considered by Dos Santos et al. (2018), correct interpretation of experiments is only possible when these observations are regarded with results of PSD modelling. Such comparison may also highlight the contribution of each precipitate class on general precipitation process evolution. This equipment and the associated instrumentation demonstrate the possibilities provided by the more recent technical developments in material analysis. This system coupled with the SAXS measurements develops micro-scale analysis and gives access to data on material evolution until now inaccessible. This experiment is an innovative approach developed as an answer to researcher's need to follow microstructural evolution in FSW process and have direct estimation of precipitate distribution. Such experiment should be promoted to provide academic and industrial partners access to material evolution in FSW. More generally, SAXS measurements as developed by Dos Santos et al. (2018) are of great interest to estimate precipitate size evolutions however based on prior estimation of their size distribution function to develop fitting of the scattering curve. These observations are also of prime interest when applied on the new aluminium alloys based on the $\mathrm{Al}-\mathrm{Cu}-\mathrm{Li}$ system and recently proposed for future applications in aerospace industries. As an example, Steuwer et al. (2011) developed a wide range of analysis techniques to investigate the microstructural zones observed on AA2199 after welding, including SAXS, TEM, X-ray diffraction and neutron diffraction. Especially, the lack of W-shaped in hardness profile is explained by authors. More recently, De Geuser et al. (2014) investigated heterogeneous precipitation on AA2050-T8 grade after FSW and developed mapping by SAXS to estimate size and fraction of $T_{1}$ precipitates and GPB zones formed at ambient temperature. Both analyses are helpful in order to master and control hardening precipitates development and associated mechanical properties on these promising alloys. Consequently, these SAXS experimental developments should provide researchers access to direct or indirect observations of precipitate evolutions. This type of experiment gives possibilities to compare and validate models in literature also considering various affected domains. In the near future, we may recommend continuing the progress in such SAXS observations to improve, calibrate and validate PSD models.

\section{IV.2. Grain evolution modelling}

The previous chapter demonstrates that grain size evolutions in FSW are predominantly due to the Dynamic recrystallization (DRX) mechanism in the stir zone (SZ). DRX can be attributed to various mechanisms (DDRX, GDRX, CDRX). Consequently a large set of models and numerical approaches are available in literature depending from the choice and opinion of their authors on the origin of recrystallization mechanisms in SZ. However a summary of the mechanisms reported in literature can be proposed which will also highlight the current limitations of the associated models. 


\section{a. Recrystallization mechanisms}

Literature reports different simulations based on empirical models of DRX mechanisms (Table 3) as precisely detailed in previous sections. Grujicic et al. (2015) and Zhang et al. (2016) used Monte-Carlo models. Cellular automaton models were proposed by Buffa et al. (2007), Saluja et al. (2012) or Valvi et al. (2016). Semi-empirical models were also applied as detailed by Robson and Campbell (2010) in GDRX mechanisms. Physically-based models were similarly developed using the approach initially proposed by Gourdet and Montheillet (2003) (GM) and subsequently applied by Jacquin (2009) in FSW processes as GM-CDRX models. The main limitation of these models is their extreme difficulty to estimate the deformation path endured by the stirred material in the welding zone and proposed valuable estimation. Indeed accurate measurements of these deformations in FSW process are still unreachable even if some experimental developments are proposed to achieve comparable thermomechanical treatment as proposed by Masaki et al. (2008). Consequently, the DDRX, GDRX, and CDRX models are based on estimated or computed strain field requiring complex setting up of various parameters which sometime leads to important time-consuming calibration tests.

\section{- $\quad D D R X$}

The DDRX model has been applied by Hofmann and Vecchio (2007) as based on the approach originally proposed by Derby and Ashby (1987) however limited to the investigation of FSP and SFSP processes. This model is mainly based on the grain boundary migration rate. This rate is also deduced from the grain boundary mobility and the store work energy of the subgrain walls. This later expression is still approximated. Hofmann and Vecchio rightly point that the Derby and Ashby migration model has been widely validated for isothermal transformation and lead to underestimation of grain size in anisothermal transformation when large cooling rate are observed. However Friction stir processing leads to valuable estimations of grain size evolution as moderate cooling rate are observed $(30 \mathrm{~K} /$ $20 \mathrm{~s}$ ). This model depends also from the initial grain size from which grain growth proceeds. This latter value is still difficult to estimate and is provided from other modelling approaches. However this model is interesting for the prediction of small grains size expected in specific Friction Stirring process developed onto aluminium alloys.

\section{- $G D R X$}

The concept of the GDRX originated in Mac Queen (1988) research activities in 1980. The forming of new structure of grain is considered as a consequence of changes in grain geometry. The GDRX modelling is used by Robson and Campbell (2010) in the modelling of microstructure evolution in FSW. They consider that the grain boundary diameter is linked to 
the shear strain and they set up criteria on the sub-grain size to determine the onset of recrystallization. The sub-grain size is given by a Zener-Hollomon parameter. The main assumption of this model corresponds to the hypothesis that initial grains are pinched during the deformation until new grains appear.

\section{- $\quad C D R X$}

The principle of the GM model is to provide description of a polycrystalline structure evolution through the distribution of dislocation density at grain boundaries and sub-grain boundaries during deformation. The use of Gourdet-Montheillet CDRX model to predict grain size requires determining precisely the nucleation and disappearance of dislocations during the thermomechanical cycle. This point corresponds to the main difficulty to overcome to achieve valuable estimation of grain size evolution. Indeed, during the initial stage of strain, the dislocations multiply and interact leading to an increase in strain hardening. In the same time, the recovery mechanisms are enhanced by the temperature increase. Finally, both phenomena are balanced and the finale microstructure reaches an equilibrium state. The post dynamic grain growth during cooling could then be considered and provide a more precise estimation of final grain size.

\section{- Monte Carlo models}

Monte Carlo models were proposed and applied to simulate grain structure evolutions as detailed previously (Table 3). However, these models do not consider the mobility of dislocations or the crystal disorientation and its effect. These limitations correspond to one of their drawback. Indeed no consideration on specific grain orientation is applied onto grain energy expression and possible change of cell state. Considering previous developments as CDRX mechanism, this hypothesis may also lead to discussions on grain definition as a minimum value of grain disorientation is usually assumed at grain boundaries. Indeed, in this latter approach, sub-grain and grain are distinguished with their disorientation angle between adjacent crystals. However, in our opinion, the main difficulty encountered in such approaches is the time-dependence evolution and its estimation. Indeed, an analytical relation is previously established or afterward calibrated between simulation time, $t$, and number of Monte-Carlo step, $M C S$, to provide the number of steps required to reach a given time. This relation derived from empirically and theoretically expressions with some strong hypotheses. In addition, such relation is unable to apply when temperature field (i.e. non homogenous temperature) are observed on simulated pieces.

\section{b. Recrystallization models validation - Fields measurement limitation}

The present review reveals also several scientific and technological obstacles to overcome in order to validate models and calibrate unknown parameters. As mentioned previously, the first limitation corresponds to the lack of direct measurements to estimate material 
deformation during its complex and rapid transformation stage. This problem therefore prevents identification of thermo-mechanical phenomena and measurement of associated effects as strain field or temperature evolution in the pin neighbourhood. In addition direct measurement of microstructural evolutions (precipitation phase change and recrystallization) remains challenge. Most models are therefore based on very strong assumptions as to whether a particular phenomenon exists or not.

If the deformation takes place at a sufficiently high temperature in aluminium alloys, recovery processes operate simultaneously with the strain evolution. These processes are called "dynamic" recovery and "dynamic" recrystallization. Aluminium alloys are characterized by their high dynamic recovery capacity as detailed by McQueen and Evangelista (1988) at low to moderate deformations $(\bar{\varepsilon}<1)$. This property is linked to very high stacking fault energy (HSFE) closed to $170 \mathrm{~mJ} . \mathrm{m}^{-2}$, promoting the deflected sliding and climbing of dislocations. This phenomenon thus should normally prevent any DDRX phenomenon (nucleation and growth of new grains) even if some models corresponding to DDRX stages have been proposed in order to analyse grain growth mechanism as the model of Derby and Ashby and its derivatives (Table 3). However, it is no more obvious that the grain size refinement is governed by the mechanisms of CDRX than GDRX. Nevertheless, when we observe the DRX zone, the density of grain boundaries is substantially increased. The model based on the pinching of pre-existing grain boundaries is reasonably not sufficient to increase the grain boundary density. In addition the mechanisms of grain boundaries elongation or serration described by Mac Queen (2004) may not explain such an increase in wall surface density. The CDRX model, on the other hand, assumes that the grain boundary density can increase with strain hardening and deformation. Consequently, the main limitation in the CDRX model lies in the accuracy of the dislocation production estimation, the accuracy of the deformation field assessment and the accuracy in the computation of strain rate and temperature field. Numerous models predicting these fields in the stir zone are available in the literature. However it is extremely difficult to make accurate local measurements during FSW process to validate these models. As a consequence, all thermomechanical models are currently considered as only predictive and indirect validations are solely achieved.

It should be also pointed out that the CDRX models are still not sufficiently applied in FSW research works. One can find some scarce uses in $\mathrm{PhD}$ research activities and some uses in Buffa models. Researchers often prefer the growth nucleation models as the one proposed by Derby-Ashby (1987) (DDRX) and used by Hofmann and Vecchio (2007) even if aluminium alloys should be better modelled by CDRX approaches as high stacking fault energy material. However this uses is also explained by the lack of direct observation of recrystallization phenomena during FSW. Further investigations on CDRX and DDRX or their coexistence are required to provide more versatile or general model. Moreover, current recrystallization models are based on the assumption of pure metals and completely neglect the interactions of solutes or second phase particles even if they have some slowing down effects on grain boundary migration as demonstrated by Robson and Campbell (2010). 


\section{c. Scale and coupling limitation}

Another obstacle in the development of reliable recrystallization model is the scale changes. Indeed, as discussed previously, the microstructural modelling of FSW involves a large range of scales. Thermo-mechanical phenomena evolve from microscopic scales (dislocation, precipitation, grain) to macroscopic scales (hardness, thermal fields and flow lines). Homogenization methods developed to couple afterwards one scale to another are complex steps limiting thermo-mechanical and microstructural coupling models. Current researches propose to decouple the mechanical field computation from microstructural phenomena simulation even if complex interactions occur (Fig. 4). As an example, for the CDRX model, a mechanical simulation is firstly performed. As a second step, a particle is extracted and tracked along its flow line to follow its microstructural evolution. A continuous recrystallization model is then applied on the reconstructed thermo-mechanical history. Two types of methods are commonly used to determine thermo-mechanical fields.

On the one hand, authors such as Valvi et al. (2016) use models relying on simplified analytical field modelling. These analytical fields are much easier to implement than the numerical fields and require few computational resources. The results are almost immediately obtained and make possible to ignore the problems associated to scale change due to the general homogenization associated to these models. Nevertheless, analytical models are often approximate but sufficient to provide an order of magnitude on the results associated to investigated phenomena. In practice, these models add decoupling to the strategy as the authors separate the thermal and mechanical aspects. The displacement field in material is imposed from analytical development and the thermal field induced by plastic deformation is deduced. On the other hand, for numerical simulation, finite element methods are the most common. Finite element models are accurate and effective in solving thermo-mechanical problems with strong coupling. Nevertheless, these approaches are sensitive to the geometry of the problem. Moreover, the FSW process imposes numerous remeshing operations due to the high complexity of the strain fields which further penalize computing times. The main problem arising with these two models has to be pointed out. Indeed, both are exclusively based on empirical behaviour laws of power law type which are strongly dependent on the material hardening. Consequently, such approaches cannot be assumed as based on fully coupled material-thermo-mechanical constitutive law.

\section{d. Perspectives}

The previous points raised a current state of the art of recrystallization models in FSW processes also pointing their intrinsic limitations. Indeed the current strategies still show clear difficulties to propose relevant estimations of grain size evolution considering the complex thermo-mechanical treatment endured by materials during joining processes. Some perspectives are drawn hereafter to improve these recrystallization models and their 
application for future years.

\section{- $\quad$ Toth model}

An alternative to the GM model could be the Toth (T) CDRX model detailed by Toth et al. (2010) and based on crystal plasticity and grain fragmentation approach. This model is also able to predict grain size evolution and misorientation distribution, crystallographic texture as well as strain hardening in materials. This model considers lattice curvature developed in grains enduring deformation. The main assumption is that lattice rotation in individual grains is assumed as non-uniform. This rotation is affected and limited close to grain boundaries by the constraining effects of neighbouring grains. The lattice rotation is assumed as smaller close to the grain boundaries than in the middle part of grains. The grain is consequently shared into two areas corresponding respectively to zones affected and non-affected by grain boundaries. Thus, the differences in the amount of lattice rotation create a curvature within the crystallographic plane (Fig. 44).

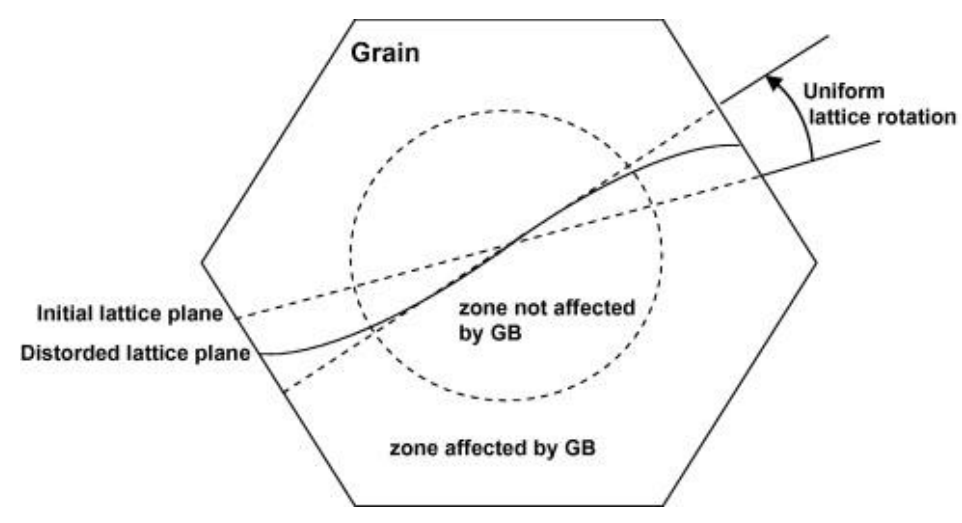

Fig. 44: Lattice curvature image showing the initial and distorted lattice plane (GB : Grain boundary) (Toth et al., 2010)

On this basis, the curvature variation is considered as equivalent to a geometrically necessary dislocation (GND) distribution. So that, when the strain is sufficiently large, the lattice curvature will be sufficient to create a new grain boundary. A scheme of fragmentation mechanism is then proposed and considered in the Taylor viscoplastic polycrystal model. The strain hardening modelling and by extension the misorientation distribution are based on the two-phase dislocation cell approach developed by Estrin et al. (1998). The GM- and TCDRX models are both based on a relevant distribution of dislocations generated by the hardening or dissolved by the recovering mechanisms. In the GM model, the new grains are globally homogenously distributed through the microstructure, while the new developed grains are mainly created close to grain boundaries in the T model. Unfortunately, the CDRX models dedicated to follow grain evolution in FSW on aluminium alloys are not yet widespread in literature. In many cases, the authors simply produce empirical descriptions of 
phenomena and apply empirical models that accurately reproduce observed decrease in grains size. These models are associated with well-defined operating conditions and are generally difficult to generalize to any type of experimental conditions: operating parameters, choice of alloy grade or thermal pre-treatments.

\section{- Towards multi-coupled and multiscale modelling.}

The simulation of the overall processes from microscale to macroscale will allow an optimisation of the final macro-manufactured product (i.e. choice of materials best suited for the production method). More efficient parts will be produced or reduced production costs will be obtained in order to promote the extension of FSW technologies in industries. Unlike the empirical laws currently in use, the physically based fully coupled thermomechanical and microstructural formulation will provide further improvements. We may cite the possibility to predict microstructural characteristics of parts, the possibility to prevent an unsuitable choice of materials or the possibility to optimise process conditions for a given service life specification. The achievement of this objective will undoubtedly pass in the near future by the use of crystal plasticity and homogenization law.

\section{- Develop measurement technics}

The improvement of FSW modelling will necessarily require the development of new experimental measurement protocols or facilities to avoid or optimize the tedious calibration steps and to identify precisely the transformation mechanisms. Some experimental developments have been recently presented in this way as the one proposed by Masaki et al. (2008) and dedicated to the investigation of thermo-mechanical treatments endured by Al 1050 aluminium grades in FSW processes with relevant conclusions on the range of deformations rate encountered by materials and associated microstructure.

\section{- Dissimilar welding}

In recent years, the scientific community seems particularly interested in the study of dissimilar welding as shown by Hamilton et al. (2019). Indeed, FSW process has the advantage to develop welding on alloys usually considered as non-weldable until now. An interesting development of such studies could be the analysis of DRX phenomena endured by materials during processes. The localization and kinetics of microstructure transformations in such welding conditions would be of clear interest. Some investigations may be developed in order to find the material where recrystallization first occurs as well as the type of DRX process considering properties associated to each material grade.

\section{- Toward future applications of FSW processes}

One of the interesting prospects for the evolution of FSW modelling could be to consider this process as a full-fledged thermo-mechanical treatment. Indeed, FSW is an efficient, fast 
and inexpensive technology that makes possible to obtain a homogeneous and stable refined microstructure. In this sense, this process could improve locally and easily the properties of a part through a controlled change of its microstructure. This change may be limited to specific areas, without affecting the whole piece. For example, the in-service resistance, corrosion or friction properties could be improved in a similar approach than the one proposed in SFSP process by Hofmann and Vecchio (2007). A localized doping with alloying elements could also enhance aluminium plates properties, based on heterogeneous welding techniques, and could retain all the advantages of FSW, including solid solution and no melting bath. In addition, this advantage can also be linked to the one recently highlighted in the application of FSW and related FSP techniques to metal matrix composites (MMC) in aluminium alloys. Indeed, Ju et al. (2017) investigated MMC made of uniformly distributed $\mathrm{TiB}_{2}$ particles in a fine grained structure of $\mathrm{Al}-\mathrm{Zn}-\mathrm{Mg}-\mathrm{Cu}$ alloy. When applying FSP on samples, reinforcement by in-situ TiB2 particles was achieved regarding end-use properties. Indeed, stirring processes solve the problems of cast defects occurrences encountered in MMC especially the ones related to particle clustering which have detrimental effects on solution treatments and age hardening steps. FSW and FSP processes promote a more uniform distribution of nanoparticles (e.g. $\mathrm{TiB}_{2}$ ) in the metal matrix even more when considering multi-pass method developed along the same line. The distribution of composite particles is enhanced by stirring thus limiting the formation of these cluster particles. Stable microstructures are also obtained as thermal treatments have limited consequences on grain size distribution. In addition, no abnormal grain is observed after heating as added particles have favourable pinning effect. Beneficial consequences on further diffusion process are consequently demonstrated by Ju et al. . Avettand-Fènoël and Simar (2016) have also developed a review demonstrating the advantages to apply FSW processes on MMC materials. Final microstructure obtained after joining process and material consequences were described. Final properties were also discussed regarding the behaviour of reinforcement induced by joining process. Challenges for future years were also highlighted regarding current expectations and industrial needs.

A combination of techniques based on FSW processes should also be investigated. For example, the coupling of FSW with additive manufacturing (AM) could completely re-texture microstructure of manufactured parts. Removal of porosities, refinement of texture or microstructure homogenization could be achieved with this approach. Some recent results have shown the benefit of FSW process and related processes on microstructural and mechanical properties of parts made by AM technology. Mukherjee et al. (2011) were among the first to identify the interest of FSP to enhance end-use properties of parts made by Direct Metal Deposition (DMD) methods. Indeed, this AM process is largely used in industries as repairing method however limited by some defects development such as porosities or cracks. Consequently, surface modifications induced by FSP may provide superior quality for parts as investigated on copper-nickel alloys. Mukherjee et al. have demonstrated that coupling DMD and FSP methods leads to porosities reduction, enhanced chemical homogenization and higher yield strength compared to the single DMD process. Similarly, Scherillo et al. (2017) have investigated the interest of FSW process to join parts made by Direct Metal Laser 
Sintering (DMLS) in order to develop large dimension pieces. The parent microstructure of DMLS parts has been initially studied on AlSi10Mg aluminium alloy. Recrystallization effect, grain refinement and changes in initial distribution of intermetallic phases induced later by FSW process have been investigated in the weld zones. Especially, initial microstructure characterized by successive deposited layers is replaced by homogeneous structure with fine grains. In addition, increase of the order of $10 \%$ in final microhardness is achieved after FSW in the SZ. Recently Du et al. (2018) have also highlighted the advantages of FSW process on the same AlSi10Mg alloy in order to join complex shapes as obtained in additive manufacturing processes showing the defects induced by insufficient heat input.

Rivera et al. (2018) report and investigate the Additive Friction Stir (AFS) process corresponding to a recent innovation in the field of AM also known as MELD. AFS is an innovative solid-state thermo-mechanical process combining FSW (no melting) and AM (freeform process) advantages as described in Fig. 45. During the process a solid state feedstock is added layer by layer to a substrate by stirring. The heat is generated by friction and deformation similarly to FSW process. The shoulder then plays the same role as in FSW. The process is able to produce complex parts with interesting metallurgical properties. The final microstructure is fully recrystallized with massive generation of equiaxed and refined grains. Exceptional properties are consequently obtained compared to parts manufactured by other AM processes where solidification stage may lead to porosities development. AFS can be used to repair, join or add secondary features. In addition AFS process would reduce production time and hence production costs considering the possibility to tailor final metallurgical state.

(a)

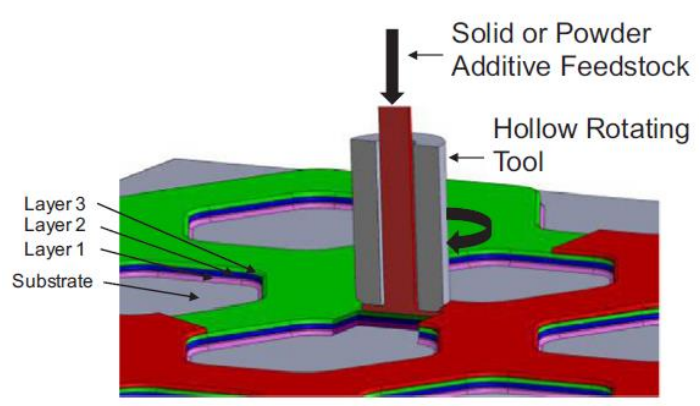

(b)

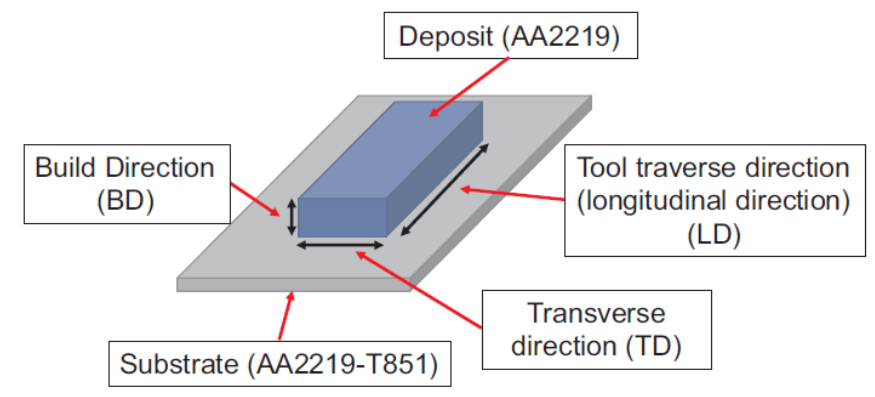

Fig. 45: (a) Scheme of the solid state material deposition (MELD) process. Solid feedstock material is extruded through a hollow tool. (b) Scheme of the as-deposited AA2219 showing the longitudinal, transverse, and build directions (Rivera et al. 2018). 


\section{Conclusion}

The modelling of microstructural evolution in aluminium alloys during FSW is required in order to investigate metallurgical changes endured by materials during joining process. These evolutions are observed at various scales and have large consequences on enduse properties of pieces with influences demonstrated on yield strength, fracture toughness, fatigue life or corrosion resistance. Therefore, understanding and mastering microstructure evolution is required to promote the use of FSW process as an alternative to other joining processes considering its clear advantages. Microstructure changes are observed at micro- and meso-scale when corresponding to both precipitate and grain size evolution mechanisms. At micro-scale, the dissolution, nucleation and growth of the small dispersed precipitates correspond to modification in present precipitate distributions inside SZ, HAZ and TMAZ domains. Nucleation of new intermetallic phases and complete dissolution of initial phases are observed. The modelling of precipitate dissolution was previously based in literature on the use of semi-analytical developments and master curves to track decrease in precipitate fraction at a given temperature. Experimental observations on the remaining precipitates during heat treatment were also required in a calibration stage. Recent models are mainly based on PSD approach where a class model mimics the size distribution of precipitates and its evolution as based on Myhr and Grong approach. Consequently, modelling of concurrent precipitates development in multicomponent and multiphase alloys is currently accessible. A clear advantage is provided by the complete description of precipitate distribution size with finer estimation of growing and dissolution kinetics. In addition, such model can be applied in any position of the welded domain in order to give estimation of final material state provided that time temperature evolution is accessible. An enhancement in the estimation of end-use mechanical properties considering contribution of each class is thus achieved by current models of literature.

At a larger scale all authors report the development of refined, equiaxed and homogeneous grains located in the stir zone. This area and its associated properties focus the interest of researchers. Grain size changes are mainly induced by dynamic recrystallization (DRX) mechanism. Modelling approaches are available in literature to track these evolutions, depending from the choice of authors in the DRX process when considering DDRX, GRX or CDRX approach. Nevertheless, even if several models dedicated to recrystallization mechanism are available in literature, validations are still limited and indirectly obtained when final microstructures are compared to simulations. Direct access to the stir zone and its continuous evolution are prevented. Therefore only in situ measurements of temperature fields are available to validate or invalidate the proposed models and their consequences on end-use properties.

In addition, influences of microstructural evolution are rarely considered in thermomechanical simulations of FSW process due to the difficulties to implement such complex coupled computations in a multi-scale approach. Hopefully, the increase in computer 
performances observed in recent years may help to propose thermo-mechanical and microstructural strong coupling in FSW modelling also considering decrease in investigated scales. In addition, thermodynamic coupling based on relevant database will provide similarly deep insight of precipitate evolution and phase fraction changes. We may see shortly such coupling between thermo-mechanical macro-scale resolution and fine micro-scale microstructural modelling. These numerical developments will undoubtedly provide relevant approaches to optimize at both scales process parameters to better control FSW processes and improve its development. Consequently, modelling of microstructural evolution is also part of the strategy required in order to disseminate FSW process in future years in industries as an alternative to other current joining processes. 


\section{References}

Aaron H.B., Fainstein D., Kotler G.R., 1970. Diffusion-Limited Phase Transformations : A comparison and Critical Evaluation of the Mathematical Approximations, Journal of Applied Physics 11, 4404-4410.

Abaqus Unified Fea, 2019. Dassault Systèmes, Simulia products, www.3ds.com/fr/produitset-services/simulia/produits/abaqus

Agelet de Saracibar C., López R., Chiumenti M., De Meester B., 2012. Material Characterization and FSW Process Optimization Using Neural Networks, ESMC 2012, 8th European Solid Mechanics Conference, Graz, Austria, 9-13 July 2012.

Agelet De Saracibar C., 2019. Challenges to be tackled in the computational modeling and numerical simulation of FSW processes, Metals 9, 573.

Amelinckx S., Dekeyser W., 1959. The Structure and Properties of Grain Boundaries, in: Seitz, F., Turnbull, D. (Eds.), Solid State Physics. Academic Press, 325-499.

Arora A., Zhang Z., De A., DebRoy T., 2009. Strains and strain rates during friction stir welding, Scripta Materialia 61, 863-866.

Aval H.J., Serajzadeh S., Kokabi A.H., 2011. Thermo-mechanical and microstructural issues in dissimilar friction stir welding of AA5086-AA6061, Journal of Materials Science 46, $3258-3268$.

Avettand-Fènoël M.-N., Simar A., 2016. A review about Friction Stir Welding of metal matrix composites. Materials Characterization 120, 1-17.

Bardel D., Perez M., Nelias D., Deschamps A., Hutchinson C.R., Maisonnette D., Chaise T., Garnier J., Bourlier F., 2014. Coupled precipitation and yield strength modelling for non-isothermal treatments of a 6061 aluminium alloy, Acta Materialia 62, 129-140.

Bitondo C., Prisco U., Squilace A., Buonadonna P., Dionoro G., 2010. Friction-stir welding of AA 2198 butt joints: mechanical characterization of the process and of the welds through DOE analysis. The International Journal of Advanced Manufacturing Technology, 53(5-8), 505-516.

Bjørneklett B.I., Grong Ø., Myhr O.R., Kluken A.O., 1999. A process model for the heataffected zone microstructure evolution in Al-Zn-Mg weldments, Metallurgical and Materials Transactions A, 30A, 2667-2677.

Blondeau R., 2013. Metallurgy and mechanics of welding: processes and industrial applications, John Wiley \& Sons (Eds).

Buffa G., Fratini L., Shivpuri R., 2007. CDRX modelling in friction stir welding of AA7075T6 aluminum alloy: Analytical approaches, Journal of Materials Processing Technology 191, 356-359. 
CALPHAD, Computer Coupling of Phase Diagrams and Thermochemistry, 2019, www.calphad.org

Carozzani T., Digonnet H., Gandin Ch-A., 2012. 3D CAFE modeling of grain structures: application to primary dendritic and secondary eutectic solidification, Modelling and Simulation in Materials Science and Engineering 20, 015010.

Castro-Fernáandez F.R., Sellars C.M., 1989. Relationship between room-temperature proof stress, dislocation density and subgrain size, Philosophical Magazine A, 60 (4), 487506.

Cazes R., 2003, Soudage par friction-malaxage, Réf. BM7746 v1, In Techniques de l'ingénieur (Eds).

Chen S., Guillemot G., Gandin Ch.-A., 2016. Three-dimensional cellular automaton-finite element modeling of solidification grain structures for arc-welding processes, Acta Materialia 115, 448-467.

Chen Y.C., Feng J.C., Liu H.J., 2009. Precipitate evolution in friction stir welding of 2219-T6 aluminum alloys. Materials Characterization 60, 476-481.

Chen Z., Cui S., 2008. On the forming mechanism of banded structures in aluminium alloy friction stir welds. Scripta Materialia, 58(5), 417-420.

Chovet C., Gourdet S., Montheillet F., 2000. Modelling the transition from discontinuous to continuous dynamic recrystallization with decreasing purity in aluminium, Materials Transactions, JIM 41, n 1, Special Issue on Ultra-High Purity Metals, 109-112.

Colegrove P., Painter M., Graham D., Miller T., 2000. 3 Dimensional Flow and Thermal Modeling of the Friction Stir Welding Process, Proceedings of the Second International Symposium on Friction Stir Welding, June 26-28, Gothenburg, Sweden.

Colegrove P.A., Shercliff H.R., 2004a. Development of Trivex friction stir welding tool - Part 1 - Two- dimensional flow modelling and experimental validation. Science and Technology of Welding and Joining, 9 (4), 345-351.

Colegrove P.A., Shercliff H.R., 2004b. Development of Trivex friction stir welding tool Part 2 - Three-dimensional flow modelling. Science and Technology of Welding and Joining, 9 (4), 352-361.

Colligan K., 1999. Material flow behaviour during friction welding of aluminium, Welding Research, Supplement to the Welding Journal, 78(7), 229-s - 237-s.

Colligan K.J., Mishra R.S., 2008. A conceptual model for the process variables related to heat generation in friction stir welding of aluminum, Scripta Materialia, 58(5), 327-331.

COMSOL Multiphysics Modeling Software, 2019, Software Product Suite, comsol.com CSFP, Center for Friction Stir, 2019. https://cfsphome.org/content/superliner-ogasawara 
Darras B.M., Khraisheh M.K., 2008. Analytical Modeling of Strain Rate Distribution During Friction Stir Processing. Journal of Materials Engineering and Performance 17, 168177.

Das S., 2010. Modeling mixed microstructures using a multi-level cellular automata finite element framework, Computational Materials Science 47 (3), 705-711.

DEFORM, Design Environment for Forming, 2019, Scientific Forming Technologies Corporation (SFTC), www.deform.com

De Pari L., Misiolek W.Z., 2008. Theoretical predictions and experimental verification of surface grain structure evolution for AA6061 during hot rolling, Acta Materialia, 56, 6174-6185.

Derby B., Ashby M.F., 1987. On dynamic recrystallization, Scripta Metallurgica 21, 879-884.

Dixit V., Mishra R.S., Lederich R.J., Talwar R., 2009. Influence of process parameters on microstructural evolution and mechanical properties in friction stirred Al-2024 (T3) alloy, Science and Technology of Welding and Joining 14 (4), 346-355.

Dmitriev A.I., Kolubaev E.A., Nikonov A.Y., Rubtsov V.E., Psakhie S.G., 2014. Study patterns of microstructure formation during friction stir welding, Proceedings of XLII International Summer School-Conference APM 2014, 10-16.

Doherty R.D., Hughes D.A., Humphreys F.J., Jonas J.J., Juul Jensen D., Kassner M.E., King W.E., McNelley T.R., McQueen H.J., Rollett A.D., 1997. Current issues in recrystallization: a review, Materials Science Engineering A 238, 219-274.

Dos Santos J.F., Staron P., Fischer T., Robson J.D., Kostka A., Colegrove P., Wang H., Hilgert J., Bergmann L., Hütsch L.L., Huber N., Schreyer A., 2018. Understanding precipitate evolution during friction stir welding of $\mathrm{Al}-\mathrm{Zn}-\mathrm{Mg}-\mathrm{Cu}$ alloy through in-situ measurement coupled with simulation, Acta Materialia 148, 163-172.

Du Z., Tan M.J., Chen H., Bi G., Chua C.K., 2018. Joining of 3D-printed AlSi10Mg by friction stir welding, Welding in the World, 62 (3), 675-682.

ENABLE project, 2019. European Union's Marie Skłodowska-Curie Actions Innovative Training Networks H2020-MSCA-ITN-2017, grant agreement N764979, www.enableproject.com.

ESI Sysweld, 2019. Welding, Assembly and Heat Treatment Predictive Simulation, ESI Group, www.esi-group.com/software-solutions/virtual-manufacturing/weldingassembly/esi-sysweld

Estrin Y., Tóth L.S., Molinari A., Bréchet Y., 1998. A dislocation-based model for all hardening stages in large strain deformation, Acta Materialia 46 (15), 5509-5522.

Feulvarch E., 2012. Modélisation numérique du procédé de soudage par friction-malaxage, Réf. BM7764 v1, In Techniques de l'ingénieur (Eds).

FLUENT, 2019. ANSYS Fluent software, www.ansys.com/fr-fr/products/fluids/ansys-fluent 
Fratini L., Buffa G., 2005. CDRX modelling in friction stir welding of aluminium alloys. International Journal of Machine Tools and Manufacture, 45(10), 1188-1194.

Fratini L., Buffa G., Palmeri D., 2009. Using a neural network for predicting the average grain size in friction stir welding processes, Computers and Structures 87 (17-18), 1166-1174.

Frigaard Ø., 1999. A process model for friction stir welding of age hardening aluminium alloys, Ph. D. Thesis, Norwegian University of Science and Technology, Trondheim, IME-Report, 1999-5.

Frigaard Ø., Grong Ø., Midling O.T., 2001. A Process Model for Friction Stir Welding of Age Hardening Aluminium Alloys, Metallurgical and Materials Transactions A 32, 1189-1200.

Gallais C., Denquin A., Bréchet Y., Lapasset G., 2008. Precipitation microstructures in an AA6056 aluminium alloy after friction stir welding : Characterisation and modelling, Materials Science and Engineering A 496, 77-89.

Gastebois S., 2015. Simulation numérique 3D du FSW à l'aide d'une formulation ALE, Ph.D. Thesis (in French), Mines ParisTech.

Fourment L., Gastebois S., Dubourg L., 2016. Calibration of 3D ALE finite element model from experiments on friction stir welding of lap joints, AIP Conference Proceedings $1769,100006$.

Genevois C., 2004. Genèse des microstructures lors du soudage par friction malaxage d'alliages d'aluminium de la série 2000 et 5000 et comportement mécanique résultant. Ph.D. Thesis (in French), Institut National Polytechnique de Grenoble.

Genevois C., Deschamps A., Denquin A., Doisneau-Cottignies B., 2005. Quantitative investigation of precipitation and mechanical behaviour for AA2024 friction stir welds, Acta Materialia 53 (8), 2447-2458.

De Geuser F., Malard B., Deschamps A., 2014. Microstructure mapping of a friction stir welded AA2050 Al-Li-Cu in the T8 state, Philosophical Magazine 94, 1451-1462.

Gholinia A., Humphreys F.J., Prangnell P.B., 2002. Production of ultra-fine grain microstructures in Al-Mg alloys by conventional rolling, Acta Materialia 50, 44614476.

Gibson B., Lammlein D., Prater T., Longhurst W., Cox C., Ballun M., Dharmaraj K., Cook G., Strauss A., 2014. Friction stir welding: Process, automation, and control, Journal of Manufacturing Processes 16 (1), 56-73.

Gopkalo O., Liu X., Long F., Booth M., Gerlich A.P., Diak B.J., 2019. Non-isothermal thermal cycle process model for predicting post-weld hardness in friction stir welding of dissimilar age-hardenable aluminum alloys, Materials Science and Engineering: A 754, 205-215. 
Gourdet S., Konopleva E.V., McQueen H.J., Montheillet F., 1996. Recrystallization during Hot Deformation of Aluminium, Materials Science Forum 217-222, 441-446.

Gourdet S., 1997. Etude des mécanismes de recristallisation au cours de la déformation à chaud de l'aluminium, Ph.D. Thesis (in French), Institut National Polytechnique de Grenoble.

Gourdet S., Montheillet F., 2002. Effects of dynamic grain boundary migration during the hot compression of high stacking fault energy metals, Acta Materialia 50 (11), 2801-2812.

Gourdet S., Montheillet F., 2003. A model of continuous dynamic recrystallization, Acta Materialia 51, 2685-2699.

Grujicic M., Ramaswami S., Snipes J.S., Avuthu V., Galgalikar R., Zhang Z., 2015. Prediction of the Grain-Microstructure Evolution Within a Friction Stir Welding (FSW) Joint via the Use of the Monte Carlo Simulation Method, Journal of Materials Engineering and Performance 24 (9), 3471-3486.

Guerdoux S., 2007. Numerical simulation of the friction stir welding process, Ph.D. Thesis, Ecole Nationale Supérieure des Mines de Paris.

Guillemot G., Gandin Ch.-A., 2017. An analytical model with interaction between species for growth and dissolution of precipitates, Acta Materialia 134, 375-393.

Hamilton C., Kopyściański M., Węglowska A., Dymek S., Pietras A., 2016. A Numerical Simulation for Dissimilar Aluminum Alloys Joined by Friction Stir Welding. Metallurgical and Materials Transactions A 47, 4519-4529.

Hamilton C., Kopyscianski M., Weglowska A., Pietras A., Dymek S., 2019. Modeling, microstructure, and mechanical properties of dissimilar 2017A and 5083 aluminum alloys friction stir welds, Proceedings of the Institution of Mechanical Engineers Part B Journal of Engineering Manufacture 233 (2), 553-564.

Hattel J.H., Schmidt H.N.B., Tutum C., 2009.Thermomechanical Modelling of Friction Stir Welding, Trends in Welding Research, June 1 - 6, 2008, Callaway Garden Resort, Pine Mountain, Georgia, USA, ASM International Eds.

Heinz A., Haszler A., Keidel C., Moldenhauer S., Benedictus R., Miller W.S., 2000. Recent development in aluminium alloys for aerospace applications, Materials Science and Engineering: A 280, 102-107.

Hines J.A., Vecchio K.S., 1997. Recrystallization kinetics within adiabatic shear bands, Acta Materialia 45, 635-649.

Hofmann D.C., Vecchio K.S., 2007. Thermal history analysis of friction stir processed and submerged friction stir processed aluminum, Materials Science and Engineering A, 465 (1-2), 165-175.

Huang K., Logé R.E., 2016. A review of dynamic recrystallization phenomena in metallic materials, Materials \& Design 111, 548-574. 
Humphreys F.J., Hatherly M., 2004. Recrystallization and Related Annealing Phenomena, Elsevier Ltd, Oxford.

Jacquin D., 2009. Modélisation de l'histoire thermomécanique des zones soudées en Friction Stir Welding: Application à la prévision des microstructures, Ph.D. Thesis (in French), Ecole des Mines de St Etienne (EMSE).

Jacquin D., De Meester B., Simar A., Deloison D., Montheillet F., Desrayaud C., 2011. A simple Eulerian thermomechanical modelling of friction stir welding. Journal of Materials Processing Technology, 211 (1), 57-65.

Jata K.V., Semiatin S.L., 2000. Continuous dynamic recrystallization during friction stir welding of high strength aluminum alloys, Scripta Materialia, 43 (8), 743-749.

JMatPro, Practical Software for Materials Properties, 2019, Sente Software Ltd., www.sentesoftware.co.uk

Ju X., Zhang F., Chen Z., Ji G., Wang M., Wu Y., Zhong S., Wang H., 2017. Microstructure of Multi-Pass Friction-Stir-Processed Al-Zn-Mg-Cu Alloys Reinforced by Nano-Sized TiB2 Particles and the Effect of T6 Heat Treatment, Metals 7, 530.

Kah P., Rajan R., Martikainen J., Suoranta R., 2015. Investigation of weld defects in frictionstir welding and fusion welding of aluminium alloys, International Journal of Mechanical and Materials Engineering, 10 (26).

Kalemba I., Dymek S., 2016. Microstructure and properties of friction stir welded aluminium alloys. Welding International 30, 38-42.

Kamp N., Sullivan A., Tomasi R., Robson J.D., 2006. Modelling of heterogeneous precipitate distribution evolution during friction stir welding process, Acta Materialia 54 (6), 2003 2014.

Kamp N., Sullivan A., Robson J.D., 2007. Modelling of friction stir welding of 7XXX aluminium alloys, Materials Science and Engineering A 466, 246-255.

Khalkhali A., Saranjam M.J., 2015. Finite element simulation of microstructure evolution during friction stir welding of automotive aluminum parts, International Journal of Automotive Engineering 5 (1), 932-938.

Krishnan K.N., 2002. On the formation of onion rings in friction stir welds, Materials Science and Engineering A 327, 246-251.

Kumar K., Kailas S., 2008. The role of friction stir welding tool on material flow and weld formation. Materials Science and Engineering A 485(1-2), 367-374.

Kumar R., Pancholi V., Bharti R.P., 2018. Material flow visualization and determination of strain rate during friction stir welding, Journal of Materials Processing Technology 255, 470-476.

Laasraoui A., Jonas J.J., 1991. Prediction of steel flow stresses at high temperatures and strain rates, Metallurgical Transactions A 22 (7), 1545-1558. 
Lammlein D.H., DeLapp D.R., Fleming P.A., Strauss A.M., Cook G.E., 2009. The application of shoulderless conical tools in friction stir welding: An experimental and theoretical study, Materials \& Design, 30 (10), 4012-4022.

LAMMPS Molecular Dynamics Simulator, 2019. https://lammps.sandia.gov

Legrand V., 2015a. Modélisation des processus de précipitation et prédictions mécaniques résultantes dans les alliages d'aluminium à durcissement structural - Application au soudage par Friction Malaxage (FSW) de tôles AA2024, Ph.D. Thesis (in French), Mines ParisTech.

Legrand V., Gastebois S., Guillemot G., Gandin Ch.-A., Fourment L., 2015b. Microstructural evolution during friction stir welding on AA2024 aluminium alloys - Application to the prediction of the mechanical properties, $11^{\text {th }}$ Int. Sem. Numerical Analysis of Weldability, Austria, 2015, Math. Model. of Weld Phen. 11, Eds. C. Sommitsch, N. Enziger and P. Mayr, Verlag der Technischen Universität Graz (2016) 497-513.

Lenard J.G., Pietrzyk M., Cser L., 1999. Mathematical and Physical Simulation of the Properties of Hot Rolled Products, $1^{\text {st }}$ Edition, Elsevier Science Ltd.

Li T., Gan W., Khurana S., 2006. Friction stir welding of L80 and X70 steels, Proceedings of the $6^{\text {th }}$ International FSW Symposium.

Li W., Zhang Z., Li J., Chao Y., 2012. Numerical analysis of joint temperature evolution during friction stir welding based on sticking contact. Journal of Materials Engineering and Performance 21(9), 1849-1856.

Li Y., Murr L.E., McClure J.C.J., 1999. Solid-state flow visualization in the friction-stir welding of $2024 \mathrm{Al}$ to $6061 \mathrm{Al}$, Scripta materialia 40 (9), 1041-1046.

Lomolino S., Tovo R., dos Santos J., 2005. On the fatigue behaviour and design curves of friction stir butt-welded Al alloys, International Journal of Fatigue 27(3), 305-316.

Lopez R., Ducoeur B., Chiumenti M., De Meester B., Agelet de Saracibar C., 2008. Modeling Precipitate Dissolution in Hardened Aluminium Alloys using Neural Networks, International Journal of Material Forming 1, Suppl 1, 1291 -1294.

Ma Y.E., Xia Z., Jiang R., Li W., 2013. Effect of welding parameters on mechanical and fatigue properties of friction stir welded 2198 T8 aluminium-lithium alloy joints, Engineering Fracture Mechanics 114, 1-11.

Magalhães A., 2016, Licentiate Thesis, Production Technology, Thermo-electric temperature measurements in friction stir welding - Towards feedback control of temperature, University West, Trollhättan, Sweden.

Mahoney M.W., Rhodes C.G., Flintoff J.G., Spurling R.A., Bingel W.H, 1998. Properties of friction-stir-welded 7075 T651 aluminium, Metallurgical and Materials Transactions APhysical Metallurgy and Materials Science 29 (7), 1955-1964. 
Masaki K., Sato Y.S., Maeda M., Kokawa H., 2008. Experimental simulation of recrystallized microstructure in friction stir welded Al alloy using a plane-strain compression test, Scripta Materialia 58 (5), 355-360.

McNelley T.R., Swaminathan S., Su J.Q., 2008. Recrystallization mechanisms during friction stir welding/processing of aluminum alloys, Scripta Materialia, 58 (5), 349-354.

McQueen H.J., Evangelista E., 1988. Substructures in aluminium from dynamic and static recovery, Czechoslovak Journal of Physics B 38 (4), 359-372.

McQueen H.J., 2004. Development of dynamic recrystallization theory, Materials Science and Engineering: A 387-389, 203-208.

Mishra R., Ma Z., 2005. Friction Stir Welding and Processing, Materials Science and Engineering: R: Reports 50 (1-2), 1-78.

Montheillet F., Le Coze J., 2002. Influence of Purity on the Dynamic Recrystallization of Metals and Alloys, $7^{\text {th }}$ Int Conf on Ultra-High Purity Materials (UHPM-00), Helsinki (Finland), Physica Status Solidi, Applied Research 189 (1), 51-58.

Mukherjee S., Ghosh A.K., 2011. Friction stir processing of direct metal deposited coppernickel 70/30, Materials Science and Engineering A 528, 3289-3294.

Myhr O.R., Grong Ø., 1991. Process modelling applied to 6082-T6 aluminium weldments - I. Reaction kinetics, Acta Metallurgica et Materialia 39 (11), 2693-2702.

Myhr O.R., Grong Ø., Klokkehaug S., Fjoer H.G., Kluken A.O., 1997. Process model for welding of Al-Mg-Si extrusions Part 1: Precipitate stability, Science and Technology of Welding and Joining, 2 (6), 245-253.

Myhr O.R., Klokkehaug S., Grong Ø., Fjær H.G., Kluken A.O., 1998. Modeling of Microstructure Evolution, Residual Stresses and Distortions in 6082-T6 Aluminum Weldments, Welding Journal 77 (6), 286s-292s.

Myhr O.R., Grong Ø., 2000. Modelling of non-isothermal transformations in alloys containing a particle distribution, Acta Materialia 48, 1605-1615.

Nandan R., DebRoy T., Bhadeshia H. K. D. H., 2008. Recent advances in friction-stir welding - Process, weldment structure and properties, Progress in Materials Science 53 (6), 980-1023.

NASA, 2019, Orion Spacecraft, https://www.nasa.gov/feature/power-up-system-tests-prepareorion-for-deep-space-exploration

Nicolas M., Deschamps A., 2003. Characterisation and modelling of precipitate evolution in an $\mathrm{Al}-\mathrm{Zn}-\mathrm{Mg}$ alloy during non-isothermal heat treatments, Acta Materialia 51, 607794.

Nikonov A.Y., Dmitriev A.I., Konovalenko I.S., Kolubaev E.A., Astafurov S.V., Psakhie S.G., 2015. Features of interface formation in crystallites under mechanically activated diffusion. A molecular dynamics study, XIII International Conference on 
Computational Plasticity: Fundamentals and Applications COMPLAS 2015, Spain, 982-991.

Nikonov A.Y., Konovalenko I.S., Dmitriev A.I., 2016. Molecular dynamics study of lattice rearrangement under mechanically activated diffusion, Physical Mesomechanics 19 (1), $77-85$.

Patel V., Li W., Wang G., Wang F., Vairis A., Niu P., 2019. Friction Stir Welding of Dissimilar Aluminum Alloy Combinations: State-of-the-Art. Metals 9, 270.

Perez M., Dumont M., Acevedo-Reyes D., 2008. Implementation of classical nucleation and growth theories for precipitation, Acta Materialia 56 (9), 2119-2132.

Pineau A., Guillemot G., Tourret D., Karma A., Gandin Ch.-A., 2018. Growth competition between columnar dendritic grains - Cellular automaton versus phase field modeling, Acta Materialia 155, 286-301.

Potts R., 1952. Some generalized order-disorder transformations. Mathematical Proceedings of the Cambridge Philosophical Society, 48 (1), 106-109.

Prangnell P.B., Heason C.P., 2005. Grain structure formation during friction stir welding observed by the 'stop action technique', Acta Materialia 53 (11), 3179-3192

Rambabu G., Balaji Naik D., Venkata Rao C.H., Srinivasa Rao K., Madhusudan Reddy G., 2015. Optimization of friction stir welding parameters for improved corrosion resistance of AA2219 aluminum alloy joints, Defence Technology 11 (4), 330-337

Rambabu P., Eswara Prasad N., Kutumbarao V.V., Wanhill R.J.H., 2017. Aluminium Alloys for Aerospace Applications (Chap. 2), in. Aerospace Materials and Material Technologies, Indian Institute of Metals Series, Prasad N. Eswara, Wanhill R. J. H. (Eds), Springer Singapore.

Reynolds A.P., Lockwood W.D., Seidel T.U., 2000a. Processing-Property Correlation in Friction Stir Welds, Materials Science Forum, 331-337, 1719-1724.

Reynolds A.P., 2000b. Visualisation of material flow in autogenous friction stir welds, Science and Technology of Welding and Joining, 5 (2), 120-124.

Richards D.G., Prangnell P.B., Withers P.J., Williams S.W., Wescott A., Olivier E.C., 2006. FE Modelling of Mechanical Tensioning for Controlling Residual Stresses in Friction Stir Welds, Materials Science Forum 539-543, 4025-4030.

Rivera O.G., Allison P.G., Brewer L.N., Rodriguez O.L., Jordon J.B., Liu T., Whittington W.R., Martens R.L., McClelland Z., Mason C.J.T., Garcia L., Su J.Q., Hardwick N., 2018. Influence of texture and grain refinement on the mechanical behavior of AA2219 fabricated by high shear solid state material deposition, Materials Science \& Engineering A 724, 547-558.

Robson J.D., Campbell L., 2010. Model for grain evolution during friction stir welding of aluminium alloys, Science and Technology of Welding and Joining 15 (2), 171-176. 
Rougier L., Jacot A., Gandin Ch-A., Di Napoli P., Théry P.-Y., Ponsen D., Jaquet V., 2013. Numerical simulation of precipitation in multicomponent Ni-base alloys, Acta Materialia 61 (17), 6396-6405.

Russell K.C., 1970. in Phase Transformations, H.I. Aaronson (Ed.), American Society for Metals, Metals Park, OH, 219-64

Sahu P.K., Pal S., 2017. Mechanical properties of dissimilar thickness aluminium alloy weld by single/double pass FSW, Journal of Materials Processing Technology 243, 442-455.

Saluja R.S., Ganesh Narayanan R., Das S., 2012. Cellular automata finite element (CAFE) model to predict the forming of friction stir welded blanks, Computational Materials Science 58, 87-100.

Santiago D., Urquiza S., Lombera G., Vedia L.D., 2009. 3D Modelling of Material Flow and Temperature in Friction Stir Welding, Soldagem \& Inspeção, Brazilian Welding Association (ABS) 14(3), 248-256.

Sauvage X., Dédé A., Muñoz A.C., Huneau B., 2008. Precipitate stability and recrystallisation in the weld nuggets of friction stir welded $\mathrm{Al}-\mathrm{Mg}-\mathrm{Si}$ and $\mathrm{Al}-\mathrm{Mg}-\mathrm{Sc}$ alloys, Materials Science and Engineering: A 491, 364-371.

Scherillo F., Astarita A., di Martino D., Contaldi V., di Matteo L., di Petta P., Casarin R., Squillace A., Langella A., 2017. On the microstructure analysis of FSW joints of Aluminium components made via Direct Metal Laser Sintering, Proceedings of the $20^{\text {th }}$ International ESAFORM Conference on Material Forming, Dublin City, Ireland, 26-28 April 2017.

Schmidt H., Hattel J., Wert J., 2004. An analytical model for the heat generation in friction stir welding, Modelling and Simulation in Materials Science and Engineering 12 (1), 143-157.

Schmidt H., Hattel J., 2005. A local model for the thermomechanical conditions in friction stir welding, Modelling and Simulation in Materials Science and Engineering, 13 (1), 77 93.

Schmidt H.N.B., Dickerson T.L., Hattel J.H., 2006. Material flow in butt friction stir welds in AA2024-T3, Acta Materialia 54 (4), 1199-1209.

Schmitz G.J., Prahl U., 2016. Handbook of Software Solutions for ICME, Wiley VCH Weinheim (DE).

Scopus, 2019. www.scopus.com

Seidel T.U., Reynolds A.P., 2001. Visualization of the material flow in AA2195 Friction-Stir Welds using a marker insert technique, Metallurgical and Materials Transactions A, 32 (11), 2879-2884. 
Serio L.M., Palumbo D., De Filippis L.A.C., Galietti U., Ludovico A.D., 2016. Effect of friction stir process parameters on the mechanical and thermal behavior of 5754-H111 aluminum plates. Materials 9, 122.

Serrière M., Gandin Ch.-A., Gautier E., Archambault P., Dehmas M., 2002. Modeling of precipitation coupled with thermodynamic calculations, $8^{\text {th }}$ International Conference on Aluminium Alloys (Cambridge, GB) 2-5 July 2002, Eds. P. J. Gregson, S. J. Harris, Materials Science Forum 396-402, Trans Tech Publications Ltd (Aedermannsdorf, CH) 747-752.

Shercliff H.R., Russell M.J., Taylor A., Dickerson T.L., 2005. Microstructural Modelling in Friction Stir Welding of 2000 Series Aluminium Alloys, Mécaniques et Industries 6, $25-35$.

Shojaeefard M.H., Akbari M., Khalkhali A., Asadi P., Parivar A.H., 2014. Optimization of microstructural and mechanical properties of friction stir welding using the cellular automaton and Taguchi method, Materials \& Design 64, 660-666.

Silva A.C.F., De Backer J., Bolmsjö G., 2017. Temperature measurements during friction stir welding. The International Journal of Advanced Manufacturing Technology 88, 28992908.

Silva-Magalhães A., Backer J.D., Martin J., Bolmsjö G., 2019. In-situ temperature measurement in friction stir welding of thick section aluminium alloys. Journal of Manufacturing Processes 39, 12-17.

Simar A., Bréchet Y., De Meester B., Denquin A., Pardoen T., 2007. Sequential modeling of local precipitation, strength and strain hardening in friction stir welds of an aluminium alloy 6005A-T6, Acta Materialia 55 (18), 6133-6143.

Simar A., Bréchet Y., De Meester B., Denquin A., Gallais C., Pardoen T., 2012. Integrated modeling of friction stir welding of 6xxx series Al alloys: Process, microstructure and properties. Progress in Materials Science 57 (1), 95-183.

Song M., Kovacevic R., 2003. Thermal modeling of friction stir welding in a moving coordinate system and its validation, International Journal of Machine Tools and Manufacture 43 (6), 605-615.

Steuwer A., Dumont M., Altenkirch J., Birosca S., Deschamps A., Prangnell P.B., Withers P.J., 2011. A combined approach to microstructure mapping of an Al-Li AA2199 friction stir weld, Acta Materialia 59 (8), 3002-3011.

Su H., Wu C.S., Pittner A., Rethmeier M., 2013. Simultaneous measurement of tool torque, traverse force and axial force in friction stir welding. Journal of Manufacturing Processes 15, 495-500. 
Sullivan A., Robson J.D., Shercliff H.R., McShane G., 2007. Process Modelling of Friction Stir Welding for Aerospace Aluminium Alloys, Proceedings of the Thermec 2006, Vancouver, Canada, published in Advanced Materials Research 15-17, 351-356.

Svoboda J., Fischer F.D., Fratzl P., Kozeschnik E., 2004. Modelling of kinetics in multicomponent multi-phase systems with spherical precipitates: I: Theory, Materials Science and Engineering: A 385 (1-2), 166-174.

TC_PRISMA, 2019. Software, Thermo-Calc Software company, Solna, Sweden, www.thermocalc.com/products-services/software/precipitation-module-(tc-prisma)

TCAL3 - TCS Al-based alloy thermodynamic database, 2014. Thermo-Calc Software company

Thermo-Calc, Thermo-Calc Software Company, 2019. Solna, Sweden, https://www.thermocalc.com/

Thomas W.M., Nicholas E.D., Needham J.C., Church M.G., Templesmith P., Dawes C.J., 1995. Friction Stir Butt Welding, International Patent No. PCT/GB92/02203, GB Patent No. 9125978.8, 1991, U.S. Patent No. 5,460, 317.

Threadgill P., 1997. Friction stir welds in aluminium alloys: Preliminary microstructural assessment, TWI Bulletin, March/April, Abington UK.

Toth L.S., Estrin Y., Lapovok R., Gu C., 2010. A model of grain fragmentation based on lattice curvature, Acta Materialia 58 (5), 1782-1794.

TWI, The Welding Institute, 2019. www.twi-global.com

Ullegaddi K., Murthy V., Harsha R.N., Manjunatha, 2017. Friction Stir Welding Tool Design and Their Effect on Welding of AA-6082 T6. Materials Today: Proceedings 4, 79627970.

Valvi S.R., Krishnan A., Das S., Narayanan R.G., 2016. Prediction of microstructural features and forming of friction stir welded sheets using cellular automata finite element (CAFE) approach, International Journal of Material Forming 9 (1), 115-129

VIA Inno, 2017, Veille innovation Aquitaine, patent database orbit, http://viainno.ubordeaux.fr

Vilaça P., Quintino L., dos Santos J.F., Zettler R., Sheikhi S., 2007. Quality assessment of friction stir welding joints via an analytical thermal model, iSTIR. Materials Science and Engineering: A, 445-446, 501-508.

Wagner R., Kampmann R., 1991. Homogeneous Second Phase Precipitation, in: Cahn R., Haasen P., Kramer E. (Eds.), Phase Transformation in Materials, Material Science and Technology, 5, VCH, Weinheim, Germany, (Chapter 4) 213-303.

Wan Z.Y., Zhang Z., Zhou X., 2017. Finite element modeling of grain growth by point tracking method in friction stir welding of AA6082-T6, The International Journal of Advanced Manufacturing Technology 90 (9-12), 3567-3574. 
Wang X., Wang K., Shen Y., Hu K., 2008. Comparison of fatigue property between friction stir and TIG welds, Journal of University of Science and Technology Beijing, Mineral, Metallurgy, Material 15 (3), 280-284.

Woo W., Balogh L., Ungár T., Choo H., Feng Z., 2008. Grain structure and dislocation density measurements in a friction-stir welded aluminium alloy using X-ray peak profile analysis, Materials Science and Engineering: A 498 (1-2), 308-313.

Yang Y., Kalya P., Landers R.G., Krishnamurthy K., 2008. Automatic gap detection in friction stir butt welding operations. International Journal of Machine Tools and Manufacture 48 (10), 1161-1169.

Yi Y., Fu X., Cui J.-D., Chen H., 2008. Prediction of grain size for large-sized aluminium alloy 7050 forging during hot forming, Journal of Central South University of Technology 15 (1), 1-5

Zeldovich J., 1943. On the theory of new phase formation, cavitation, Acta Physicochimica U.R.S.S. XVIII, 1-22

Zhang Z., Wu Q., Grujicic M., Wan Z.Y., 2016. Monte Carlo simulation of grain growth and welding zones in friction stir welding of AA6082-T6, Journal of Materials Science 51 (4), 1882-1895.

Zimmer S., Langlois L., Laye J., Bigot R., 2010. Experimental investigation of the influence of the FSW plunge processing parameters on the maximum generated force and torque. The International Journal of Advanced Manufacturing Technology 47, 201-215. 